\title{
Delay Analysis of Social Group Multicast-Aided Content Dissemination in Cellular System
}

\author{
Jie Hu, Member, IEEE, Lie-Liang Yang, Fellow, IEEE, and Lajos Hanzo, Fellow, IEEE
}

\begin{abstract}
Based on the common interest of mobile users (MUs) in a social group, the dissemination of content across the social group is studied as a powerful supplement to conventional cellular communication with the goal of improving the delay performance of the content dissemination process. The content popularity is modeled by a Zipf distribution to characterize the MUs' different interests in different contents. The factor of altruism (FA) terminology is introduced for quantifying the willingness of content owners to share their content. We model the dissemination process of a specific packet by a pure-birth-based Markov chain and evaluate the statistical properties of both the network's dissemination delay as well as of the individual user-delay. Compared to the conventional base station (BS)-aided multicast, our scheme is capable of reducing the average dissemination delay by about $56.5 \%$. Moreover, in contrast to the BS-aided multicast, increasing the number of MUs in the target social group is capable of reducing the average individual user-delay by $\mathbf{4 4 . 1 \%}$ relying on our scheme. Furthermore, our scheme is more suitable for disseminating a popular piece of content.
\end{abstract}

Index Terms-Content dissemination, content popularity, factor of altruism, pure-birth based Markov chain, delay analysis.

\section{INTRODUCTION}

\section{A. Background and Related Works}

A $\mathrm{S}$ a combination of social science and mobile networks, mobile social networks (MSNs) [1] are attracting an increasing attention across the research community. In the context of MSNs, mobile users (MUs) may form a social group in order to cooperatively disseminate the content of common interest. There are substantial contributions to the performance analysis of epidemic forwarding [2] in mobile ad hoc networks (MANETs). In the context of MANETs, a two-dimensional continuous time Markov chain (CTMC) was proposed in [3] for evaluating the performance of a heterogeneous MANETs. To a further advance, the authors of [4] derived a tight upper bound of the flooding time, which is defined as the number of timesteps required for broadcasting a message from a source node to

Manuscript received April 12, 2015; revised October 18, 2015 and February 10, 2016; accepted February 22, 2016. This work was supported by the RCUK's India-UK Advanced Technology Centre (IU-ATC), that of the EU's concerto project and of the China Scholarship Council (CSC). The work of L. Hanzo was supported by the European Research Council under its Advanced Fellow Award. The associate editor coordinating the review of this paper and approving it for publication was B. Liang.

J. Hu is with the School of Communication and Information Engineering, University of Electronic Science and Technology of China, Chengdu 611731, China (e-mail: hujienavy@outlook.com).

L.-L. Yang and L. Hanzo are with the School of Electronics and Computer Science, University of Southampton, Southampton SO17 1BJ, U.K. (e-mail: 1ly@ecs.soton.ac.uk; 1h@ecs.soton.ac.uk).

Color versions of one or more of the figures in this paper are available online at http://ieeexplore.ieee.org.

Digital Object Identifier 10.1109/TCOMM.2016.2535330 all nodes. Furthermore, in [5] the end-to-end message delivery delay using an epidemic forwarding protocol was investigated theoretically in a composite twin-layer network, which includes a physical MANET and a virtual social network.

However, epidemic forwarding [6] is often criticised as being an end-to-end routing protocol, because it consumes substantial resources of the intermediate nodes, which might not be interested in the information to be relayed. However, if MUs can form a social group and request the content of common interest together, epidemic forwarding becomes an efficient way of cooperatively disseminating the content in the target social group $^{1}$. Content dissemination in purely distributed opportunistic networks was investigated in [7] and [8]. Epidemic forwarding aided content dissemination was invoked in [7], where the users share any content updates with others that they meet in order to improve the coverage quality and to increase the capacity. A socially-aware content placement algorithm was proposed in [8] for enhancing the opportunity of MUs to gain access to their contents of interest.

Some researches focused on a hybrid content dissemination approach. In [9] and [10], the authors investigated how the content providers and network operators can interact for the sake of efficiently distributing the contents with the aid of a coalition game. At the time of writing, epidemic forwarding aided content dissemination is widely studied for the sake of offloading tele-traffic from cellular networks. In [11], the authors proposed a framework for initial content-receiver selection in order to disseminate the content of common interest to as many subscribers as possible before interest in the content subsides. In [12], where MUs were categorised into "helpers" and "subscribers", several algorithms were designed for solving the optimisation problem of offloading multiple types of contents from the cellular networks.

The above-mentioned contributions [2]-[12] focused their attention on user-encounter-based MANETs or 'large-scale MSNs', where the mobile nodes are sparsely distributed across a large area. Typically a rudimentary physical layer model is assumed, namely that if a pair of nodes enter each other's transmission range, the packet can be successfully delivered from the source to the target. Hence, the delivery delay is dominated by the inter-contact duration ${ }^{2}$ of mobile nodes [15], rather than by the wireless signal propagation. Due to

\footnotetext{
${ }^{1}$ Other MUs that do not belong to the target social group are not relied upon for assisting the content dissemination process.

${ }^{2}$ In these treatises, the inter-contact duration of MUs is commonly assumed to obey an exponential distribution, which is demonstrated in [13] with the aid of artificial or synthetic mobility models and in [14] by realistic measured mobility traces.
} 
the underlying long inter-contact duration of the MUs, this user-encounter-based content dissemination is only capable of delivering delay-tolerant services in a large-scale area. As a result, the contributions of [2]-[12] belong to the category of delay-tolerant networks (DTNs). However, typically idealised simplifying assumptions are used in the literature of the DTN paradigm:

- The commonly assumed simplified physical layer model ignores the impact of transmit power, of the path-loss and of the multipath fading, etc.

- The cooperative user-encounter based content dissemination in DTNs is not suitable for delivering delay-sensitive services.

\section{B. Motivations and Contributions}

The conventional method of disseminating the delaysensitive content of common interest relies on BS-aided multicast, where the BS is the sole transmitter. Since the BS-aided multicast has to guarantee the quality of service (QoS) at every content requester, the capacity of multicast channels is predetermined by the worst channel amongst those connecting the BS to the content requesters. In this case, due to the time-variant nature of wireless channels, when the BS multicasts a packet, some MUs may receive it earlier than their less fortunate counterparts. Then, the successful receivers have to remain silent, because the BS would not multicast the second packet, before all the MUs successfully receive the current one.

In high-user-density scenarios, the MUs often share common interest in delay-sensitive content. For instance, the crowd participating in the inauguration of the new Pope share common interest in close-up video-clips of the Pope on the podium. Similarly, supporters in a football stadium share common interest in video-clips of a spectacular goal from different angles or in the score updates from another stadium, as exemplified by Fig. 1. However, the conventional BS-aided multicast is an inefficient technique of disseminating the delay-sensitive content of common interest in these typical densely populated scenarios. The reason for this is two-fold:

- As the content requesters' density increases, the worst channel amongst those connecting the BS and the content requesters becomes even worse, which results in excessive dissemination delay [16].

- Since the dissemination delay is increased, the BS is engaged in multicasting for a longer period, which further delays all other services.

If local MUs form a social group for requesting the content of common interest from the BS together, local communications amongst MUs can be exploited for cooperatively multicasting the packets from the packet owners to the hitherto unserved MUs in the target social group ${ }^{3}$. The potential performance gain of this social group multicast aided content dissemination over the conventional BS-aided multicast arises from the following two benefits:

\footnotetext{
${ }^{3}$ A similar methodology of improving BS-aided multicast was also advocated in [17], which was mainly focused on the selection of the initial receivers However, the authors of [17] have not analysed the content dissemination stage.
}
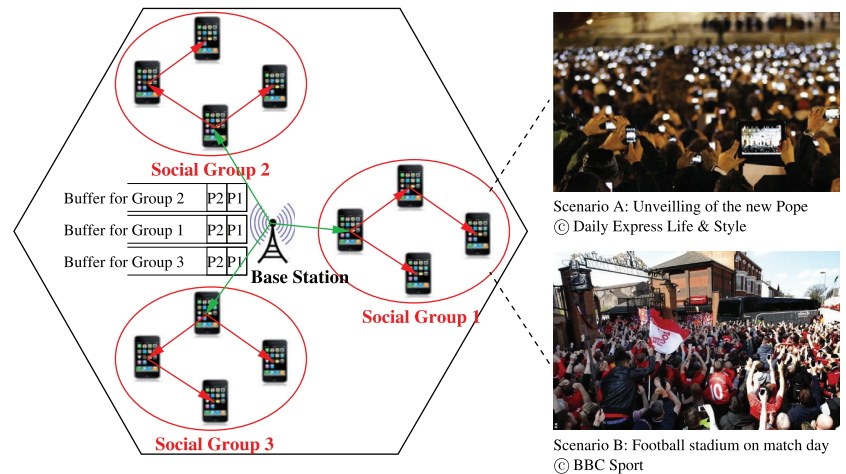

Fig. 1. Social group multicast aided content dissemination in cellular systems.

- Relying on the cooperative multicast of the multiple 134 packet owners results in rich cooperative diversity gains, 135 which in turn improves the packet delivery performance. 136

- Activating direct transmissions amongst the MUs is capa- 137 ble of reducing the distance between a transmitter and 138 receiver pair, which in turn reduces the path-loss-induced 139 channel attenuation between them.

Furthermore, since we offload the content dissemination task 141 from the BS-aided multicast to the local communications 142 amongst the social group members, the BS becomes capable of 143 satisfying other communication demands, which consequently 144 improves the efficiency of the BS's exploitation.

The size of the area covered by a social group should be carefully designed for different scenarios. If the area is as large as a macro-cell, cooperative user-encounter based communication amongst MUs is only suitable for disseminating delay-tolerant information, as we argued at the end of Section I-A. The best option for disseminating delay-sensitive information across a large area is that of classic BS-aided multicast. By contrast, if the area is relatively small, such as a circular area with a radius shorter than a hundred meters, which is comparable to the default transmission range of a $\mathrm{MU}^{4}$, communication efficiency between a transmitter and receiver pair is dominated by the wireless signal propagation properties, rather than by their inter-contact duration. Hence, social group aided cooperative multicast is capable of significantly reducing the delay of the conventional BS-aided multicast, as we emphasized at the beginning of Section I-B. This scenario is termed as a "small-scale MSN" [15], where the channel attenuation factors dominate the associated delay characteristics [19]. Against this background, our novel contributions are as follows:

- A hybrid content dissemination approach is proposed, which relies both on BS-aided multicast [20] and on social group multicast aided content dissemination. This process is modelled by a pure-birth based Markov chain $(P B M C)$. Various factors that might affect the performance of the content dissemination are accounted for, including the path-loss-induced channel attenuation, the multipath fading and the users' altruistic versus selfish behaviours, which distinguishes our work from the existing literature of DTNs.

${ }^{4} \mathrm{New}$ Wi-Fi protocols, such as $802.11 \mathrm{n} / \mathrm{ac}$ [18], are capable of supporting a transmission range of hundreds of meters. 
- We model the popularity of different pieces of contents by a Zipf distribution, which affects the specific formation of a social group and hence influences the dissemination process of the content of common interest across the target social group.

- Considering a specific packet of the content of common interest, we analyse the statistical properties of the dissemination delay, which is the time from the BS's instant of multicasting a packet until all the MUs in the target social group receive this packet. We also analyse the individual user-delay, which is the time spanning from the BS multicasting a packet until a specific MU receives this packet.

- The advantages of our social group multicast aided content dissemination scheme over the conventional BSaided multicast are demonstrated by the mobility traces extracted from a realistic subway station scenario.

Note that improving the network infrastructure in high-userdensity areas can certainly enhance the general communication experience of MUs, when supporting phone calls, texts, emails and basic data services. However, it may constitute an inefficient technique of disseminating the content of common interest. It may also be an unwise investment for the network operators, since people often temporarily get together for attending social events. Hence, improving the infrastructure capacity may be wasteful. By contrast, our social group multicast scheme constitutes a more economical and flexible solution for disseminating the content of common interest amongst the social group members, which is based on direct communications between the social group members. We will demonstrate that our social group multicast aided scheme outperforms the BS-aided multicast in terms of disseminating the popular content of common interest in high-user-density areas.

The rest of the paper is organized as follows. In Section II, our system model is introduced. In Section III, we analyse the delay metrics. Furthermore, the exact closed-form formulas are derived for two special cases in Section IV. Our numerical results are provided in Section V. Finally, we conclude in Section VI.

\section{SySTEM OVERVIEW}

Similar to the BS-controlled device-to-device communication services of the LTE network [21], our system operates by obeying a centralised-control regime combined with a decentralised-transmission paradigm ${ }^{5}$, where the BS acts as a centralised controller in order to support the functions of synchronisation ${ }^{6}$, of social group formation as well as of coordination and resource allocation for multiple content owners etc. By contrast, the information transmission is carried out by direct communications between a transmitter and receiver pair.

\footnotetext{
${ }^{5}$ This paradigm has been considered as a part of the forthcoming ' $5 \mathrm{G}$ ' regime, known as the 'LTE-Assisted Wi-Fi Direct' technique [22], where the control signalling exchange is carried out by the LTE-based BS, while the information transmission is realised by the Wi-Fi-based direct communication between a transmitter and receiver pair.

${ }^{6}$ Since the MUs in the cellular system rely on regular control signalling exchange with associated BSs, they can readily synchronise with associated BSs and hence also with each other.
}

TABLE I

The Request Probabilities of $\mathcal{M}=10$ Ranked Popular Contents FOR BOTH $\alpha=0.56$ [24] AND $\alpha=1.0$ [23]

\begin{tabular}{|c|c|c|c|c|c|}
\hline Content & $\mathcal{C}_{1}$ & $\mathcal{C}_{2}$ & $\mathcal{C}_{3}$ & $\mathcal{C}_{4}$ & $\mathcal{C}_{5}$ \\
\hline$\alpha=0.56$ & $21.4 \%$ & $14.6 \%$ & $11.6 \%$ & $9.9 \%$ & $8.7 \%$ \\
\hline$\alpha=1.0$ & $34.1 \%$ & $17.1 \%$ & $11.4 \%$ & $8.5 \%$ & $6.8 \%$ \\
\hline \hline Content & $\mathcal{C}_{6}$ & $\mathcal{C}_{7}$ & $\mathcal{C}_{8}$ & $\mathcal{C}_{9}$ & $\mathcal{C}_{1} 0$ \\
\hline$\alpha=0.56$ & $7.9 \%$ & $7.2 \%$ & $6.7 \%$ & $6.3 \%$ & $6.0 \%$ \\
\hline$\alpha=1.0$ & $5.7 \%$ & $4.9 \%$ & $4.3 \%$ & $3.8 \%$ & $3.4 \%$ \\
\hline
\end{tabular}

\section{A. Content Popularity and Social Group Formation}

The interest of a MU in a specific piece of content $\mathcal{C}_{i}$ may 225 be modelled by the probability $\operatorname{Pr}\left(\mathcal{C}_{i}\right)$ of this $\mathrm{MU}$ requesting $\mathcal{C}_{i} 226$ from the BS. Having a higher request probability $\operatorname{Pr}\left(\mathcal{C}_{i}\right)$ indi- 227 cates that the MU is more interested in the content $\mathcal{C}_{i}$. The 228 statistical analysis of the realistic video viewing behaviours 229 exhibited by YouTube users revealed that a small fraction of 230 popular contents attract the interest of a large fraction of users 231 [23], [24]. Furthermore, the request probabilities of a set of 232 ranked contents, say $\left\{\mathcal{C}_{i} \mid i=1, \ldots, \mathcal{N}\right\}$, may be modelled by 233 a Zipf distribution [25], [26]. Here $\mathcal{M}$ is the number of contents 234 studied and the subscript $i$ represents the particular position 235 of $\mathcal{C}_{i}$ in the popularity list. A smaller integer subscript $i$ indi- 236 cates that the content is more popular and hence it is likely to 237 be requested more frequently. Therefore, the probability of the 238 piece of content $\mathcal{C}_{i}$ being requested is expressed as

$$
\operatorname{Pr}\left(\mathfrak{C}_{i}\right)=\frac{\frac{1}{i^{\alpha}}}{\sum_{j=1}^{\mathcal{M}} \frac{1}{j^{\alpha}}},
$$

where $\alpha$ is a predefined exponent. Having a higher value of 240 $\alpha$ results in more intense interests in the top-ranked pieces of 241 contents, as shown in TABLE I.

Assuming that we have $\mathcal{N}$ MUs within the area studied, these 243 MUs independently request one piece of contents from the set 244 $\left\{\mathcal{C}_{i} \mid i=1, \ldots, \mathcal{M}\right\}$ with the corresponding probability defined 245 in (1). The MUs requesting the same content $\mathcal{C}_{i}$ form a social 246 group $\mathcal{G}_{i}$ in order to cooperatively disseminate the content of 247 common interest across the social group. Hence, the size of 248 the social group $\mathcal{G}_{i}$ requesting the same content $\mathcal{C}_{i}$ obeys a 249 Binomial distribution, which is denoted as $\left|\mathcal{G}_{i}\right| \sim B\left[\mathcal{N}, \operatorname{Pr}\left(\mathcal{C}_{i}\right)\right] .250$ In order to exclude the case of $|\mathcal{G}|=0$, we adjust the probability 251 mass function (pmf) of $\left|\mathcal{G}_{i}\right|$, which is expressed as

$$
\operatorname{Pr}\left(\left|\mathcal{G}_{i}\right|=N\right)=\frac{\left(\begin{array}{l}
\mathcal{N} \\
N
\end{array}\right)\left[\operatorname{Pr}\left(\mathcal{C}_{i}\right)\right]^{N}\left[1-\operatorname{Pr}\left(\mathcal{C}_{i}\right)\right]^{\mathcal{N}-N}}{1-\left[1-\operatorname{Pr}\left(\mathcal{C}_{i}\right)\right]^{\mathcal{N}}} .
$$

where $N$ is the specific size of the social group $\mathcal{G}_{i}$. As a result, 253 the average $\overline{\mathcal{P}}\left(\mathcal{C}_{i}\right)$ of a specific delay metric associated with dis- 254 seminating the content $\mathcal{C}_{i}$ across the social group $\mathcal{G}_{i}$, whose size 255 is an adjusted-Binomially distributed random variable, can be 256 expressed as

$$
\overline{\mathcal{P}}\left(\mathfrak{C}_{i}\right)=\sum_{N=1}^{\mathcal{N}} \mathcal{P}\left(\left|\mathcal{G}_{i}\right|=N\right) \cdot \operatorname{Pr}\left(\left|\mathcal{G}_{i}\right|=N\right),
$$

\footnotetext{
${ }^{7}$ If no MUs requests the content, we do not have to study the content dissemination performance.
} 
where $\mathcal{P}\left(\left|\mathcal{G}_{i}\right|=N\right)$ is a delay metric, which is a function of the deterministic social group size $\left|\mathcal{G}_{i}\right|=N$. Given the social group size $N$, in Section III, we will derive various delay metrics that can replace $\mathcal{P}\left(\left|\mathcal{G}_{i}\right|=N\right)$ in (3) in order to evaluate the impact of content popularity on the content dissemination performance.

To sum up, we assume that $N$ MUs form a social group in order to request the content of common interest from a BS, as shown in Fig. 1. The formation of a social group depends on the following conditions:

- MUs share the same interest in a given piece of content;

- The content of common interest is of delay-sensitive nature;

- MUs roam in a bounded area having a relatively small size and they are geographically close to each other.

\section{B. Network Layer}

In order to disseminate the content of common interest across a social group, the BS creates a specific queue for buffering all the packets of the requested content and prepares for disseminating these packets one by one, as described below.

Firstly, the BSs are employed for repeatedly multicasting the packet currently at the head of the buffer, until at least one of the MUs in the target social group successfully receives it. Then, this packet is cooperatively disseminated across the social group using multicast techniques.

During the dissemination process, after successfully receiving the packet, the packet owners (POs) may make their decisions independently as to whether they would or would not forward the packet during the following stage of the dissemination, as shown in Fig. 2. Once some POs decided to further forward the packet, they would repeatedly multicast it until at least one unserved MU in the target social group successfully receives it. Afterwards, the new POs join the original PO set. Both the new POs and the original POs make new packet forwarding decisions again for the subsequent stage of dissemination. The probability of a PO willing to forward the packet is denoted as $q(0 \leq q \leq 1)$, which is termed as the Factor of Altruism (FA). At a given instant, there might not be any POs willing to further forward the packet. As a result, the unserved MUs in the target social group have to receive the packet directly from the BS. Similarly, the BS repeatedly multicasts the packet until at least one unserved MU in the target social group receives it.

During the content dissemination process, similar to the conventional BS-aided multicast, the BS keeps a specific packet at the head of the buffer, until all the MUs in the target social group successfully receive it. Then the packet is dropped from the buffer and the BS is ready to disseminate the subsequent one.

\section{Physical (PHY) Layer}

In the PHY layer, the radio propagation between any pair of transmitter and receiver is assumed to experience uncorrelated stationary Rayleigh flat-fading. Hence, the square of the fading amplitudes $\left|h_{l}(t)\right|^{2}$ during the $t^{\text {th }}$ time slot (TS) obeys an exponential distribution having a unity mean, whose tail distribution

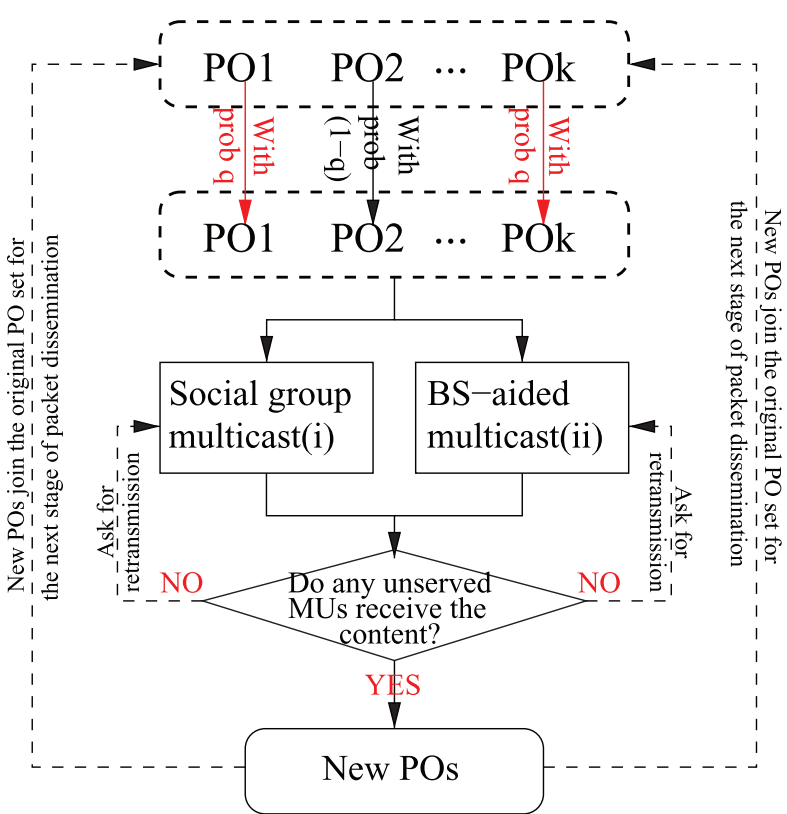

(i): Some POs are willing to further forward the content of common interest. (ii): None POs are willing to further forward the content of common interest.

Fig. 2. Actions of POs during the spontaneous content dissemination.

function $(\operatorname{tdf})$ is $\operatorname{Pr}\left[\left|h_{l}(t)\right|^{2}>x\right]=\mathrm{e}^{-x}$. Given an arbitrary 312 distance $y_{l}$ in meters, the path loss (PL) $\Omega_{l}$ is expressed as [27]: 313

$$
\Omega_{l}\left(y_{l}\right)= \begin{cases}1, & y_{l}<d_{0}, \\ \left(\frac{4 \pi f_{c}}{c}\right)^{\kappa} y_{l}^{\kappa}, & y_{l} \geq d_{0},\end{cases}
$$

where $c$ is the speed of light and $f_{c}$ is the carrier frequency, 314 whereas $\kappa$ is the PL exponent and $d_{0}$ is the distance from the 315 transmitter to the 'near-field' edge.

The random distance $Y_{l}$ is determined by the mobility pattern 317 of the MUs in the target social group. The following mobility 318 model is invoked for our performance analysis:

Definition 1 (Uniform mobility model): The position of the 320 $i^{\text {th }}$ MU during the $t^{\text {th }}$ time interval is denoted by $\mathbf{P}_{i}(t)$, which 321 obeys a stationary and ergodic process having a uniform dis- 322 tribution in the area considered. Moreover, the positions of 323 different MUs are independently and identically distributed 324 (i.i.d.).

This mobility model has been widely adopted for the per- 326 formance analysis of MANETs [28], [29]. Let the probability 327 density function (pdf) of the random distance $Y_{l}$ between any 328 two MUs be denoted by $f_{Y_{l}}\left(y_{l}\right)$. Our forthcoming performance 329 analysis is applicable not only to the uniform mobility model, 330 but to any arbitrary mobility model.

Note that, the index $l$ in the formulas is a generic subscript, 332 which represents ' $b$ ' when the BS is the transmitter, while it 333 represents ' $s$ ' when a MU is the transmitter. In the rest of the 334 paper, ' $l$ ', ' $b$ ' and ' $s$ ' hold the same meaning.

\section{Medium-Access-Control (MAC) Layer}

During a TS, a packet of the content is assumed to be suc- 337 cessfully received by a MU, provided that the instantaneous 338 
received signal-to-noise-ratio (SNR) is higher than a predefined threshold $\gamma$ [30]. In order to avoid collisions amongst multiple transmitters, orthogonal-frequency-divisionmultiple-access (OFDMA) or code-division-multiple-access (CDMA) may be invoked for allocating each transmitter an orthogonal channel. We denote the successful packet reception probability (SPRP) of a link as $\mu_{l}\left(y_{l}\right)$. By jointly considering the PHY layer model, the SPRP is derived as

$$
\begin{aligned}
\mu_{l}\left(y_{l}\right) & =\operatorname{Pr}\left(\frac{\operatorname{Pr}_{l}^{t x}\left|h_{l}(t)\right|^{2}}{\Omega_{l}\left(y_{l}\right) N_{0} W_{l}}>\gamma\right) \\
& = \begin{cases}\mathrm{e}^{-\frac{\gamma N_{0} W_{l}}{\operatorname{Pr}_{l}^{t x}}}, & y_{l}<d_{0}, \\
\mathrm{e}^{-\frac{\gamma N_{0} W_{l}}{\operatorname{Pr}_{l}^{t x}}\left(\frac{4 \pi f_{c}}{c}\right)^{\kappa} y_{l}^{\kappa}}, & y_{l} \geq d_{0},\end{cases}
\end{aligned}
$$

where $P_{l}^{t x}$ is the corresponding transmit power and $N_{0} W_{l}$ is the noise power in a communication bandwidth $W_{l}$. Given the pdf $f_{Y_{l}}\left(y_{l}\right)$ of the random distance $Y_{l}$, the average SPRP $\bar{\mu}_{l}$ of a link is derived as

$$
\begin{aligned}
\bar{\mu}_{l}= & \int_{0}^{d_{0}} \mathrm{e}^{-\frac{\gamma N_{0} W_{l}}{P_{l}^{t x}}} f_{Y_{l}}\left(y_{l}\right) d y_{l} \\
& +\int_{y_{l} \geq d_{0}} \mathrm{e}^{-\frac{\gamma N_{0} W_{l}}{P_{l}^{t x}}\left(\frac{4 \pi f_{c}}{c}\right)^{\kappa} y^{\kappa}} f_{Y_{l}}\left(y_{l}\right) d y_{l}
\end{aligned}
$$

Substituting the corresponding parameters and the pdf of the random distance into (6), we can obtain the average SPRP $\bar{\mu}_{s}$ between a pair of MUs and $\bar{\mu}_{b}$ between the BS and a MU. Moreover, the following lemma is proposed for our further analysis:

Lemma 1: Given the average SPRP $\bar{\mu}_{l}$ of a link during a TS, the average SPRP during a sufficiently short time interval $\Delta t$ ( $\Delta t \ll 1 \mathrm{TS}$ ) is approximately $\bar{\mu}_{l} \Delta t$.

Proof: The proof can be found in Appendix A.

Note that the SPRP also represents the normalized throughput, whose unit is packet/TS [30]. In more details, $\bar{\mu}_{l}$ indicates that $\bar{\mu}_{l}$ packets in average can be successfully received during a TS. Therefore, during $\Delta t(\leq 1) \mathrm{TS}$, only $\bar{\mu}_{l} \Delta t$ packets in average can be successfully received.

\section{Delay AnAlysis of the PACKET Dissemination}

In this section, various delay metrics of the packet dissemination process are derived with respect to a specific group size $N$. These metrics may replace the performance function $\mathcal{P}\left(\left|\mathcal{G}_{i}\right|=\right.$ $N)$ in (3) in order to characterize the average performance as a function of the content popularity.

\section{A. Pure Birth Markov Chain (PBMC)}

Let us assume that there are $N$ MUs in a considered social group. During the process of packet dissemination across the target social group, the number of POs steadily increases until all the $N$ social group members successfully receive the packet of common interest. Hence, the packet dissemination process can be modelled by a discrete-time PBMC having $(N+1)$ states, as shown in Fig. 3. In this PBMC, the states represent

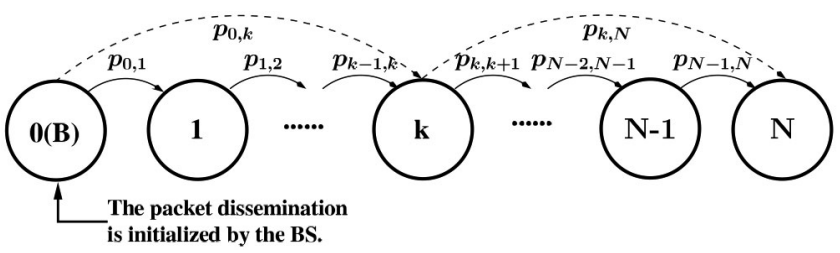

Fig. 3. A pure-birth Markov chain having an absorption state.

the corresponding numbers of POs having received the packet. 379 State transition only occurs from a lower-indexed state to a 380 higher-indexed one. Specifically, the state transition emerges 381 from state 0 , which represents the initial stage of the BS-aided 382 multicast, and terminates in state $N$, which indicates that all 383 the $N$ MUs in the target social group have received the desired 384 packet.

385

Let us first consider the general transition probability from 386 state $k$ to state $(k+m)$, where we have $1 \leq k \leq(N-1)$ and 387 $0 \leq m \leq(N-k)$. In the light of the selfish user-behaviour 388 considered, we assume that only $n_{k}, 1 \leq n_{k} \leq k$, POs are 389 willing to further disseminate the packet at the current stage. 390 Therefore, any unserved MU out of the $(N-k)$ unserved ones 391 is connected to the $n_{k}$ POs by $n_{k}$ wireless links, and any of 392 these links has the probability of $\bar{\mu}_{s} \Delta t$ to successfully deliver 393 the packet during the time interval $\Delta t$ according to Lemma 1. 394 As a result, given that $n_{k}$ POs independently deliver their pack- 395 ets to the same target, the SPRP of an unserved MU is expressed 396 as $\left[1-\left(1-\bar{\mu}_{s} \Delta t\right)^{n_{k}}\right]$. Furthermore, the state transition prob- 397

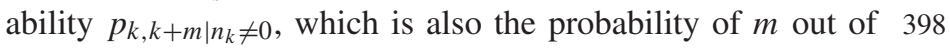
the $(N-k)$ unserved MUs successfully receiving the packet 399 during the current time interval $\Delta t$, can be expressed as

$$
\begin{aligned}
p_{k, k+m \mid n_{k} \neq 0}= & \left(\begin{array}{c}
N-k \\
m
\end{array}\right)\left[1-\left(1-\bar{\mu}_{s} \Delta t\right)^{n_{k}}\right]^{m} \\
& .\left(1-\bar{\mu}_{s} \Delta t\right)^{n_{k}(N-k-m)} \\
= & \left(\begin{array}{c}
N-k \\
m
\end{array}\right)\left[1-\sum_{i=0}^{n_{k}}\left(\begin{array}{c}
n_{k} \\
i
\end{array}\right)\left(-\bar{\mu}_{s} \Delta t\right)^{i}\right]^{m} \\
& .\left(1-\bar{\mu}_{s} \Delta t\right)^{n_{k}(N-k-m)} \\
= & \left(\begin{array}{c}
N-k \\
m
\end{array}\right)\left[\sum_{i=1}^{n_{k}}\left(\begin{array}{c}
n_{k} \\
i
\end{array}\right)(-1)^{i+1}\left(\bar{\mu}_{s} \Delta t\right)^{i}\right]^{m} \\
& .\left(1-\bar{\mu}_{s} \Delta t\right)^{n_{k}(N-k-m) .}
\end{aligned}
$$

According to (7), the state transition probability $p_{k, k+m \mid n_{k} \neq 0} 401$ has the same growth rate as $\bar{\mu}_{s}^{m} \Delta t^{m}$. Hence, the adjacent-state 402 transition probability $p_{k, k+1 \mid n_{k} \neq 0}$ of traversing from state $k$ to 403 state $(k+1)$ has the same growth rate as $\bar{\mu}_{s} \Delta t$. Substituting 404 $m=1$ into (7), $p_{k, k+1 \mid n_{k} \neq 0}$ can be expressed as

$$
\begin{aligned}
p_{k, k+1 \mid n_{k} \neq 0}=(N-k) n_{k} \bar{\mu}_{s} \Delta t \\
+(N-k)\left[\sum_{i=2}^{n_{k}(N-k-1)}\left(\begin{array}{c}
n_{k}(N-k-1) \\
i
\end{array}\right)\left(-\bar{\mu}_{s} \Delta t\right)^{i}\right. \\
\left.+\sum_{j=2}^{n_{k}(N-k)}\left(\begin{array}{c}
n_{k}(N-k) \\
j
\end{array}\right)\left(-\bar{\mu}_{s} \Delta t\right)^{j}\right] .
\end{aligned}
$$


TABLE II

PARAMETERS OF THE PHY LAYER

\begin{tabular}{|l|l|l|}
\hline & BS to MUs & MUs to MUs \\
\hline Transmit Power & $P_{b}^{t x}=31 \mathrm{dBm}$ & $P_{s}^{t x}=0 \sim 10 \mathrm{dBm}$ \\
\hline Carrier Freq & $f_{c, b}=1.8 \mathrm{GHz}$ & $f_{c, s}=2.4 \mathrm{GHz}$ \\
\hline Bandwidth & $W_{b}=10 \mathrm{MHz}$ & $W_{s}=10 \mathrm{MHz}$ \\
\hline Noise PSD & \multicolumn{2}{|c|}{$N_{0}=-174 \mathrm{dBm} / \mathrm{Hz}\left(20^{\circ} C\right)$} \\
\hline SNR Threshold & \multicolumn{2}{|c|}{$\gamma=10 \mathrm{~dB}$} \\
\hline PL Parameters & Exponent: $\kappa=3 ;$ Ref Distance: $d_{0}=1 \mathrm{~m}$ \\
\hline
\end{tabular}
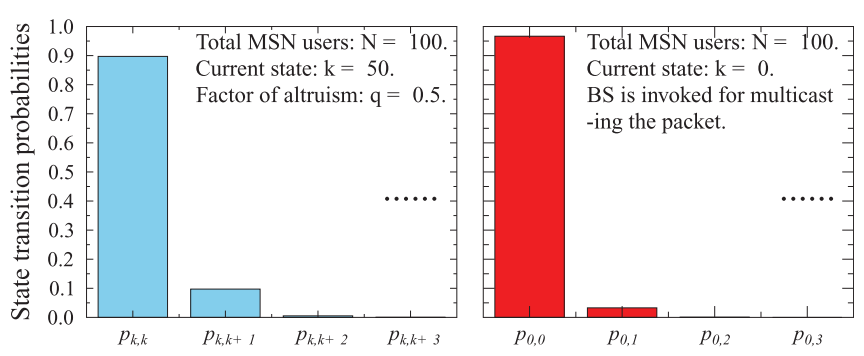

Fig. 4. State transition probabilities when $\Delta t=0.001 \mathrm{TS}$.

The terms in the square brackets of (8) have the same growth rate as $\bar{\mu}_{s}^{2} \Delta t^{2}$. Compared to the first term $(N-k) n_{k} \bar{\mu}_{s} \Delta t$ in (8), the terms in the square brackets are negligibly low, when $\bar{\mu}_{s} \Delta t$ is close to zero. Hence, in this case, we can approximate $p_{k, k+1 \mid n_{k} \neq 0}$ as $p_{k, k+1 \mid n_{k} \neq 0} \approx(N-k) n_{k} \bar{\mu}_{s} \Delta t$. Similarly, when $\bar{\mu}_{s} \Delta t$ is close to zero, $p_{k, k+m \mid n_{k} \neq 0}$ associated with $m \geq$ 2 in (7) can be approximated as $p_{k, k+m \mid n_{k} \neq 0} \approx 0$. Moreover, substituting $m=0$ into (7), we obtain the probability of the PBMC sojourning in the current state $k$ after the time interval $\Delta t$, which is $p_{k, k \mid n_{k} \neq 0}=\left(1-\bar{\mu}_{s} \Delta t\right)^{n_{k}(N-k)}$. Again, when $\bar{\mu}_{s} \Delta t$ is very close to zero, $p_{k, k \mid n_{k} \neq 0}$ can be approximated as $p_{k, k \mid n_{k} \neq 0} \approx 1-n_{k}(N-k) \bar{\mu}_{s} \Delta t$.

Another scenario is that no POs are willing to forward the packet, corresponding to the case $n_{k}=0$. Then the $(N-k)$ unserved MUs have to receive the packet directly from the BS. Similarly, we can also demonstrate that $p_{k, k+1 \mid n_{k}=0} \approx(N-k) \bar{\mu}_{b} \Delta t$ and $p_{k, k \mid n_{k}=0} \approx 1-(N-$ $k) \bar{\mu}_{b} \Delta t$, while $p_{k, k+m \mid n_{k}=0} \approx 0$ for $m \geq 2$, provided that $\bar{\mu}_{b} \Delta t$ is sufficiently small. Furthermore, it can be shown that $p_{0,1} \approx$ $N \bar{\mu}_{b} \Delta t, p_{0,0} \approx 1-N \bar{\mu}_{b} \Delta t$ and $p_{0, m} \approx 0$ for $m \geq 2$, provided that $\bar{\mu}_{b} \Delta t$ is sufficiently small.

According to the PHY layer parameters in TABLE II, we plot the state transition probabilities for state $k=50$ and for state $k=0$, respectively, in Fig. 4 . We observe from Fig. 4 that the state transition probabilities of $p_{k, k+m}$ and $p_{0, m}$ for $m \geq 2$ are negligibly low, which demonstrates the high accuracy of the above approximations involved.

Therefore, assuming a sufficiently short time interval $\Delta t$, only adjacent-state transitions occur during the process modelled by the discrete-time PBMC, as shown in Fig. 3.

\section{B. Delay of State Transition}

In order to study the delay statistics of disseminating a specific packet, we need to know the specific delay that the PBMC spends in a particular state, which is termed as the state transition delay. As a result, the following lemma may 440 be formulated:

Lemma 2: Given the state transition probability $\tilde{\mu}_{k} \Delta t$ from 442 the current state $k$ to state $(k+1)$, the transition delay from 443 state $k$ to state $(k+1)$ obeys the exponential distribution with a 444 mean of $1 / \tilde{\mu}_{k}$ TS, provided that $\Delta t$ is sufficiently small. Here, 445 $\tilde{\mu}_{k}$ is termed as the transition rate. 446

Proof: The proof can be found in Appendix B. 447

Based on Lemma 2, the discrete-time PBMC seen in Fig. 3448 can be further simplified to a continuous-time PBMC, which 449 only has adjacent-state transitions. The transition rate of this 450 continuous-time PBMC can be shown to be $p_{k, k+1} / \Delta t$, where 451 $p_{k, k+1}$ is the adjacent-state transition probability derived in 452 Section III-A.

Let us first consider the delay $T_{k}$ of the transition from state 454 $k$ to $(k+1)$, when $k \geq 1$. Since each PO has a probability $q$ of 455 forwarding the packet, in the current state $k$, the number $n_{k}(0 \leq 456$ $\left.n_{k} \leq k\right)$ of POs willing to forward the packet obeys a Binomial 457 distribution having a pair of parameters $k$ and $q$, whose pmf is 458 given by [31]

$$
p\left(n_{k}\right)=\left(\begin{array}{c}
k \\
n_{k}
\end{array}\right) q^{n_{k}}(1-q)^{k-n_{k}}, \quad n_{k}=0,1, \ldots, k
$$

For the case of $n_{k} \neq 0$, we have $p_{k, k+1 \mid n_{k} \neq 0} \approx n_{k}(N-460$ $k) \bar{\mu}_{s} \Delta t$. According to Lemma 2 , the delay $T_{k}$ of the transition 461 from state $k$ to state $(k+1)$ obeys an exponential distribu- 462 tion having a rate of $n_{k}(N-k) \bar{\mu}_{s}=n_{k} \mu_{s, k}$, where $\mu_{s, k}=463$ $(N-k) \bar{\mu}_{s}$. Hence, when $n_{k} \neq 0$, the conditional pdf, the mean 464 and the second moment of $T_{k}$ may be formulated as

$$
\begin{aligned}
f_{T_{k} \mid n_{k}}\left(t_{k}\right) & =n_{k} \mu_{s, k} \cdot \mathrm{e}^{-n_{k} \mu_{s, k} t_{k}}, t_{k} \geq 0 \\
\mathcal{E}\left[T_{k} \mid n_{k}\right] & =\int_{0}^{\infty} t_{k} f_{T_{k} \mid n_{k}}\left(t_{k}\right) d t_{k}=\frac{1}{n_{k} \mu_{s, k}}, \\
\mathcal{E}\left[T_{k}^{2} \mid n_{k}\right] & =\int_{0}^{\infty} t_{k}^{2} f_{T_{k} \mid n_{k}}\left(t_{k}\right) d t_{k}=\frac{2}{\left(n_{k} \mu_{s, k}\right)^{2}} .
\end{aligned}
$$

For the case of $n_{k}=0$, we have $p_{k, k+1 \mid n_{k}=0} \approx(N-466$ $k) \bar{\mu}_{b} \Delta t$, as the MUs in the target social group have to receive 467 the packet from the BS. According to Lemma 2, the delay $T_{k} 468$ of the transition from state $k$ to $(k+1)$ obeys an exponential 469 distribution having a rate of $\mu_{b, k}=(N-k) \bar{\mu}_{b}$. Hence, given 470 $n_{k}=0$, the conditional pdf, the mean and the second moment 471 of $T_{k}$ are derived as

$$
\begin{gathered}
f_{T_{k} \mid n_{k}=0}\left(t_{k}\right)=\mu_{b, k} \cdot \mathrm{e}^{-\mu_{b, k} t_{k}}, t_{k} \geq 0 \\
\mathcal{E}\left[T_{k} \mid n_{k}=0\right]=\int_{0}^{\infty} t_{k} f_{T_{k} \mid n_{k}=0}\left(t_{k}\right) d t_{k}=\frac{1}{\mu_{b, k}}, \\
\mathcal{E}\left[T_{k}^{2} \mid n_{k}=0\right]=\int_{0}^{\infty} t_{k}^{2} f_{T_{k} \mid n_{k}=0}\left(t_{k}\right) d t_{k}=\frac{2}{\mu_{b, k}^{2}} .
\end{gathered}
$$

According to the classic Bayesian principle [31], the pdf of $T_{k} 473$ may be expressed as 


$$
\begin{aligned}
f_{T_{k}}\left(t_{k}\right)= & \sum_{n_{k}=1}^{k} f_{T_{k} \mid n_{k}}\left(t_{k}\right) \cdot p\left(n_{k}\right)+f_{T_{k} \mid n_{k}=0}\left(t_{k}\right) \cdot p\left(n_{k}=0\right) \\
= & \sum_{n_{k}=1}^{k}\left(\begin{array}{c}
k \\
n_{k}
\end{array}\right) q^{n_{k}}(1-q)^{k-n_{k}} \cdot n_{k} \mu_{s, k} \mathrm{e}^{-n_{k} \mu_{s, k} t_{k}} \\
& \quad+(1-q)^{k} \mu_{b, k} \mathrm{e}^{-\mu_{b, k} t_{k}} .
\end{aligned}
$$

Moreover, the mean of $T_{k}$ is formulated as

$$
\begin{aligned}
\mathcal{E}\left[T_{k}\right] & =\mathcal{E}\left[T_{k} \mid n_{k}=0\right] p\left(n_{k}=0\right)+\sum_{n_{k}=1}^{k} \mathcal{E}\left[T_{k} \mid n_{k}\right] p\left(n_{k}\right) \\
& =\underbrace{\frac{(1-q)^{k}}{\mu_{b, k}}}_{\mathcal{E}\left[T_{k, b}\right]}+\underbrace{\sum_{n_{k}=1}^{k}\left(\begin{array}{c}
k \\
n_{k}
\end{array}\right) \frac{q^{n_{k}}(1-q)^{k-n_{k}}}{n_{k} \mu_{s, k}}}_{\varepsilon\left[T_{k, s}\right]}
\end{aligned}
$$

where $\mathcal{E}\left[T_{k, b}\right]$ represents the average duration of the BS-aided multicasting invoked during the transition from state $k$ to state $(k+1)$, where $\mathcal{E}\left[T_{k, s}\right]$ is the average duration of the social group multicasting during this state transition. Furthermore, the second moment of $T_{k}$ is formulated as

$$
\begin{aligned}
\mathcal{E}\left[T_{k}^{2}\right] & =\mathcal{E}\left[T_{k}^{2} \mid n_{k}=0\right] p\left(n_{k}=0\right)+\sum_{n_{k}=1}^{k} \mathcal{E}\left[T_{k}^{2} \mid n_{k}\right] p\left(n_{k}\right) \\
& =\frac{2(1-q)^{k}}{\mu_{b, k}^{2}}+\sum_{n_{k}=1}^{k}\left(\begin{array}{c}
k \\
n_{k}
\end{array}\right) \frac{2 q^{n_{k}}(1-q)^{k-n_{k}}}{\left(n_{k} \mu_{s, k}\right)^{2}}
\end{aligned}
$$

From (17) and (18), we can also derive the variance of $T_{k}$ by using the formula of $\operatorname{Var}\left[T_{k}\right]=\mathcal{E}\left[T_{k}^{2}\right]-\left\{\mathcal{E}\left[T_{k}\right]\right\}^{2}$. Furthermore, we may simply derive the pdf, the mean and the second moment of the transition delay $T_{0}$ from state 0 to state 1 by substituting $k=0$ in (13), (14), and (15), respectively.

\section{Dissemination Delay}

Since the delay of the transition from a state to its successor is independent of any other state transition's delay, and given that the dissemination delay across the target social group is defined as $T_{D}=\sum_{k=0}^{N-1} T_{k}$, the mean of $T_{D}$ can be expressed as

$$
\mathcal{E}\left[T_{D}\right]=\sum_{k=0}^{N-1} \frac{(1-q)^{k}}{\mu_{b, k}}+\sum_{k=1}^{N-1} \sum_{n_{k}=1}^{k}\left(\begin{array}{c}
k \\
n_{k}
\end{array}\right) \frac{q^{n_{k}}(1-q)^{k-n_{k}}}{n_{k} \mu_{s, k}},
$$

while the variance of $T_{D}$ can be formulated as $\operatorname{Var}\left[T_{D}\right]=$ $\sum_{k=0}^{N-1} \operatorname{Var}\left[T_{k}\right]$.

There is no exact closed-form tdf for the dissemination delay $T_{D}$ in this general case. However, given its mean and variance, we may approximate it as a random variable obeying the Gamma distribution, which is usually more accurate than its Gaussian counterpart, when non-negative random variables are concerned [32]. According to the theory of the Gamma distribution [33], it is uniquely and unambiguously described by its shape parameter $m=\left\{E\left[T_{D}\right]\right\}^{2} / \operatorname{Var}\left[T_{D}\right]$ and scale parameter $\Theta=\operatorname{Var}\left[T_{D}\right] / E\left[T_{D}\right]$. Then, given a delay threshold $D_{t h}$, we may derive the approximate probability of the dissemination delay $T_{D}$ exceeding $D_{t h}$ as

$$
\operatorname{Pr}\left(T_{D}>D_{t h}\right) \approx \frac{\Gamma\left(m, \frac{D_{t h}}{\Theta}\right)}{\Gamma(m)}=\frac{\Gamma\left(\frac{\left\{E\left[T_{D}\right]\right\}^{2}}{\operatorname{Var}\left[T_{D}\right]}, \frac{D_{t h} E\left[T_{D}\right]}{\operatorname{Var}\left[T_{D}\right]}\right)}{\Gamma\left(\frac{\left\{E\left[T_{D}\right]\right\}^{2}}{\operatorname{Var}\left[T_{D}\right]}\right)} .
$$

The accuracy of (20) will be verified by the Monte-Carlo 504 simulation in Section V.

\section{Individual User-Delay}

A specific MU $\mathcal{A}$ in the target social group may receive the 507 packet at any state spanning from 1 to $N$ during the process 508 of state transitions. When considering the transition from state 509 $(k-1)$ to $k(1 \leq k \leq N)$, any of the $(N-k+1)$ unserved 510 MUs may successfully receive the packet with a probability 511 of $1 /(N-k+1)$, and may not receive it with a probability of 512 $(N-k) /(N-k+1)$. Specifically, the probability of $\mathcal{A}$ receiv- 513 ing the packet in state $k$, which naturally implies that $\mathcal{A}$ has 514 not received the packets at any of the previous states, may be 515 expressed as

$$
p_{k}=\frac{1}{N-k+1} \cdot \prod_{i=1}^{k-1} \frac{N-i}{N-i+1}=\frac{1}{N}, \quad 1 \leq k \leq N .
$$

Hence, given that $\mathcal{A}$ receives the packet in state $k$, the 517 individual user-delay of $\mathcal{A}$ is expressed as $T_{\mathcal{A} \mid k}=\sum_{j=0}^{k-1} T_{k} 518$ and the conditional pdf of $T_{\mathcal{A} \mid k}$ is expressed as $f_{T_{\mathcal{A}} \mid k}\left(t_{\mathcal{A}}\right)=519$ $f_{T_{0}+\cdots+T_{k-1}}\left(t_{\mathcal{A}}\right)$. According to the Bayesian principle [31], the 520 pdf of the individual user-delay $T_{\mathcal{A}}$ can be expressed as:

$$
f_{T_{\mathcal{A}}}\left(t_{\mathcal{A}}\right)=\sum_{k=1}^{N} f_{T_{\mathcal{A}} \mid k}\left(t_{\mathcal{A}}\right) \cdot p_{k}=\sum_{k=1}^{N} \frac{f_{T_{0}+\cdots+T_{k-1}}\left(t_{\mathcal{A}}\right)}{N} .
$$

Furthermore, owing to the fact that $\left\{T_{0}, T_{1}, \ldots, T_{k-1}\right\}$ are inde- 522 pendent of each other, the average of $T_{\mathcal{A}}$ can be obtained 523 as

$$
\begin{aligned}
\mathcal{E}\left[T_{\mathcal{A}}\right] & =\int_{0}^{\infty} t_{\mathcal{A}} \sum_{k=1}^{N} \frac{f_{T_{0}+\cdots+T_{k-1}}\left(t_{\mathcal{A}}\right)}{N} d t_{\mathcal{A}}=\sum_{k=1}^{N} \frac{1}{N} \cdot \sum_{i=0}^{k-1} \mathcal{E}\left[T_{i}\right] \\
& =\sum_{k=1}^{N} \frac{N-k+1}{N} \mathcal{E}\left[T_{k-1}\right]
\end{aligned}
$$

where $\mathcal{E}\left[T_{k-1}\right]$ is given by (17). Furthermore, the second 525 moment of $T_{\mathcal{A}}$ is given by 
where $\boldsymbol{\xi}_{k}=\left(\mathcal{E}\left[T_{0}\right], \mathcal{E}\left[T_{1}\right], \ldots, \mathcal{E}\left[T_{k-1}\right]\right)^{T}, \quad \mathbf{H}_{k} \quad$ is a $k \times k$ matrix, whose elements are all ones, and $\mathbf{I}_{k}$ is a $k \times k$ identity matrix. Consequently, the variance of $T_{\mathcal{A}}$ can be expressed as $\operatorname{Var}\left(T_{\mathcal{A}}\right)=\mathcal{E}\left[T_{\mathcal{A}}^{2}\right]-\left\{\mathcal{E}\left[T_{\mathcal{A}}\right]\right\}^{2}$. Hence, by substituting $\mathcal{E}\left[T_{\mathcal{A}}\right]$ and $\operatorname{Var}\left[T_{\mathcal{A}}\right]$ into (20), we may obtain the approximate probability of $T_{\mathcal{A}}$ exceeding threshold $D_{t h}$.

\section{Delay Metrics for Special CASES}

\section{A. Case 1: Conventional BS-Aided Multicast $(q=0)$}

In this pessimistic case, all the MUs in the target social group are selfish during the packet dissemination process. Hence, the BS has to disseminate the packet to all the MUs in the target social group.

1) Dissemination Delay: When FA is $q=0$, according to Eqs.(13) (15) in Section III-B, the state transition delays $\left\{T_{k}, k=0,1, \ldots,(N-1)\right\}$ are the independent exponentially distributed variables associated with the rates of $\left\{\widetilde{\mu}_{k}=(N-\right.$ $\left.k) \bar{\mu}_{b}, k=0,1, \ldots,(N-1)\right\}$. Since the dissemination delay is defined as $T_{D}=\sum_{k=0}^{N-1} T_{k}, T_{D}$ obeys the hypoexponential distribution [34]. Furthermore, since the rates of $\left\{T_{k}, k=\right.$ $0,1, \ldots,(N-1)\}$ are different from each other, the pdf of $T_{D}$ can be expressed as

$$
f_{T_{D} \mid q=0}\left(t_{D}\right)=\sum_{k=0}^{N-1} \prod_{j=0, j \neq k}^{N-1} \frac{N-j}{k-j}(N-k) \bar{\mu}_{b} \mathrm{e}^{-(N-k) \bar{\mu}_{b} t_{D}} .
$$

In order to derive the probability of $T_{D}$ exceeding a given threshold $D_{t h}$, we integrate the above pdf $f_{T_{D} \mid q=0}\left(t_{D}\right)$ over the region $\left[D_{t h}, \infty\right)$, which is expressed as

$$
\begin{aligned}
\operatorname{Pr}\left(T_{D}>D_{t h} \mid q=0\right) & =\int_{D_{t h}}^{\infty} f_{T_{D} \mid q=0}\left(t_{D}\right) d t_{D} \\
& =\sum_{k=0}^{N-1} \prod_{j=0, j \neq k}^{N-1} \frac{N-j}{k-j} \mathrm{e}^{-(N-k) \bar{\mu}_{b} D_{t h}} .
\end{aligned}
$$

2) Individual User-Delay: When the FA is $q=0$, the individual user-delay is solely determined by the quality of the wireless link connecting the $\mathrm{MU} \mathcal{A}$ to the BS. As a result, according to Lemma 2 , the individual user-delay $T_{\mathcal{A}}$ obeys an exponential distribution having a mean of $1 / \bar{\mu}_{b}$. Furthermore, the probability of $T_{\mathcal{A}}$ exceeding a given threshold $D_{t h}$ is derived as $\operatorname{Pr}\left(T_{\mathcal{A}}>D_{t h} \mid q=0\right)=\exp \left(-\bar{\mu}_{b} D_{t h}\right)$.

\section{B. Case 2: Fully Altruistic Behaviours $(q=1)$}

In this optimistic scenario, all the MUs in the target social group are completely altruistic. Since there are always some POs willing to forward the packet during the dissemination process, the $\mathrm{BS}$ is not invoked for multicasting the packet any more, once some of the MUs have initially received it from the BS.

1) Dissemination Delay: When the FA is $q=1$, by substituting $n_{k}=k$ into Eqs.(10) (12) in Section III-B, we know that the state transition delays $\left\{T_{k}, k=1, \ldots,(N-567\right.$ 1) $\}$ are independent exponentially distributed variables associ- 568 ated with the rates of $\left\{\tilde{\mu}_{k}=k(N-k) \bar{\mu}_{s}, k=1,2, \ldots,(N-569\right.$ 1)\}. Furthermore, by substituting $k=0$ into Eqs.(13) (15) in 570 Section III-B, the initial state transition delay $T_{0}$ is also an expo- 571 nentially distributed variable associated with a rate of $\tilde{\mu}_{0}=572$ $N \bar{\mu}_{b}$. Note furthermore that $T_{0}$ is also independent of $\left\{T_{k}, k=573\right.$ $1, \ldots,(N-1)\}$. Since the dissemination delay is defined as 574 $T_{D}=\sum_{k=0}^{N-1} T_{k}, T_{D}$ obeys the hypoexponential distribution. 575

However, the rates of $\left\{\tilde{\mu}_{k}=k(N-k) \bar{\mu}_{s}, k=576\right.$ $1,2, \ldots,(N-1)\}$ associated with $\left\{T_{k}, k=1, \ldots,(N-1)\right\} 577$ exhibit a symmetric structure. For example, the rates of $T_{k} 578$ and $T_{N-k}$ share the same value of $k(N-k) \bar{\mu}_{s}$. Hence, the 579 closed-form equation for the tdf of $T_{D}$ may only be expressed 580 in the form of a continuous phase-type distribution [35]. As a 581 result, when $q=1$, the transition rate matrix of the PBMC is 582 expressed as

$$
\mathbf{P}=\left(\begin{array}{cccccc}
-\tilde{\mu}_{0} & \tilde{\mu}_{0} & 0 & \cdots & 0 & 0 \\
0 & -\tilde{\mu}_{1} & \tilde{\mu}_{1} & \ddots & 0 & 0 \\
\vdots & \ddots & -\tilde{\mu}_{k} & \tilde{\mu}_{k} & \ddots & \vdots \\
0 & 0 & \ddots & -\tilde{\mu}_{N-2} & \tilde{\mu}_{N-2} & 0 \\
0 & 0 & \ldots & 0 & -\tilde{\mu}_{N-1} & \tilde{\mu}_{N-1} \\
0 & 0 & \ldots & 0 & 0 & 0
\end{array}\right)=\left(\begin{array}{cc}
\mathbf{Q} & \mathbf{Q}_{0} \\
\mathbf{0} & 0
\end{array}\right),
$$

where $\mathbf{Q}$ is a $(N \times N)$-element matrix containing all the tran- 584 sition rates between transient states, $\mathbf{Q}_{0}$ is a $(N \times 1)$ column 585 vector containing all the transition rates from transient states to 586 the absorbing state $N$, whose last entry is $\widetilde{\mu}_{N-1}$ and finally, 587 the remaining entries are all zeros. As shown in Fig.3, the 588 packet dissemination process starts from the initial state 0. 589 Thus, the probability of $T_{D}$ exceeding a given threshold $D_{t h} 590$ is expressed as

$$
\operatorname{Pr}\left(T_{D} \geq D_{t h} \mid q=1\right)=\boldsymbol{\tau}_{1}^{T} \times \exp \left(D_{t h} \mathbf{Q}\right) \times \mathbf{1}_{N} .
$$

Note that in (28), the $(N \times 1)$ column vector $\boldsymbol{\tau}_{k+1}(0 \leq k \leq 592$ $N-1)$, whose $(k+1)$ th entry is one but all the others are 593 zeros, indicates that the PBMC starts at state $k$, while the 594 $(N \times 1)$ column vector $\mathbf{1}_{k+1}$, whose first $(k+1)$ entries are 595 ones and the remaining entries are zeros, indicates that the 596 PBMC process is absorbed at state $(k+1)$. The proof of $(28) 597$ can be found in [36].

2) Individual User-Delay: Given an event that the MU $\mathcal{A} 599$ successfully receives the packet at state $(k+1)(0 \leq k \leq N-600$ $1)$, the PBMC used for modelling the packet dissemination in 601 Fig. 3 is considered to be terminated at state $(k+1)$. According 602 to the physical meaning of both $\boldsymbol{\tau}_{k+1}$ and $\mathbf{1}_{k+1}$, similar to (28), 603 the probability of $T_{\mathcal{A}}$ exceeding the threshold $D_{t h}$, given that $\mathcal{A} 604$ receives the desired packet at state $(k+1)$ for $(0 \leq k \leq N-605$ 1 ), is expressed as

$$
\operatorname{Pr}\left(T_{\mathcal{A}} \geq D_{t h} \mid q=1, k+1\right)=\boldsymbol{\tau}_{1}^{T} \times \exp \left(D_{t h} \mathbf{Q}\right) \times \mathbf{1}_{k+1} .
$$

Since we have already derived the probability of $p_{k+1}=1 / N 607$ that $\mathcal{A}$ receives the packet at state $(k+1)$ in $(21)$, according to 608 the Bayesian principle [31], the probability of $T_{\mathcal{A}}$ exceeding the 609 


$$
\begin{aligned}
& \operatorname{Pr}\left(T_{\mathcal{A}} \geq D_{t h} \mid q=1\right)=\sum_{k=0}^{N-1} \operatorname{Pr}\left(T_{\mathcal{A}} \geq D_{t h} \mid q=1, k+1\right) \cdot p_{k+1} \\
& =\sum_{k=0}^{N-1} \frac{\boldsymbol{\tau}_{1}^{T} \times \mathbf{e x p}\left(D_{t h} \mathbf{Q}\right) \times \mathbf{1}_{k+1}}{N}=\frac{\boldsymbol{\tau}_{1}^{T} \times \exp \left(D_{t h} \mathbf{Q}\right)}{N} \times \sum_{k=0}^{N-1} \mathbf{1}_{k+1} \\
& =\frac{\boldsymbol{\tau}_{1}^{T} \times \exp \left(D_{t h} \mathbf{Q}\right) \times \eta}{N},
\end{aligned}
$$

where $\eta=(N, N-1, \ldots, 1)^{T}$ is a $(N \times 1)$ column vector.

\section{Case 3: Moderately Altruistic Behaviours $(q=0.5)$}

Unfortunately, we are unable to derive the exact tdf for the scenario, when the FA is set to $q=0.5$. However, we are still able to offer some interesting insights concerning the delay metrics of this specific case. Substituting $q=0.5$ into the second term of (17), the average duration of the social group multicast process during the transition from state $k$ to $(k+1)$ for $k \geq 1$ can be given by

$$
\mathcal{E}\left[T_{k, s} \mid q=0.5\right]=\frac{1}{2^{k} \cdot \mu_{s, k}} \sum_{n_{k}=1}^{k}\left(\begin{array}{c}
k \\
n_{k}
\end{array}\right) \frac{1}{n_{k}} .
$$

According to Eq.(68.1) of [33], we arrive at the following lower bound for $\mathcal{E}\left[T_{k, s} \mid q=0.5\right]$, which is expressed as:

$$
\begin{aligned}
\mathcal{E}\left[T_{k, s} \mid q=0.5\right] & >\frac{1}{2^{k} \cdot \mu_{s, k}}\left[\sum_{n_{k}=0}^{k}\left(\begin{array}{c}
k \\
n_{k}
\end{array}\right) \frac{1}{n_{k}+1}-1\right] \\
& =\frac{1}{2^{k} \cdot \mu_{s, k}} \frac{2^{k+1}-k+2}{k+1} .
\end{aligned}
$$

Similarly, substituting $q=1.0$ into the second term of (17), the corresponding formula of $\mathcal{E}\left[T_{k, s} \mid q=1.0\right]$ for this fully altruistic behaviour may be expressed as $\mathcal{E}\left[T_{k, s} \mid q=1.0\right]=$ $1 /\left(k \mu_{s, k}\right)$. As a result, the ratio $\mathcal{R}_{k, s}$ of these two expressions can be formulated as

$$
\mathcal{R}_{k, s}=\frac{\mathcal{E}\left[T_{k, s} \mid q=0.5\right]}{\mathcal{E}\left[T_{k, s} \mid q=1.0\right]}>\frac{\left(2^{k+1}-k+2\right) k}{2^{k}(k+1)} .
$$

In the ideal scenario, when $k$ tends to infinity, this ratio can be expressed as $\lim _{k \rightarrow \infty} R_{k, s}>2$. Since the lower bound derived in (32) is very tight ${ }^{8}$, we can summarise that by assuming moderately altruistic behaviours, the average duration of the social group multicasting during the transition from state $k$ to $(k+1)$ is twice that of the fully altruistic scenario, provided that $k$ is sufficiently high.

Let us now demonstrate the tightness of the lower bound (32) in terms of the average dissemination delay. Substituting (32) into (19), the lower bound of the average dissemination delay

\footnotetext{
${ }^{8}$ The tightness of this lower bound will be demonstrated in the following paragraph in terms of the average dissemination delay.
}

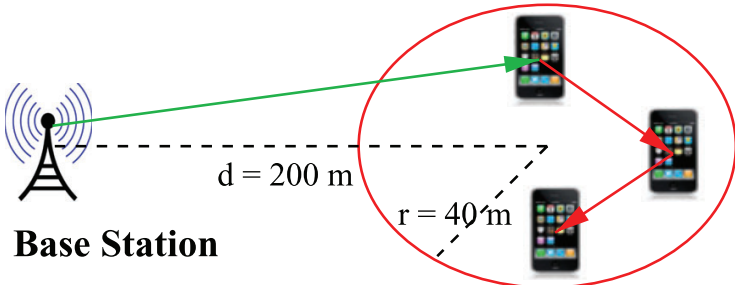

The Target MSN Group

Fig. 5. Geographic features for obtaining numerical results.

$\mathcal{E}\left[T_{D} \mid q=0.5\right]$ can be formulated as

$$
\begin{aligned}
\mathcal{E}\left[T_{D} \mid q=0.5\right] & =\sum_{k=0}^{N-1} \frac{1}{2^{k} \mu_{b, k}}+\sum_{k=1}^{N-1} \frac{1}{2^{k} \mu_{s, k}} \sum_{n_{k}=1}^{k}\left(\begin{array}{c}
k \\
n_{k}
\end{array}\right) \frac{1}{n_{k}} \\
& >\sum_{k=0}^{N-1} \frac{1}{2^{k} \mu_{b, k}}+\sum_{k=1}^{N-1} \frac{1}{2^{k} \cdot \mu_{s, k}} \frac{2^{k+1}-k+2}{k+1} .
\end{aligned}
$$

When we compute the exact result of $\mathcal{E}\left[T_{D} \mid q=0.5\right]$, which is 638 represented by the first line of (34), and its lower bound, which 639 is quantified by the second line of (34), then for a large social 640 group size $N$, such as $N=50 \sim 200$, using a set of other related 641 parameters in line with those of Fig. 6, the root-mean-square- 642 deviation (RMSD) of these two sets of results can be shown 643 to be 0.094 TS. Hence, we can claim that for a large social 644 group size, which represents our densely populated scenario, 645 the lower bound expressed in (34) can be regarded as an approx- 646 imate result of $\mathcal{E}\left[T_{D} \mid q=0.5\right]$. Furthermore, the tightness of the 647 lower bound derived in (33) can also be readily demonstrated. 648

Similarly, with the aid of (32), we can also obtain the lower 649 bound for the average individual user-delay $\mathcal{E}\left(T_{\mathcal{A}} \mid q=0.5\right)$.

\section{NUMERiCAl RESUlts}

The parameters of the PHY layer are presented in TABLE II. 652 The specific parameters used for transmissions from the BS 653 to the MUs are in line with FDD-LTE standard ${ }^{9}$, while the 654 transmission parameters between the MUs are in line with the 655 commonly used 802.11 protocol [18].

As shown in Fig. 5, we assume that all MUs in the target 657 social group roam in a circular area having a radius of $r=40658$ $\mathrm{m}$ by obeying the uniform mobility model. The BS is $d=200659$ $\mathrm{m}$ away from the centre of the circular area. In this scenario, 660 the pdf $f_{Y_{s}}\left(y_{s}\right)$ of the distance between a pair of MUs is given 661 by Eq. (23) of [38], and $f_{Y_{b}}\left(y_{b}\right)$ between the BS and a MU can 662 be found in our technical report [39]. Substituting $f_{Y_{s}}\left(y_{S}\right)$ and 663 $f_{Y_{b}}\left(y_{b}\right)$ into (6), alongside the parameters offered in TABLE II, 664 we may obtain the average SPRP $\bar{\mu}_{s}$ and $\bar{\mu}_{b}$, which further lead 665 us to the analytical (ana) results for the various metrics. If we 666 let $q=0$ in our model, the corresponding analytical results are 667 derived for conventional BS-aided multicast. $\quad 668$

In order to obtain a reliable statistical characterization of the 669 simulation performance (sim), we repeatedly run Monte-Carlo 670

\footnotetext{
${ }^{9}$ We assume a $1.8 \mathrm{GHz}$ carrier frequency in line with the LTE networks operated by the British company EE [37].
} 


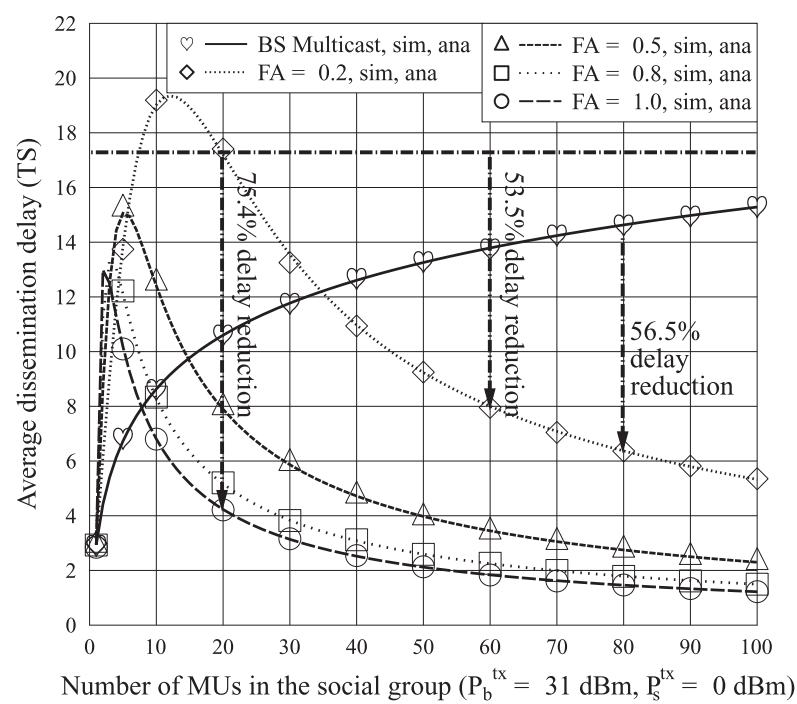

Fig. 6. Average dissemination delay affected by the number of MUs in the target social group, which is parameterized by the FA. The analytical results were evaluated from Eq. (19).

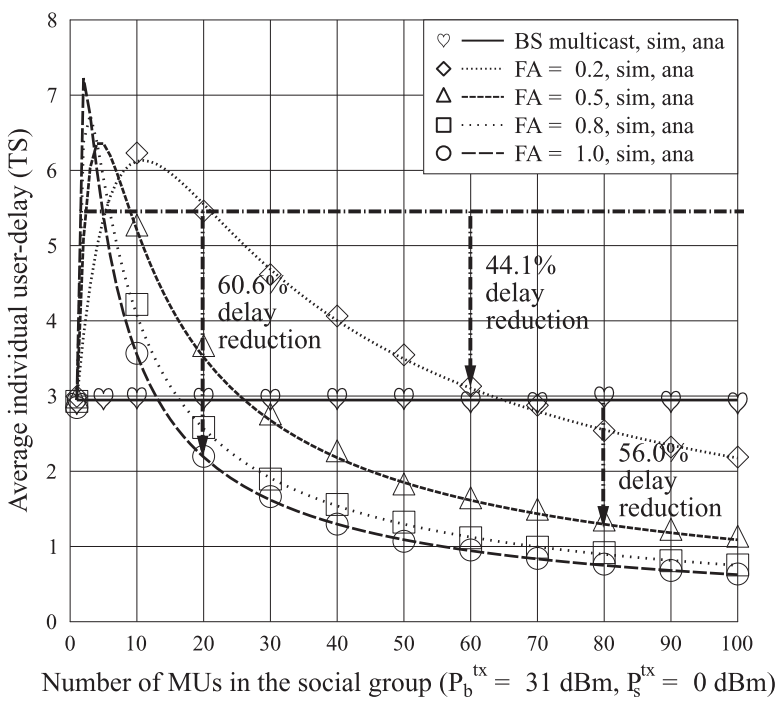

Fig. 7. Average individual user-delay as a function of the number of MUs in the target social group, which is parameterized by the FA. The analytical results were evaluated from (23).

simulations 10000 times and set the time-interval of our system to be $\Delta t=0.001 \mathrm{TS}$, where a TS can be considered as a packet duration. All the delay related metrics are evaluated by the number of TSs. In the numerical results of Figs. 6-8, we study the impact of the social group size $N$ on the delay metrics of the packet dissemination process without considering any specific content popularity.

\section{A. Delay Metrics for Uniform Mobility Model}

As shown in Fig. 6, when FA $\neq 0$, the average dissemination delay firstly increases, as the number of MUs is increased. When only a few MUs are in the target social group, a longer period is required for disseminating the packet to all of the group members due to the increasing content demand of the unserved MUs. However, by further increasing the number of

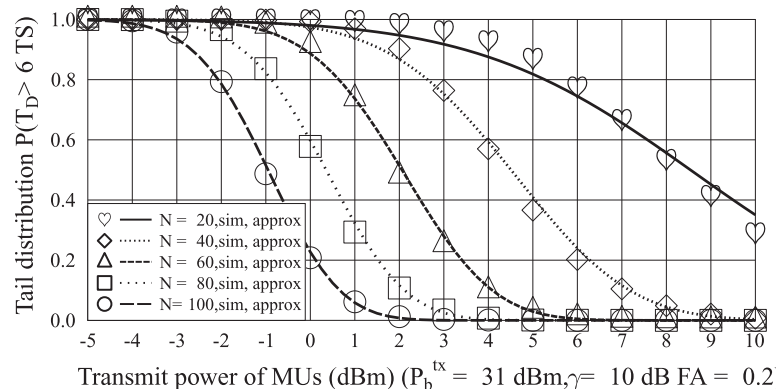

(a) Dissemination delay

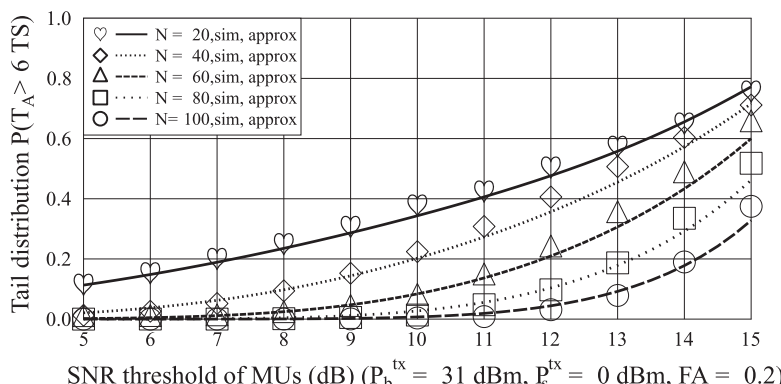

(b) Individual user-delay

Fig. 8. The tail distribution of the delay versus (a) the transmit power and (b) the SNR threshold for successful reception, which is parameterized by the number of MUs in the target social group. The analytical results were either directly or indirectly derived from Eq.(20).

MUs, the diversity gain incurred by the cooperation of the 685 multiple multicasters becomes sufficiently high to mitigate the 686 adverse effect of the increasing content demand. As a result, we 687 observe that the average dissemination delay decays after reach- 688 ing its peak, as the number of MUs is further increased. For 689 example, for $\mathrm{FA}=0.2$, the delay is reduced by $53.5 \%$, as the 690 number of MUs is increased from $N=20$ to 60. Furthermore, 691 a higher FA incurs a lower delay, since more POs are willing to 692 forward the packet after they successfully receive it. For exam- 693 ple, for $N=20$, the average dissemination delay is reduced 694 by $75.4 \%$, as the FA is increased from 0.2 to 1 . By contrast, 695 when $\mathrm{FA}=0$, the conventional BS-aided multicast technique 696 is invoked. However, as the number of the MUs increases, the 697 average dissemination delay also increases. We observe from 698 Fig. 6 that our approach is capable of reducing the average 699 dissemination delay of the conventional BS-aided multicast by 700 $56.5 \%$ for $N=80$, when a small FA value of 0.2 is assumed. 701

As shown in Fig. 7, when only a few MUs are in the tar- 702 get social group and the FA is non-zero, due to the users' 703 selfishness, fewer than two POs are willing to forward the 704 packet during the dissemination process. Therefore, we observe 705 from Fig. 7 that the average individual user-delay initially 706 increases, because it does not benefit from any diversity gain. 707 However, as we further increase the number of MUs, an increas- 708 ing number of POs become willing to forward the packet, 709 which substantially reduces the average individual user-delay, 710 as observed from Fig. 7. For example, for FA $=0.2$, the aver- 711 age individual user-delay is reduced by $44.1 \%$, as the number of 712 MUs is increased from $N=20$ to 60 . Nevertheless, when the 713 conventional BS-aided multicast is invoked, the average indi- 714 vidual user-delay, which only relies on the link connecting this 715 


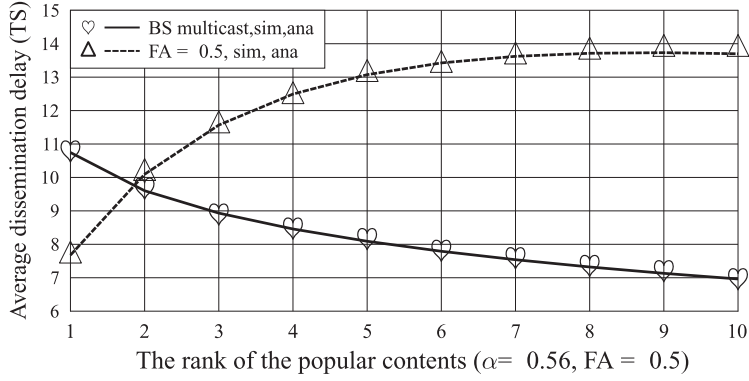

Fig. 9. Average dissemination delay as a function of the rank of the popular content. The transmit power of the BS is $P_{b}^{t x}=31 \mathrm{dBm}$ and the transmit power of a MU is $P_{t}^{t x}=0 \mathrm{dBm} . \mathcal{N}=100$ MUs independently request $\mathcal{M}=10$ ranked-popularity pieces of contents according to the request probabilities listed in TABLE I when $\alpha=0.56$. The analytical results were evaluated from Eq.(3).

specific MU to the BS, remains near-constant at 2.95 TS, as the number of MUs increases. Furthermore, the average individual user-delay is improved, when we increase the value of the FA. For example, given $N=20 \mathrm{MUs}$ in the target social group, the average individual user-delay is reduced by $60.6 \%$, as the FA is increased from 0.2 to 1.0. Additionally, given $N=80 \mathrm{MUs}$ in the target social group, the average individual user-delay drops from 2.95 TS to 1.3 TS, comparing the conventional BS-aided multicast to our approach associated with $\mathrm{FA}=0.5$.

Observe in Fig. 8(a) that the probability of the dissemination delay exceeding a threshold of $D_{t h}=6 \mathrm{TS}$ reduces upon increasing the transmit power of each MU. By contrast, as portrayed in Fig. 8(b), the probability of the individual userdelay exceeding the same threshold increases upon increasing the SNR threshold to be exceeded for ensuring successful packet reception. Our Gamma-distribution-based approximations match the simulation results.

Then, we study the average dissemination delay as a function of the specific popularity of the pieces of contents in Fig. 9. Observe from Fig. 9 that as a piece of contents becomes less popular, the average dissemination delay of our scheme increases, when we have a moderate degree of altruism associated with $\mathrm{FA}=0.5$. When a piece of content is less popular, fewer MUs may request this content, hence the resultant smaller social group fails to provide sufficient cooperative multicast opportunities for rapidly disseminating the packet across the social group. By contrast, since a less popular piece of contents results in a lower content demand, the average dissemination delay of the BS-aided multicast reduces, as the content becomes less popular. Furthermore, as shown in Fig. 9, our scheme associated with $\mathrm{FA}=0.5$ outperforms the conventional BS-aided multicast in terms of its delay of disseminating the most popular content. Nevertheless, the BS-adied multicast is more suitable for disseminating the less popular pieces of contents.

\section{B. Investigations Using Real Mobility Traces}

Let us now study the content dissemination performance in a densely-populated subway station scenario [40]. The mobility traces for this scenario can be downloaded from the CRAWDAD database ${ }^{10}$. The active area in this scenario is

\footnotetext{
${ }^{10}$ http://crawdad.cs.dartmouth.edu/kth/walkers/
}

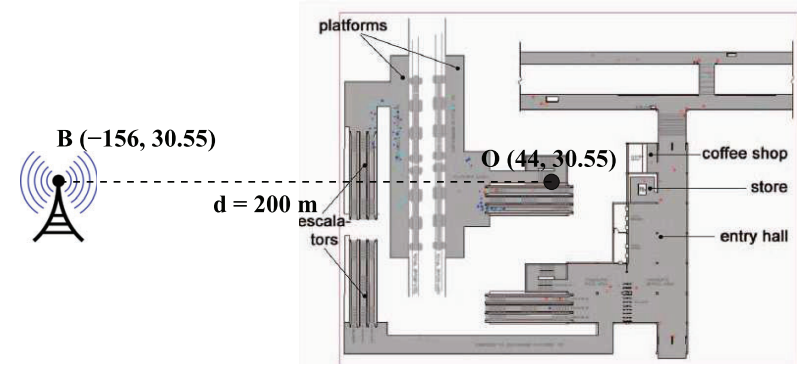

Fig. 10. A densely popluated subway station.

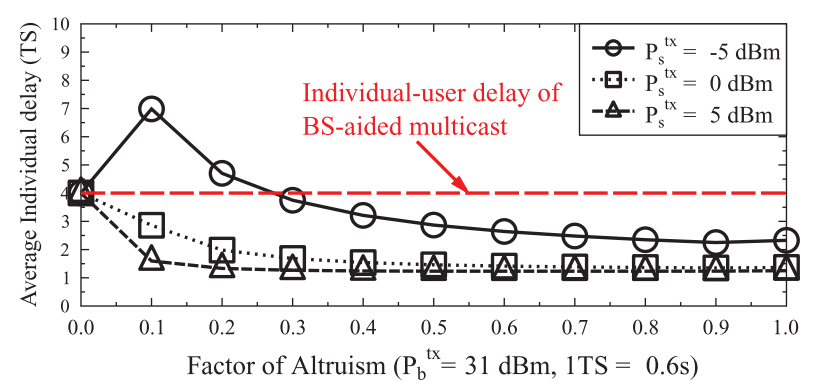

Fig. 11. Average individual user-delay in a subway station when all the MUs in the subway station form a grand social group for downloading a content of common interest.

$1921 \mathrm{~m}^{2}$. After analysing the mobility traces, the centre $O$ of 755 the active area is found to be at the coordinates of $(44,30.55) 756$ $\mathrm{m}$, as shown in Fig. 10. In our simulations, we placed the BS at 757 the point $(-156,30.55) \mathrm{m}$, which is $200 \mathrm{~m}$ away from the cen- 758 tre of the subway station. Since the MUs arrive/depart either 759 through the entrances or during the arrival/departure of trains, 760 the number of MUs is dynamic during the simulation time. As 761 a result, we cannot readily obtain the dissemination delay in 762 this scenario. However, we are still able to evaluate the indi- 763 vidual user-delay, when our content dissemination scheme and 764 conventional BS-aided multicast scheme are invoked. Again, 765 the physical layer parameters are summarised in TABLE II. 766 Since the positions of the MUs are captured every $0.6 \mathrm{~s}$ in this 767 mobility trace, in our simulations we set the basic time inter- 768 val of $\Delta t=0.6 \mathrm{~s}$ as a single TS, which can be considered as a 769 packet's duration. Then the delay was evaluated in terms of the 770 number of TSs.

We first assume that all the MUs in the subway station form 772 a large social group in order to download the train schedule of 773 common interest. Observe from Fig. 11 that for the cases of 774 $P_{S}^{t x}=0 \mathrm{dBm}$ and $P_{S}^{t x}=5 \mathrm{dBm}$, the average individual user- 775 delay is reduced, as we increase the FA from 0.0 to 1.0. For 776 $P_{s}^{t x}=-5 \mathrm{dBm}$, when $\mathrm{FA}$ is increased from 0.0 to 0.1 , we 777 observe an increasing average individual user-delay. This is 778 because the SPRP between the MUs is low and also, because 779 fewer POs are willing to forward the packet. As FA becomes 780 higher, more POs may join to assist the packet dissemina- 781 tion process, which significantly reduces the average individual 782 user-delay. Specifically, when FA $=0$, conventional BS-aided 783 multicast is invoked for disseminating the packets. For $P_{s}^{t x}=0 \quad 784$ or $5 \mathrm{dBm}$, if the MUs become only modestly altruistic, say we 785 have $\mathrm{FA}=0.1$, our content dissemination scheme outperforms 786 


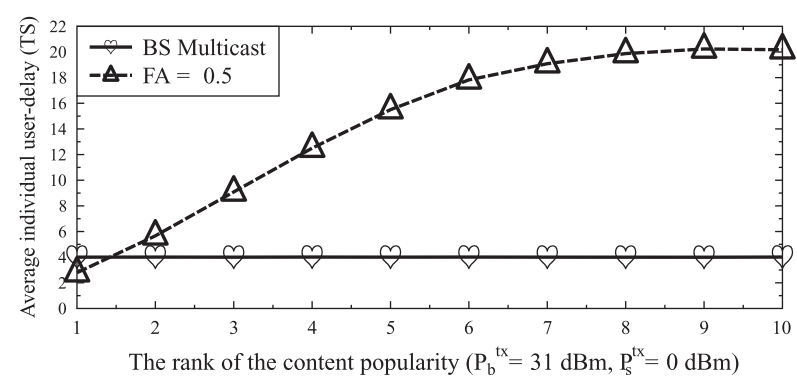

Fig. 12. Average individual user-delay in a subway station when the MUs in the subway station independently request $\mathcal{M}=10$ ranked-popularity pieces of contents according to the probabilities listed in TABLE I when $\alpha=1$.

A TS, during which the successful packet reception probability is $\mu_{i}$

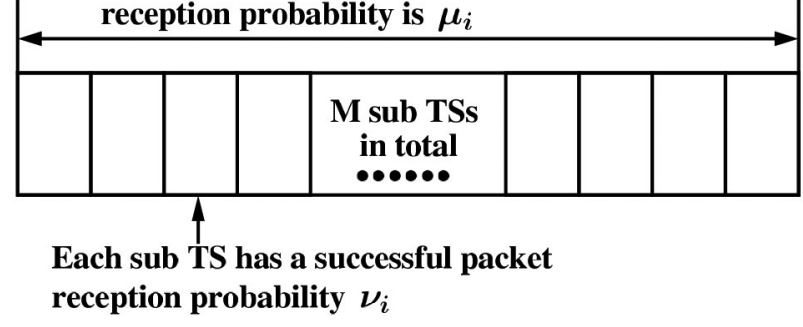

Fig. 13. The structure of a TS.

the conventional BS-aided multicast. For $P_{s}^{t x}=-5 \mathrm{dBm}$, our scheme starts to outperform the classic BS-aided multicast, provided that FA is higher than 0.4 .

We then study the impact of the specific content popularity on the average individual user-delay in a subway station. Observe from Fig. 12 that when disseminating the most popular content in the subway station, our dissemination scheme associated with $\mathrm{FA}=0.5$ outperforms the conventional BS-aided multicast. However, the BS-aided multicast is more suitable for disseminating less popular content in this scenario. The reason behind this trend is the same as that associated with Fig. 9.

\section{Vi. Conclusions}

In this paper, we proposed a social group multicast aided content dissemination scheme as a supplement to the conventional cellular system. The content popularity is modelled by a Zipf distribution and the concept of FA was introduced for the sake of quantifying the probability of a PO forwarding a packet of the content of common interest. In our scheme, the BSs are invoked for multicasting the packet at the initial stage, as well as when no POs are willing to share the packet with others. By modelling the packet dissemination process as a PBMC, closed-form expressions were derived for the statistical properties of the various delay metrics. We demonstrated that our approach outperforms the conventional BS-adied multicast in terms of both the dissemination delay and the individual-user delay, especially when the density of MUs in a target group is high. Furthermore, we found that our approach is more suitable for disseminating a more popular content. By contrast, the conventional BS-aided multicast performs better for disseminating a less popular content.
APPENDIX A

As shown in Fig. 13, a TS is divided into $M$ sub-TSs, each 819 of which has a duration of $\Delta t=1 / M$ TS. We assume that the 820 SPRP in a sub-TS is $\nu_{i}$. As a result, given the SPRP $\bar{\mu}_{i}$ in a 821 TS, we may derive the relation between $\bar{\mu}_{i}$ and $v_{i}$, which is 822 expressed as

$$
\bar{\mu}_{i}=\sum_{j=1}^{M}\left(1-v_{i}\right)^{j-1} v_{i}=1-\left(1-v_{i}\right)^{M} .
$$

Rewriting the above expression, we obtain

$$
v_{i}=1-\left(1-\bar{\mu}_{i}\right)^{1 / M}=1-\left(1-\bar{\mu}_{i}\right)^{\Delta t},
$$

where the second equality is derived according to $\Delta t=1 / M \quad 825$ TS. If we expand $\left(1-\bar{\mu}_{i}\right)^{\Delta t}$ according to the Taylor series, we 826 have

$$
\left(1-\bar{\mu}_{i}\right)^{\Delta t}=\sum_{n=0}^{\infty}\left(\begin{array}{c}
\Delta t \\
n
\end{array}\right)\left(-\bar{\mu}_{i}\right)^{n}=1-\bar{\mu}_{i} \Delta t+O\left(\bar{\mu}_{i}^{2}\right),
$$

where $O\left(\bar{\mu}_{i}^{2}\right)$ is the infinitesimal by small quantity on the same 828 order as $\bar{\mu}_{i}^{2}$. Substituting the above equation into (36), we have 829

$$
v_{i}=\bar{\mu}_{i} \Delta t+O\left(\bar{\mu}_{i}^{2}\right) \approx \bar{\mu}_{i} \Delta t .
$$

According to our experiments, if we vary $\bar{\mu}_{i}$ from 0 to $0.8,830$ the root-mean-square-deviation (RMSD) between the exact $v_{i} 831$ given by (36) and the approximated $v_{i}$ given by $(38)$ is $9.45 \times 832$ $10^{-4}$. As a result, it is reasonable to claim that $\nu_{i} \approx \bar{\mu}_{i} \Delta t$.

APPENDIX B

The Proof OF LEMMA 2

During a time interval $\Delta t$, the PBMC may transit from state 836 $k$ to $(k+1)$ with a probability of $\tilde{\mu}_{k} \Delta t$. Naturally, the success- 837 ful state transition first occurring during the $\left(M_{k}=m_{k}\right)$-th $\Delta t \quad 838$ interval obeys a geometric distribution. According to the PMF 839 of a geometric distribution having a parameter of $\tilde{\mu}_{k} \Delta t$, we 840 arrive at:

$$
\begin{gathered}
\operatorname{Pr}\left(M_{k} \Delta t \leq m_{k} \Delta t\right)=\sum_{m=1}^{m_{k}}\left(1-\tilde{\mu}_{k} \Delta t\right)^{m-1} \tilde{\mu}_{k} \Delta t \\
\operatorname{Pr}\left(M_{k} \Delta t \leq\left(m_{k}+1\right) \Delta t\right)=\sum_{m=1}^{m_{k}+1}\left(1-\tilde{\mu}_{k} \Delta t\right)^{m-1} \tilde{\mu}_{k} \Delta t .
\end{gathered}
$$

The continuous-valued delay of the adjacent-state transition is 842 denoted as $T_{k}=M_{k} \Delta t$, which is associated with a specific 843 value of $t_{k}=m_{k} \Delta t$. Hence, we may derive the pdf of $T_{k}$ as: 844

$$
\begin{aligned}
f_{T_{k}}\left(t_{k}\right) & =\lim _{\Delta t \rightarrow 0} \frac{\operatorname{Pr}\left(T_{k} \leq t_{k}+\Delta t\right)-\operatorname{Pr}\left(T_{k} \leq t_{k}\right)}{\Delta t} \\
& =\lim _{\Delta t \rightarrow 0} \frac{\operatorname{Pr}\left(M_{k} \Delta t \leq\left(m_{k}+1\right) \Delta t\right)-\operatorname{Pr}\left(M_{k} \Delta t \leq m_{k} \Delta t\right)}{\Delta t} \\
& =\lim _{\Delta t \rightarrow 0} \frac{\left(1-\tilde{\mu}_{k} \Delta t\right)^{m_{k}} \tilde{\mu}_{k} \Delta t}{\Delta t}=\lim _{\Delta t \rightarrow 0} \tilde{\mu}_{k} \mathrm{e}^{-m_{k} \tilde{\mu}_{k} \Delta t} \\
& =\tilde{\mu}_{k} \cdot \mathrm{e}^{-\tilde{\mu}_{k} t_{k}},
\end{aligned}
$$


845 where the last two lines are derived based on $\lim _{\Delta t \rightarrow 0} \tilde{\mu}_{k} \Delta t=$ $1-\mathrm{e}^{-\widetilde{\mu}_{k} \Delta t}$ and $m_{k}=t_{k} / \Delta t$, respectively.

\section{REFERENCES}

[1] N. Kayastha, D. Niyato, P. Wang, and E. Hossain, "Applications, architectures, and protocol design issues for mobile social networks: A survey," Proc. IEEE, vol. 99, no. 12, pp. 2130-2158, Dec. 2011.

[2] A. Vahdat and D. Becker, "Epidemic routing for partially-connected ad hoc networks," Master thesis, Dept. Comput. Sci., Duke Univ., Durham, NC 27708 USA, Tech. Rep., 2000.

[3] Y.-K. Ip, W.-C. Lau, and O.-C. Yue, "Performance modeling of epidemic routing with heterogeneous node types," in Proc. IEEE Int. Conf. Commun. (ICCŠO8), May 2008, pp. 219-224.

[4] A. Clementi, F. Pasquale, and R. Silvestri, "Opportunistic manets: Mobility can make up for low transmission power," IEEE/ACM Trans. Netw., vol. 21, no. 2, pp. 610-620, Feb. 2013.

[5] H. Sun and C. Wu, "Epidemic forwarding in mobile social networks," in Proc. IEEE Int. Conf. Commun. (ICC'12), Jun. 2012, pp. 1421-1425.

[6] J. Whitbeck, V. Conan, and M. D. de Amorim, "Performance of opportunistic epidemic routing on edge-markovian dynamic graphs," IEEE Trans. Commun., vol. 59, no. 5, pp. 1259-1263, May 2011.

[7] S. Ioannidis, A. Chaintreau, and L. Massoulie, "Optimal and scalable distribution of content updates over a mobile social network," in Proc. IEEE INFOCOM, 2009, pp. 1422-1430.

[8] C. Boldrini, M. Conti, and A. Passarella, "Contentplace: Social-aware data dissemination in opportunistic networks," in Proc. 11th Int. Symp. Model. Anal. Simul. Wireless Mobile Syst. (MSWiM'08), 2008, pp. 203210.

[9] D. Niyato, P. Wang, W. Saad, and A. Hj申andrungnes, "Controlled coalitional games for cooperative mobile social networks," IEEE Trans. Veh. Technol., vol. 60, no. 4, pp. 1812-1824, May 2011.

[10] K. Akkarajitsakul, E. Hossain, and D. Niyato, "Cooperative packet delivery in hybrid wireless mobile networks: A coalitional game approach," IEEE Trans. Mobile Comput., vol. 12, no. 5, pp. 1-15, May 2013.

[11] B. Han, P. Hui, V. Kumar, M. Marathe, J. Shao, and A. Srinivasan, "Mobile data offloading through opportunistic communications and social participation," IEEE Trans. Mobile Comput., vol. 11, no. 5, pp. 821-834, May 2012.

[12] Y. Li, M. Qian, D. Jin, P. Hui, Z. Wang, and S. Chen, "Multiple mobile data offloading through disruption tolerant networks," IEEE Trans. Mobile Comput., vol. 13, no. 7, pp. 1579-1596, Jul. 2014.

[13] R. Groenevelt, P. Nain, and G. Koole, "The message delay in mobile ad hoc networks," Perform. Eval., vol. 62, nos. 1-4, pp. 210-228, Oct. 2005.

[14] T. Karagiannis, J.-Y. Le Boudec, and M. Vojnovic, "Power law and exponential decay of intercontact times between mobile devices," IEEE Trans. Mobile Comput., vol. 9, no. 10, pp. 1377-1390, Oct. 2010.

[15] J. Hu, L.-L. Yang, and L. Hanzo, "Mobile social networking aided content dissemination in heterogeneous networks," China Commun., vol. 10, no. 6, p. 1, 2013.

[16] J. Wang, S. Park, D. Love, and M. Zoltowski, "Throughput delay tradeoff for wireless multicast using hybrid-ARQ protocols," IEEE Trans. Commun., vol. 58, no. 9, pp. 2741-2751, Sep. 2010

[17] J. Seo, T. Kwon, and V. Leung, "Social groupcasting algorithm for wireless cellular multicast services," IEEE Commun. Lett., vol. 17, no. 1, pp. 47-50, Jan. 2013.

[18] Information Technology-Telecommunications and Information Exchange Between Systems Local and Metropolitan Area NetworksSpecific Requirements Part 11: Wireless LAN Medium Access Control (MAC) and Physical Layer (PHY) Specifications, ISO/IEC/IEEE Standard 8802-11:2012(E), Nov. 2012, pp. 1-2798.

[19] Z. Gong and M. Haenggi, "Interference and outage in mobile random networks: Expectation, distribution, and correlation," IEEE Trans. Mobile Comput., vol. 13, no. 2, pp. 337-349, Feb. 2014.

[20] H. Kwon and B. G. Lee, "Cooperative power allocation for broadcast/ multicast services in cellular OFDM systems," IEEE Trans. Commun., vol. 57, no. 10, pp. 3092-3102, Oct. 2009.

[21] D. Feng, L. Lu, Y. Yuan-Wu, G. Li, G. Feng, and S. Li, "Device-to-device communications underlaying cellular networks," IEEE Trans. Commun., vol. 61, no. 8, pp. 3541-3551, Aug. 2013.

[22] R. E. Hattachi and J. Erfanian, "NGMN 5G White Paper version 1.0," Next Generation Mobile Networks (NGMN), NGMN Alliance, Frankfurt, Germany, 2015.
[23] M. Cha, H. Kwak, P. Rodriguez, Y.-Y. Ahn, and S. Moon, "I tube, you 916 tube, everybody tubes," in Proc. 7th ACM SIGCOMM Conf. Internet 917 Meas. (IMC'07), Oct. 2007, p. 1.

[24] M. Zink, K. Suh, Y. Gu, and J. Kurose, "Characteristics of YouTube network traffic at a campus network-Measurements, models, and implications," Comput. Netw., vol. 53, no. 4, pp. 501-514, Mar. 2009.

[25] K. Shanmugam, N. Golrezaei, A. G. Dimakis, A. F. Molisch, and 922 G. Caire, "FemtoCaching: Wireless content delivery through distributed 923 caching helpers," IEEE Trans. Inf. Theory, vol. 59, no. 12, pp. 8402-8413, 924 Dec. 2013.

[26] M. Taghizadeh, K. Micinski, S. Biswas, C. Ofria, and E. Torng, 926 "Distributed cooperative caching in social wireless networks," IEEE 927 Trans. Mobile Comput., vol. 12, no. 6, pp. 1037-1053, Jun. 2013.

[27] T. Rappaport, Wireless Communications: Principles and Practice, 2nd ed. 929 Upper Saddle River, NJ, USA: Prentice-Hall, 2001.

[28] H. Zhang, Z. Zhang, and H. Dai, "Gossip-based information spreading in mobile networks," IEEE Trans. Wireless Commun., vol. 12, no. 11, pp. 5918-5928, Nov. 2013.

[29] X. Wang, Q. Peng, and Y. Li, "Cooperation achieves optimal multi- 934 cast capacity-delay scaling in MANET," IEEE Trans. Commun., vol. 60, 935 no. 10, pp. 3023-3031, Oct. 2012.

[30] J. Hu, L.-L. Yang, and L. Hanzo, "Maximum average service rate and 937 optimal queue scheduling of delay-constrained hybrid cognitive radio in 938 Nakagami fading channels," IEEE Trans. Veh. Technol., vol. 62, no. 5, 939 pp. 2220-2229, Jun. 2013.

[31] P. V. Mieghem, Performance Analysis of Communications Networks and Systems. Cambridge, U.K.: Cambridge Univ. Press, 2005.

[32] M. Derakhshani and T. Le-Ngoc, "Aggregate interference and capacityoutage analysis in a cognitive radio network," IEEE Trans. Veh. Technol., vol. 61, no. 1, pp. 196-207, Jan. 2012.

[33] I. S. Gradshteyn and I. M. Ryzhik, Table of Integrals, Series, and 946 Products, 7th ed. New York, NY, USA: Elsevier/Academic, 2007.

[34] W. Gao, Q. Li, B. Zhao, and G. Cao, "Social-aware multicast in 948 disruption-tolerant networks," IEEE/ACM Trans. Netw., vol. 20, no. 5, 949 pp. 1553-1566, Oct. 2012.

[35] M. W. Fackrell, "Characterization of matrix-exponential distributions," 951 Ph.D. dissertation, Faculty of Eng., Comput. Math. Sci., School of Appl. 952 Math., Univ. Adelaide, Adelaide, South Australia, 5005, Australia, 2003. 953

[36] G. Latouche and V. Ramaswami, Introduction to Matrix Analytic Methods 954 in Stochastic Modeling. Philadelphia, PA, USA: SIAM, 1999.

[37] Office of Communications. "Notice of proposed variation of Everything 956 Everywhere's $1800 \mathrm{MHz}$ spectrum licences to allow use of LTE 957 and WiMAX technologies," Ofcom, Mar. 2012 [Online]. Available: 958 http://stakeholders.ofcom.org.uk/consultations/variation-1800mhz-lte- $\quad 959$ wimax/summary.

[38] C. Bettstetter, H. Hartenstein, and X. Pérez-Costa, "Stochastic properties of the random waypoint mobility model," Wireless Netw., vol. 10, no. 5 , pp. 555-567, 2004

[39] J. Hu, L.-L. Yang, and L. Hanzo, "Stochastic geometry in the cellular networks," Univ. Southampton, SO17 1BJ, U.K., Tech. Rep., 2015 [Online]. Available: http://eprints.soton.ac.uk/id/eprint/374932.

[40] O. Helgason, S. T. Kouyoumdjieva, and G. Karlsson, "Opportunistic communication and human mobility," IEEE Trans. Mobile Comput., vol. 13, no. 7, pp. 1597-1610, Jul. 2014.

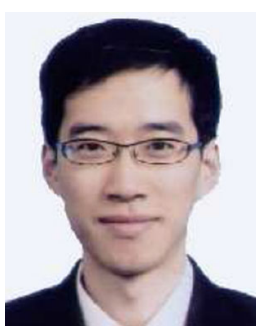

Jie Hu (S'11-M'16) received the B.Eng. degree 970 in communication engineering and the M.Eng. 971 degree in communication and information sys- 972 tem from the School of Communication and 973 Information Engineering, Beijing University of Posts 974 and Telecommunications, Beijing, China, in 2008975 and 2011, respectively, and the Ph.D. degree from 976 the Southampton Wireless Group, University of 977 Southampton, Southampton, U.K., in 2015.

Since March 2016, he has been working with 979 the School of Communication and Information 980 Engineering, University of Electronic Science and Technology of China 981 (UESTC), Chengdu, China, as a Lecturer. He has a broad range of interests 982 in wireless communication and networking, such as cognitive radio and cog- 983 nitive networks, queuing analysis, resource allocation and scheduling, ad hoc 984 wireless networks, and mobile social networks. 
986

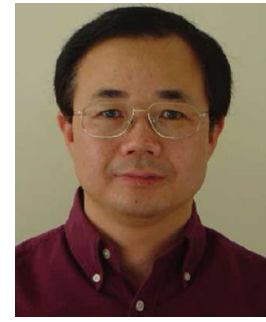

Lie-Liang Yang (M'98-SM'02-F'16) received the B.Eng. degree in communications engineering from Shanghai TieDao University, Shanghai, China, in 1988, and the M.Eng. and Ph.D. degrees in communications and electronics from Beijing (Northern) Jiaotong University, Beijing, China, in 1991 and 1997, respectively. From June 1997 to December 1997, he was a Visiting Scientist at the Institute of Radio Engineering and Electronics, Academy of Sciences of the Czech Republic, Prague, Czech Republic. Since December 1997, he has been with the University of Southampton, Southampton, U.K., where he is currently a Professor of Wireless Communications with the School of Electronics and Computer Science. His research interests include wireless communications, wireless networking, and signal processing for wireless communications. $\mathrm{He}$ has authored more than 300 research papers in journals and conference proceedings, authored or coauthored three books, and also published several book chapters.

Prof. Yang is a Fellow of The Institution of Engineering and Technology, U.K. He served as an Associate Editor for the IEEE TRANSACTIONS ON VEHICULAR TECHNOLOGY and the Journal of Communications and Networks, and is currently an Associate Editor of the IEEE ACCESS and the Security and Communication Networks Journal.

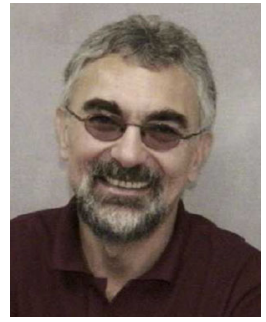

Lajos Hanzo (M'91-SM'92-F'03) received the 1009 degree in electronics, in 1976, the Doctorate degree, 1010 in 1983, and the D.Sc. degree. In 2009, he received 1011 the Honorary Doctorate "Doctor Honoris Causa" 1012 from the Technical University of Budapest, Budapest, 1013 Hungary. During his 35-year career in telecommuni- 1014 cations, he has held various research and academic 1015 posts in Hungary, Germany, and the U.K. Since 1016 1986, he has been with the School of Electronics 1017 and Computer Science, University of Southampton, 1018 Southampton, U.K., where he holds the Chair in 1019 telecommunications. He has successfully supervised 80 Ph.D. students, coau- 1020 thored 20 John Wiley/IEEE Press books on mobile radio communications 1021 totalling in excess of 10,000 pages, published over 1500 research entries at 1022 IEEE Xplore, acted both as TPC and General Chair of the IEEE conferences, 1023 presented keynote lectures and has been awarded a number of distinctions. 1024 Currently, he is directing a 60-strong academic research team, working on a 1025 range of research projects in the field of wireless multimedia communications 1026 sponsored by industry, the Engineering and Physical Sciences Research Council 1027 (EPSRC) U.K., the European IST Programme and the Mobile Virtual Centre of 1028 Excellence (VCE), U.K. He is an enthusiastic supporter of industrial and aca- 1029 demic liaison and he offers a range of industrial courses. He is also a Governor 1030 of the IEEE VTS. From 2008 to 2012, he was the Editor-in-Chief of the IEEE 1031 Press and a Chaired Professor also at Tsinghua University, Beijing, China. His 1032 research is funded by the European Research Council's Senior Research Fellow 1033 Grant. He is the Fellow of the REng, IET, and EURASIP. 


\title{
Delay Analysis of Social Group Multicast-Aided Content Dissemination in Cellular System
}

\author{
Jie Hu, Member, IEEE, Lie-Liang Yang, Fellow, IEEE, and Lajos Hanzo, Fellow, IEEE
}

\begin{abstract}
Based on the common interest of mobile users (MUs) in a social group, the dissemination of content across the social group is studied as a powerful supplement to conventional cellular communication with the goal of improving the delay performance of the content dissemination process. The content popularity is modeled by a Zipf distribution to characterize the MUs' different interests in different contents. The factor of altruism (FA) terminology is introduced for quantifying the willingness of content owners to share their content. We model the dissemination process of a specific packet by a pure-birth-based Markov chain and evaluate the statistical properties of both the network's dissemination delay as well as of the individual user-delay. Compared to the conventional base station (BS)-aided multicast, our scheme is capable of reducing the average dissemination delay by about $56.5 \%$. Moreover, in contrast to the BS-aided multicast, increasing the number of MUs in the target social group is capable of reducing the average individual user-delay by $\mathbf{4 4 . 1 \%}$ relying on our scheme. Furthermore, our scheme is more suitable for disseminating a popular piece of content.
\end{abstract}

Index Terms - Content dissemination, content popularity, factor of altruism, pure-birth based Markov chain, delay analysis.

\section{INTRODUCTION}

\section{A. Background and Related Works}

A $\mathrm{S}$ a combination of social science and mobile networks, mobile social networks (MSNs) [1] are attracting an increasing attention across the research community. In the context of MSNs, mobile users (MUs) may form a social group in order to cooperatively disseminate the content of common interest. There are substantial contributions to the performance analysis of epidemic forwarding [2] in mobile ad hoc networks (MANETs). In the context of MANETs, a two-dimensional continuous time Markov chain (CTMC) was proposed in [3] for evaluating the performance of a heterogeneous MANETs. To a further advance, the authors of [4] derived a tight upper bound of the flooding time, which is defined as the number of timesteps required for broadcasting a message from a source node to

Manuscript received April 12, 2015; revised October 18, 2015 and February 10, 2016; accepted February 22, 2016. This work was supported by the RCUK's India-UK Advanced Technology Centre (IU-ATC), that of the EU's concerto project and of the China Scholarship Council (CSC). The work of L. Hanzo was supported by the European Research Council under its Advanced Fellow Award. The associate editor coordinating the review of this paper and approving it for publication was B. Liang.

J. Hu is with the School of Communication and Information Engineering, University of Electronic Science and Technology of China, Chengdu 611731, China (e-mail: hujienavy@outlook.com).

L.-L. Yang and L. Hanzo are with the School of Electronics and Computer Science, University of Southampton, Southampton SO17 1BJ, U.K. (e-mail: 1ly@ecs.soton.ac.uk; 1h@ecs.soton.ac.uk).

Color versions of one or more of the figures in this paper are available online at http://ieeexplore.ieee.org.

Digital Object Identifier 10.1109/TCOMM.2016.2535330 all nodes. Furthermore, in [5] the end-to-end message delivery delay using an epidemic forwarding protocol was investigated theoretically in a composite twin-layer network, which includes a physical MANET and a virtual social network.

However, epidemic forwarding [6] is often criticised as being an end-to-end routing protocol, because it consumes substantial resources of the intermediate nodes, which might not be interested in the information to be relayed. However, if MUs can form a social group and request the content of common interest together, epidemic forwarding becomes an efficient way of cooperatively disseminating the content in the target social group $^{1}$. Content dissemination in purely distributed opportunistic networks was investigated in [7] and [8]. Epidemic forwarding aided content dissemination was invoked in [7], where the users share any content updates with others that they meet in order to improve the coverage quality and to increase the capacity. A socially-aware content placement algorithm was proposed in [8] for enhancing the opportunity of MUs to gain access to their contents of interest.

Some researches focused on a hybrid content dissemination approach. In [9] and [10], the authors investigated how the content providers and network operators can interact for the sake of efficiently distributing the contents with the aid of a coalition game. At the time of writing, epidemic forwarding aided content dissemination is widely studied for the sake of offloading tele-traffic from cellular networks. In [11], the authors proposed a framework for initial content-receiver selection in order to disseminate the content of common interest to as many subscribers as possible before interest in the content subsides. In [12], where MUs were categorised into "helpers" and "subscribers", several algorithms were designed for solving the optimisation problem of offloading multiple types of contents from the cellular networks.

The above-mentioned contributions [2]-[12] focused their attention on user-encounter-based MANETs or 'large-scale MSNs', where the mobile nodes are sparsely distributed across a large area. Typically a rudimentary physical layer model is assumed, namely that if a pair of nodes enter each other's transmission range, the packet can be successfully delivered from the source to the target. Hence, the delivery delay is dominated by the inter-contact duration ${ }^{2}$ of mobile nodes [15], rather than by the wireless signal propagation. Due to

\footnotetext{
${ }^{1}$ Other MUs that do not belong to the target social group are not relied upon for assisting the content dissemination process.

${ }^{2}$ In these treatises, the inter-contact duration of MUs is commonly assumed to obey an exponential distribution, which is demonstrated in [13] with the aid of artificial or synthetic mobility models and in [14] by realistic measured mobility traces.
} 
the underlying long inter-contact duration of the MUs, this user-encounter-based content dissemination is only capable of delivering delay-tolerant services in a large-scale area. As a result, the contributions of [2]-[12] belong to the category of delay-tolerant networks (DTNs). However, typically idealised simplifying assumptions are used in the literature of the DTN paradigm:

- The commonly assumed simplified physical layer model ignores the impact of transmit power, of the path-loss and of the multipath fading, etc.

- The cooperative user-encounter based content dissemination in DTNs is not suitable for delivering delay-sensitive services.

\section{B. Motivations and Contributions}

The conventional method of disseminating the delaysensitive content of common interest relies on BS-aided multicast, where the BS is the sole transmitter. Since the BS-aided multicast has to guarantee the quality of service (QoS) at every content requester, the capacity of multicast channels is predetermined by the worst channel amongst those connecting the BS to the content requesters. In this case, due to the time-variant nature of wireless channels, when the BS multicasts a packet, some MUs may receive it earlier than their less fortunate counterparts. Then, the successful receivers have to remain silent, because the BS would not multicast the second packet, before all the MUs successfully receive the current one.

In high-user-density scenarios, the MUs often share common interest in delay-sensitive content. For instance, the crowd participating in the inauguration of the new Pope share common interest in close-up video-clips of the Pope on the podium. Similarly, supporters in a football stadium share common interest in video-clips of a spectacular goal from different angles or in the score updates from another stadium, as exemplified by Fig. 1. However, the conventional BS-aided multicast is an inefficient technique of disseminating the delay-sensitive content of common interest in these typical densely populated scenarios. The reason for this is two-fold:

- As the content requesters' density increases, the worst channel amongst those connecting the BS and the content requesters becomes even worse, which results in excessive dissemination delay [16].

- Since the dissemination delay is increased, the BS is engaged in multicasting for a longer period, which further delays all other services.

If local MUs form a social group for requesting the content of common interest from the BS together, local communications amongst MUs can be exploited for cooperatively multicasting the packets from the packet owners to the hitherto unserved MUs in the target social group ${ }^{3}$. The potential performance gain of this social group multicast aided content dissemination over the conventional BS-aided multicast arises from the following two benefits:

\footnotetext{
${ }^{3}$ A similar methodology of improving BS-aided multicast was also advocated in [17], which was mainly focused on the selection of the initial receivers However, the authors of [17] have not analysed the content dissemination stage.
}
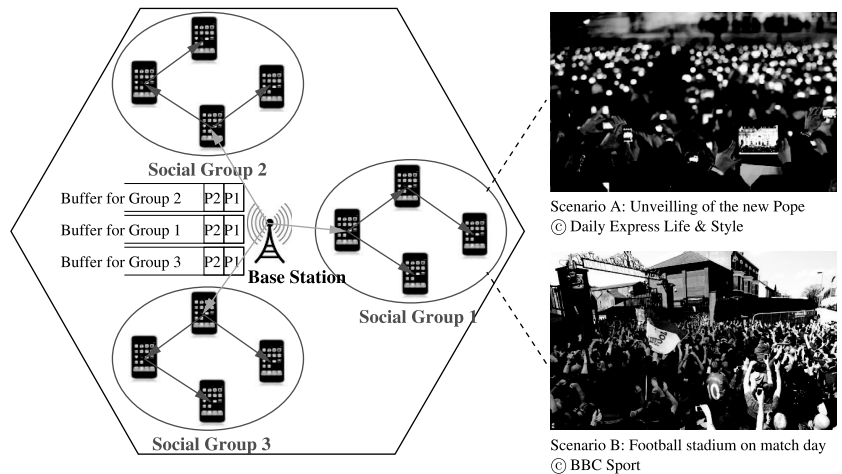

Fig. 1. Social group multicast aided content dissemination in cellular systems.

- Relying on the cooperative multicast of the multiple 134 packet owners results in rich cooperative diversity gains, 135 which in turn improves the packet delivery performance. 136

- Activating direct transmissions amongst the MUs is capa- 137 ble of reducing the distance between a transmitter and 138 receiver pair, which in turn reduces the path-loss-induced 139 channel attenuation between them.

Furthermore, since we offload the content dissemination task 141 from the BS-aided multicast to the local communications 142 amongst the social group members, the BS becomes capable of 143 satisfying other communication demands, which consequently 144 improves the efficiency of the BS's exploitation.

The size of the area covered by a social group should be carefully designed for different scenarios. If the area is as large as a macro-cell, cooperative user-encounter based communication amongst MUs is only suitable for disseminating delay-tolerant information, as we argued at the end of Section I-A. The best option for disseminating delay-sensitive information across a large area is that of classic BS-aided multicast. By contrast, if the area is relatively small, such as a circular area with a radius shorter than a hundred meters, which is comparable to the default transmission range of a $\mathrm{MU}^{4}$, communication efficiency between a transmitter and receiver pair is dominated by the wireless signal propagation properties, rather than by their inter-contact duration. Hence, social group aided cooperative multicast is capable of significantly reducing the delay of the conventional BS-aided multicast, as we emphasized at the beginning of Section I-B. This scenario is termed as a "small-scale MSN" [15], where the channel attenuation factors dominate the associated delay characteristics [19]. Against this background, our novel contributions are as follows:

- A hybrid content dissemination approach is proposed which relies both on BS-aided multicast [20] and on social group multicast aided content dissemination. This process is modelled by a pure-birth based Markov chain $(P B M C)$. Various factors that might affect the performance of the content dissemination are accounted for, including the path-loss-induced channel attenuation, the multipath fading and the users' altruistic versus selfish behaviours, which distinguishes our work from the existing literature of DTNs.

${ }^{4} \mathrm{New}$ Wi-Fi protocols, such as $802.11 \mathrm{n} / \mathrm{ac}$ [18], are capable of supporting a transmission range of hundreds of meters. 
- We model the popularity of different pieces of contents by a Zipf distribution, which affects the specific formation of a social group and hence influences the dissemination process of the content of common interest across the target social group.

- Considering a specific packet of the content of common interest, we analyse the statistical properties of the dissemination delay, which is the time from the BS's instant of multicasting a packet until all the MUs in the target social group receive this packet. We also analyse the individual user-delay, which is the time spanning from the BS multicasting a packet until a specific MU receives this packet.

- The advantages of our social group multicast aided content dissemination scheme over the conventional BSaided multicast are demonstrated by the mobility traces extracted from a realistic subway station scenario.

Note that improving the network infrastructure in high-userdensity areas can certainly enhance the general communication experience of MUs, when supporting phone calls, texts, emails and basic data services. However, it may constitute an inefficient technique of disseminating the content of common interest. It may also be an unwise investment for the network operators, since people often temporarily get together for attending social events. Hence, improving the infrastructure capacity may be wasteful. By contrast, our social group multicast scheme constitutes a more economical and flexible solution for disseminating the content of common interest amongst the social group members, which is based on direct communications between the social group members. We will demonstrate that our social group multicast aided scheme outperforms the BS-aided multicast in terms of disseminating the popular content of common interest in high-user-density areas.

The rest of the paper is organized as follows. In Section II, our system model is introduced. In Section III, we analyse the delay metrics. Furthermore, the exact closed-form formulas are derived for two special cases in Section IV. Our numerical results are provided in Section V. Finally, we conclude in Section VI.

\section{SySTEM OVERVIEW}

Similar to the BS-controlled device-to-device communication services of the LTE network [21], our system operates by obeying a centralised-control regime combined with a decentralised-transmission paradigm ${ }^{5}$, where the BS acts as a centralised controller in order to support the functions of synchronisation ${ }^{6}$, of social group formation as well as of coordination and resource allocation for multiple content owners etc. By contrast, the information transmission is carried out by direct communications between a transmitter and receiver pair.

\footnotetext{
${ }^{5}$ This paradigm has been considered as a part of the forthcoming ' $5 \mathrm{G}$ ' regime, known as the 'LTE-Assisted Wi-Fi Direct' technique [22], where the control signalling exchange is carried out by the LTE-based BS, while the information transmission is realised by the Wi-Fi-based direct communication between a transmitter and receiver pair.

${ }^{6}$ Since the MUs in the cellular system rely on regular control signalling exchange with associated BSs, they can readily synchronise with associated BSs and hence also with each other.
}

TABLE I

The Request Probabilities of $\mathcal{M}=10$ Ranked Popular Contents FOR BOTH $\alpha=0.56$ [24] AND $\alpha=1.0$ [23]

\begin{tabular}{|c|c|c|c|c|c|}
\hline Content & $\mathcal{C}_{1}$ & $\mathcal{C}_{2}$ & $\mathcal{C}_{3}$ & $\mathcal{C}_{4}$ & $\mathcal{C}_{5}$ \\
\hline$\alpha=0.56$ & $21.4 \%$ & $14.6 \%$ & $11.6 \%$ & $9.9 \%$ & $8.7 \%$ \\
\hline$\alpha=1.0$ & $34.1 \%$ & $17.1 \%$ & $11.4 \%$ & $8.5 \%$ & $6.8 \%$ \\
\hline \hline Content & $\mathcal{C}_{6}$ & $\mathcal{C}_{7}$ & $\mathcal{C}_{8}$ & $\mathcal{C}_{9}$ & $\mathcal{C}_{1} 0$ \\
\hline$\alpha=0.56$ & $7.9 \%$ & $7.2 \%$ & $6.7 \%$ & $6.3 \%$ & $6.0 \%$ \\
\hline$\alpha=1.0$ & $5.7 \%$ & $4.9 \%$ & $4.3 \%$ & $3.8 \%$ & $3.4 \%$ \\
\hline
\end{tabular}

\section{A. Content Popularity and Social Group Formation}

The interest of a MU in a specific piece of content $\mathcal{C}_{i}$ may 225 be modelled by the probability $\operatorname{Pr}\left(\mathcal{C}_{i}\right)$ of this $\mathrm{MU}$ requesting $\mathcal{C}_{i} 226$ from the BS. Having a higher request probability $\operatorname{Pr}\left(\mathcal{C}_{i}\right)$ indi- 227 cates that the MU is more interested in the content $\mathcal{C}_{i}$. The 228 statistical analysis of the realistic video viewing behaviours 229 exhibited by YouTube users revealed that a small fraction of 230 popular contents attract the interest of a large fraction of users 231 [23], [24]. Furthermore, the request probabilities of a set of 232 ranked contents, say $\left\{\mathcal{C}_{i} \mid i=1, \ldots, \mathcal{N}\right\}$, may be modelled by 233 a Zipf distribution [25], [26]. Here $\mathcal{M}$ is the number of contents 234 studied and the subscript $i$ represents the particular position 235 of $\mathcal{C}_{i}$ in the popularity list. A smaller integer subscript $i$ indi- 236 cates that the content is more popular and hence it is likely to 237 be requested more frequently. Therefore, the probability of the 238 piece of content $\mathcal{C}_{i}$ being requested is expressed as

$$
\operatorname{Pr}\left(\mathfrak{C}_{i}\right)=\frac{\frac{1}{i^{\alpha}}}{\sum_{j=1}^{\mathcal{M}} \frac{1}{j^{\alpha}}},
$$

where $\alpha$ is a predefined exponent. Having a higher value of 240 $\alpha$ results in more intense interests in the top-ranked pieces of 241 contents, as shown in TABLE I.

Assuming that we have $\mathcal{N}$ MUs within the area studied, these 243 MUs independently request one piece of contents from the set 244 $\left\{\mathcal{C}_{i} \mid i=1, \ldots, \mathcal{M}\right\}$ with the corresponding probability defined 245 in (1). The MUs requesting the same content $\mathcal{C}_{i}$ form a social 246 group $\mathcal{G}_{i}$ in order to cooperatively disseminate the content of 247 common interest across the social group. Hence, the size of 248 the social group $\mathcal{G}_{i}$ requesting the same content $\mathcal{C}_{i}$ obeys a 249 Binomial distribution, which is denoted as $\left|\mathcal{G}_{i}\right| \sim B\left[\mathcal{N}, \operatorname{Pr}\left(\mathcal{C}_{i}\right)\right] .250$ In order to exclude the case of $|\mathcal{G}|=0$, we adjust the probability 251 mass function (pmf) of $\left|\mathcal{G}_{i}\right|$, which is expressed as

$$
\operatorname{Pr}\left(\left|\mathcal{G}_{i}\right|=N\right)=\frac{\left(\begin{array}{l}
\mathcal{N} \\
N
\end{array}\right)\left[\operatorname{Pr}\left(\mathcal{C}_{i}\right)\right]^{N}\left[1-\operatorname{Pr}\left(\mathcal{C}_{i}\right)\right]^{\mathcal{N}-N}}{1-\left[1-\operatorname{Pr}\left(\mathcal{C}_{i}\right)\right]^{\mathcal{N}}} .
$$

where $N$ is the specific size of the social group $\mathcal{G}_{i}$. As a result, 253 the average $\overline{\mathcal{P}}\left(\mathcal{C}_{i}\right)$ of a specific delay metric associated with dis- 254 seminating the content $\mathcal{C}_{i}$ across the social group $\mathcal{G}_{i}$, whose size 255 is an adjusted-Binomially distributed random variable, can be 256 expressed as

$$
\overline{\mathcal{P}}\left(\mathfrak{C}_{i}\right)=\sum_{N=1}^{\mathcal{N}} \mathcal{P}\left(\left|\mathcal{G}_{i}\right|=N\right) \cdot \operatorname{Pr}\left(\left|\mathcal{G}_{i}\right|=N\right),
$$

\footnotetext{
${ }^{7}$ If no MUs requests the content, we do not have to study the content dissemination performance.
} 
where $\mathcal{P}\left(\left|\mathcal{G}_{i}\right|=N\right)$ is a delay metric, which is a function of the deterministic social group size $\left|\mathcal{G}_{i}\right|=N$. Given the social group size $N$, in Section III, we will derive various delay metrics that can replace $\mathcal{P}\left(\left|\mathcal{G}_{i}\right|=N\right)$ in (3) in order to evaluate the impact of content popularity on the content dissemination performance.

To sum up, we assume that $N$ MUs form a social group in order to request the content of common interest from a BS, as shown in Fig. 1. The formation of a social group depends on the following conditions:

- MUs share the same interest in a given piece of content;

- The content of common interest is of delay-sensitive nature;

- MUs roam in a bounded area having a relatively small size and they are geographically close to each other.

\section{B. Network Layer}

In order to disseminate the content of common interest across a social group, the BS creates a specific queue for buffering all the packets of the requested content and prepares for disseminating these packets one by one, as described below.

Firstly, the BSs are employed for repeatedly multicasting the packet currently at the head of the buffer, until at least one of the MUs in the target social group successfully receives it. Then, this packet is cooperatively disseminated across the social group using multicast techniques.

During the dissemination process, after successfully receiving the packet, the packet owners (POs) may make their decisions independently as to whether they would or would not forward the packet during the following stage of the dissemination, as shown in Fig. 2. Once some POs decided to further forward the packet, they would repeatedly multicast it until at least one unserved MU in the target social group successfully receives it. Afterwards, the new POs join the original PO set. Both the new POs and the original POs make new packet forwarding decisions again for the subsequent stage of dissemination. The probability of a PO willing to forward the packet is denoted as $q(0 \leq q \leq 1)$, which is termed as the Factor of Altruism (FA). At a given instant, there might not be any POs willing to further forward the packet. As a result, the unserved MUs in the target social group have to receive the packet directly from the BS. Similarly, the BS repeatedly multicasts the packet until at least one unserved MU in the target social group receives it.

During the content dissemination process, similar to the conventional BS-aided multicast, the BS keeps a specific packet at the head of the buffer, until all the MUs in the target social group successfully receive it. Then the packet is dropped from the buffer and the BS is ready to disseminate the subsequent one.

\section{Physical (PHY) Layer}

In the PHY layer, the radio propagation between any pair of transmitter and receiver is assumed to experience uncorrelated stationary Rayleigh flat-fading. Hence, the square of the fading amplitudes $\left|h_{l}(t)\right|^{2}$ during the $t^{\text {th }}$ time slot (TS) obeys an exponential distribution having a unity mean, whose tail distribution

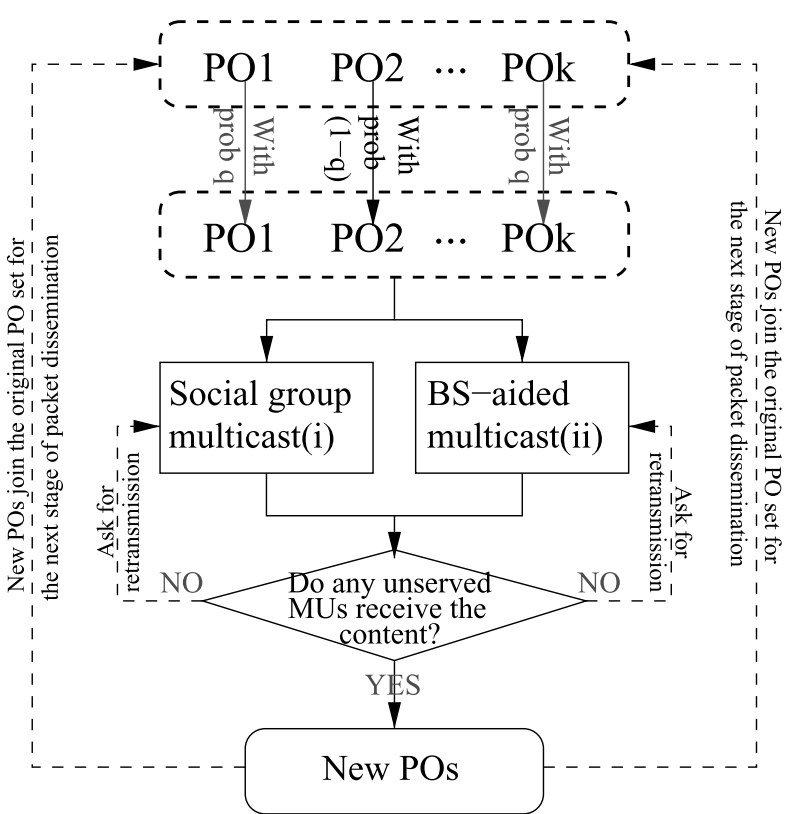

(i): Some POs are willing to further forward the content of common interest. (ii): None POs are willing to further forward the content of common interest.

Fig. 2. Actions of POs during the spontaneous content dissemination.

function $(\operatorname{tdf})$ is $\operatorname{Pr}\left[\left|h_{l}(t)\right|^{2}>x\right]=\mathrm{e}^{-x}$. Given an arbitrary 312 distance $y_{l}$ in meters, the path loss (PL) $\Omega_{l}$ is expressed as [27]: 313

$$
\Omega_{l}\left(y_{l}\right)= \begin{cases}1, & y_{l}<d_{0}, \\ \left(\frac{4 \pi f_{c}}{c}\right)^{\kappa} y_{l}^{\kappa}, & y_{l} \geq d_{0},\end{cases}
$$

where $c$ is the speed of light and $f_{c}$ is the carrier frequency, 314 whereas $\kappa$ is the PL exponent and $d_{0}$ is the distance from the 315 transmitter to the 'near-field' edge.

The random distance $Y_{l}$ is determined by the mobility pattern 317 of the MUs in the target social group. The following mobility 318 model is invoked for our performance analysis:

Definition 1 (Uniform mobility model): The position of the 320 $i^{\text {th }}$ MU during the $t^{\text {th }}$ time interval is denoted by $\mathbf{P}_{i}(t)$, which 321 obeys a stationary and ergodic process having a uniform dis- 322 tribution in the area considered. Moreover, the positions of 323 different MUs are independently and identically distributed 324 (i.i.d.).

This mobility model has been widely adopted for the per- 326 formance analysis of MANETs [28], [29]. Let the probability 327 density function (pdf) of the random distance $Y_{l}$ between any 328 two MUs be denoted by $f_{Y_{l}}\left(y_{l}\right)$. Our forthcoming performance 329 analysis is applicable not only to the uniform mobility model, 330 but to any arbitrary mobility model.

Note that, the index $l$ in the formulas is a generic subscript, 332 which represents ' $b$ ' when the BS is the transmitter, while it 333 represents ' $s$ ' when a MU is the transmitter. In the rest of the 334 paper, ' $l$ ', ' $b$ ' and ' $s$ ' hold the same meaning.

\section{Medium-Access-Control (MAC) Layer}

During a TS, a packet of the content is assumed to be suc- 337 cessfully received by a MU, provided that the instantaneous 338 
received signal-to-noise-ratio (SNR) is higher than a predefined threshold $\gamma$ [30]. In order to avoid collisions amongst multiple transmitters, orthogonal-frequency-divisionmultiple-access (OFDMA) or code-division-multiple-access (CDMA) may be invoked for allocating each transmitter an orthogonal channel. We denote the successful packet reception probability (SPRP) of a link as $\mu_{l}\left(y_{l}\right)$. By jointly considering the PHY layer model, the SPRP is derived as

$$
\begin{aligned}
\mu_{l}\left(y_{l}\right) & =\operatorname{Pr}\left(\frac{\operatorname{Pr}_{l}^{t x}\left|h_{l}(t)\right|^{2}}{\Omega_{l}\left(y_{l}\right) N_{0} W_{l}}>\gamma\right) \\
& = \begin{cases}\mathrm{e}^{-\frac{\gamma N_{0} W_{l}}{\operatorname{Pr}_{l}^{t x}}}, & y_{l}<d_{0}, \\
\mathrm{e}^{-\frac{\gamma N_{0} W_{l}}{\operatorname{Pr}_{l}^{t x}}\left(\frac{4 \pi f_{c}}{c}\right)^{\kappa} y_{l}^{\kappa}}, & y_{l} \geq d_{0},\end{cases}
\end{aligned}
$$

where $P_{l}^{t x}$ is the corresponding transmit power and $N_{0} W_{l}$ is the noise power in a communication bandwidth $W_{l}$. Given the pdf $f_{Y_{l}}\left(y_{l}\right)$ of the random distance $Y_{l}$, the average SPRP $\bar{\mu}_{l}$ of a link is derived as

$$
\begin{aligned}
\bar{\mu}_{l}= & \int_{0}^{d_{0}} \mathrm{e}^{-\frac{\gamma N_{0} W_{l}}{P_{l}^{t x}}} f_{Y_{l}}\left(y_{l}\right) d y_{l} \\
& +\int_{y_{l} \geq d_{0}} \mathrm{e}^{-\frac{\gamma N_{0} W_{l}}{P_{l}^{t x}}\left(\frac{4 \pi f_{c}}{c}\right)^{\kappa} y^{\kappa}} f_{Y_{l}}\left(y_{l}\right) d y_{l}
\end{aligned}
$$

Substituting the corresponding parameters and the pdf of the random distance into (6), we can obtain the average SPRP $\bar{\mu}_{s}$ between a pair of MUs and $\bar{\mu}_{b}$ between the BS and a MU. Moreover, the following lemma is proposed for our further analysis:

Lemma 1: Given the average SPRP $\bar{\mu}_{l}$ of a link during a TS, the average SPRP during a sufficiently short time interval $\Delta t$ ( $\Delta t \ll 1 \mathrm{TS}$ ) is approximately $\bar{\mu}_{l} \Delta t$.

Proof: The proof can be found in Appendix A.

Note that the SPRP also represents the normalized throughput, whose unit is packet/TS [30]. In more details, $\bar{\mu}_{l}$ indicates that $\bar{\mu}_{l}$ packets in average can be successfully received during a TS. Therefore, during $\Delta t(\leq 1) \mathrm{TS}$, only $\bar{\mu}_{l} \Delta t$ packets in average can be successfully received.

\section{Delay AnAlysis of the PaCKet Dissemination}

In this section, various delay metrics of the packet dissemination process are derived with respect to a specific group size $N$. These metrics may replace the performance function $\mathcal{P}\left(\left|\mathcal{G}_{i}\right|=\right.$ $N$ ) in (3) in order to characterize the average performance as a function of the content popularity.

\section{A. Pure Birth Markov Chain (PBMC)}

Let us assume that there are $N$ MUs in a considered social group. During the process of packet dissemination across the target social group, the number of POs steadily increases until all the $N$ social group members successfully receive the packet of common interest. Hence, the packet dissemination process can be modelled by a discrete-time PBMC having $(N+1)$ states, as shown in Fig. 3. In this PBMC, the states represent

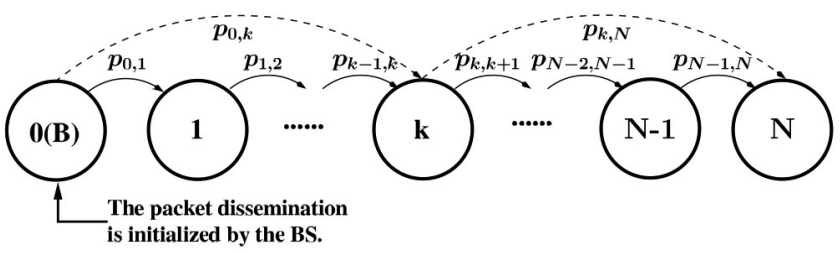

Fig. 3. A pure-birth Markov chain having an absorption state.

the corresponding numbers of POs having received the packet. 379 State transition only occurs from a lower-indexed state to a 380 higher-indexed one. Specifically, the state transition emerges 381 from state 0 , which represents the initial stage of the BS-aided 382 multicast, and terminates in state $N$, which indicates that all 383 the $N$ MUs in the target social group have received the desired 384 packet.

385

Let us first consider the general transition probability from 386 state $k$ to state $(k+m)$, where we have $1 \leq k \leq(N-1)$ and 387 $0 \leq m \leq(N-k)$. In the light of the selfish user-behaviour 388 considered, we assume that only $n_{k}, 1 \leq n_{k} \leq k$, POs are 389 willing to further disseminate the packet at the current stage. 390 Therefore, any unserved MU out of the $(N-k)$ unserved ones 391 is connected to the $n_{k}$ POs by $n_{k}$ wireless links, and any of 392 these links has the probability of $\bar{\mu}_{s} \Delta t$ to successfully deliver 393 the packet during the time interval $\Delta t$ according to Lemma 1. 394 As a result, given that $n_{k}$ POs independently deliver their pack- 395 ets to the same target, the SPRP of an unserved MU is expressed 396 as $\left[1-\left(1-\bar{\mu}_{s} \Delta t\right)^{n_{k}}\right]$. Furthermore, the state transition prob- 397

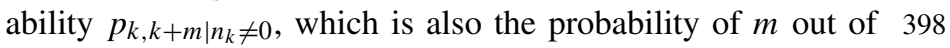
the $(N-k)$ unserved MUs successfully receiving the packet 399 during the current time interval $\Delta t$, can be expressed as

$$
\begin{aligned}
p_{k, k+m \mid n_{k} \neq 0}= & \left(\begin{array}{c}
N-k \\
m
\end{array}\right)\left[1-\left(1-\bar{\mu}_{s} \Delta t\right)^{n_{k}}\right]^{m} \\
& .\left(1-\bar{\mu}_{s} \Delta t\right)^{n_{k}(N-k-m)} \\
= & \left(\begin{array}{c}
N-k \\
m
\end{array}\right)\left[1-\sum_{i=0}^{n_{k}}\left(\begin{array}{c}
n_{k} \\
i
\end{array}\right)\left(-\bar{\mu}_{s} \Delta t\right)^{i}\right]^{m} \\
& .\left(1-\bar{\mu}_{s} \Delta t\right)^{n_{k}(N-k-m)} \\
= & \left(\begin{array}{c}
N-k \\
m
\end{array}\right)\left[\sum_{i=1}^{n_{k}}\left(\begin{array}{c}
n_{k} \\
i
\end{array}\right)(-1)^{i+1}\left(\bar{\mu}_{s} \Delta t\right)^{i}\right]^{m} \\
& .\left(1-\bar{\mu}_{s} \Delta t\right)^{n_{k}(N-k-m) .}
\end{aligned}
$$

According to (7), the state transition probability $p_{k, k+m \mid n_{k} \neq 0} 401$ has the same growth rate as $\bar{\mu}_{s}^{m} \Delta t^{m}$. Hence, the adjacent-state 402 transition probability $p_{k, k+1 \mid n_{k} \neq 0}$ of traversing from state $k$ to 403 state $(k+1)$ has the same growth rate as $\bar{\mu}_{s} \Delta t$. Substituting 404 $m=1$ into (7), $p_{k, k+1 \mid n_{k} \neq 0}$ can be expressed as

$$
\begin{aligned}
p_{k, k+1 \mid n_{k} \neq 0} & =(N-k) n_{k} \bar{\mu}_{s} \Delta t \\
+(N-k) & \sum_{i=2}^{n_{k}(N-k-1)}\left(\begin{array}{c}
n_{k}(N-k-1) \\
i
\end{array}\right)\left(-\bar{\mu}_{s} \Delta t\right)^{i} \\
& \left.-\sum_{j=2}^{n_{k}(N-k)}\left(\begin{array}{c}
n_{k}(N-k) \\
j
\end{array}\right)\left(-\bar{\mu}_{s} \Delta t\right)^{j}\right] .
\end{aligned}
$$


TABLE II

PARAMETERS OF THE PHY LAYER

\begin{tabular}{|l|l|l|}
\hline & BS to MUs & MUs to MUs \\
\hline Transmit Power & $P_{b}^{t x}=31 \mathrm{dBm}$ & $P_{s}^{t x}=0 \sim 10 \mathrm{dBm}$ \\
\hline Carrier Freq & $f_{c, b}=1.8 \mathrm{GHz}$ & $f_{c, s}=2.4 \mathrm{GHz}$ \\
\hline Bandwidth & $W_{b}=10 \mathrm{MHz}$ & $W_{s}=10 \mathrm{MHz}$ \\
\hline Noise PSD & \multicolumn{2}{|c|}{$N_{0}=-174 \mathrm{dBm} / \mathrm{Hz}\left(20^{\circ} C\right)$} \\
\hline SNR Threshold & \multicolumn{2}{|c|}{$\gamma=10 \mathrm{~dB}$} \\
\hline PL Parameters & Exponent: $\kappa=3 ;$ Ref Distance: $d_{0}=1 \mathrm{~m}$ \\
\hline
\end{tabular}
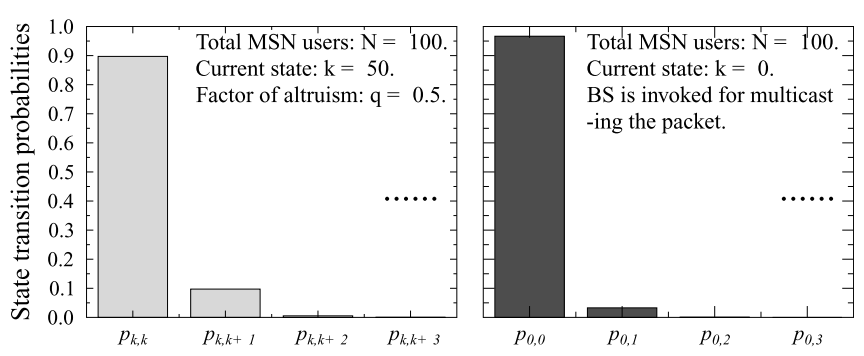

Fig. 4. State transition probabilities when $\Delta t=0.001 \mathrm{TS}$.

The terms in the square brackets of (8) have the same growth rate as $\bar{\mu}_{s}^{2} \Delta t^{2}$. Compared to the first term $(N-k) n_{k} \bar{\mu}_{s} \Delta t$ in (8), the terms in the square brackets are negligibly low, when $\bar{\mu}_{s} \Delta t$ is close to zero. Hence, in this case, we can approximate $p_{k, k+1 \mid n_{k} \neq 0}$ as $p_{k, k+1 \mid n_{k} \neq 0} \approx(N-k) n_{k} \bar{\mu}_{s} \Delta t$. Similarly, when $\bar{\mu}_{s} \Delta t$ is close to zero, $p_{k, k+m \mid n_{k} \neq 0}$ associated with $m \geq$ 2 in (7) can be approximated as $p_{k, k+m \mid n_{k} \neq 0} \approx 0$. Moreover, substituting $m=0$ into (7), we obtain the probability of the PBMC sojourning in the current state $k$ after the time interval $\Delta t$, which is $p_{k, k \mid n_{k} \neq 0}=\left(1-\bar{\mu}_{s} \Delta t\right)^{n_{k}(N-k)}$. Again, when $\bar{\mu}_{s} \Delta t$ is very close to zero, $p_{k, k \mid n_{k} \neq 0}$ can be approximated as $p_{k, k \mid n_{k} \neq 0} \approx 1-n_{k}(N-k) \bar{\mu}_{s} \Delta t$.

Another scenario is that no POs are willing to forward the packet, corresponding to the case $n_{k}=0$. Then the $(N-k)$ unserved MUs have to receive the packet directly from the BS. Similarly, we can also demonstrate that $p_{k, k+1 \mid n_{k}=0} \approx(N-k) \bar{\mu}_{b} \Delta t$ and $p_{k, k \mid n_{k}=0} \approx 1-(N-$ $k) \bar{\mu}_{b} \Delta t$, while $p_{k, k+m \mid n_{k}=0} \approx 0$ for $m \geq 2$, provided that $\bar{\mu}_{b} \Delta t$ is sufficiently small. Furthermore, it can be shown that $p_{0,1} \approx$ $N \bar{\mu}_{b} \Delta t, p_{0,0} \approx 1-N \bar{\mu}_{b} \Delta t$ and $p_{0, m} \approx 0$ for $m \geq 2$, provided that $\bar{\mu}_{b} \Delta t$ is sufficiently small.

According to the PHY layer parameters in TABLE II, we plot the state transition probabilities for state $k=50$ and for state $k=0$, respectively, in Fig. 4 . We observe from Fig. 4 that the state transition probabilities of $p_{k, k+m}$ and $p_{0, m}$ for $m \geq 2$ are negligibly low, which demonstrates the high accuracy of the above approximations involved.

Therefore, assuming a sufficiently short time interval $\Delta t$, only adjacent-state transitions occur during the process modelled by the discrete-time PBMC, as shown in Fig. 3.

\section{B. Delay of State Transition}

In order to study the delay statistics of disseminating a specific packet, we need to know the specific delay that the PBMC spends in a particular state, which is termed as the state transition delay. As a result, the following lemma may 440 be formulated:

Lemma 2: Given the state transition probability $\tilde{\mu}_{k} \Delta t$ from 442 the current state $k$ to state $(k+1)$, the transition delay from 443 state $k$ to state $(k+1)$ obeys the exponential distribution with a 444 mean of $1 / \tilde{\mu}_{k}$ TS, provided that $\Delta t$ is sufficiently small. Here, 445 $\tilde{\mu}_{k}$ is termed as the transition rate. 446

Proof: The proof can be found in Appendix B. 447

Based on Lemma 2, the discrete-time PBMC seen in Fig. 3448 can be further simplified to a continuous-time PBMC, which 449 only has adjacent-state transitions. The transition rate of this 450 continuous-time PBMC can be shown to be $p_{k, k+1} / \Delta t$, where 451 $p_{k, k+1}$ is the adjacent-state transition probability derived in 452 Section III-A.

Let us first consider the delay $T_{k}$ of the transition from state 454 $k$ to $(k+1)$, when $k \geq 1$. Since each PO has a probability $q$ of 455 forwarding the packet, in the current state $k$, the number $n_{k}(0 \leq 456$ $\left.n_{k} \leq k\right)$ of POs willing to forward the packet obeys a Binomial 457 distribution having a pair of parameters $k$ and $q$, whose pmf is 458 given by [31]

$$
p\left(n_{k}\right)=\left(\begin{array}{c}
k \\
n_{k}
\end{array}\right) q^{n_{k}}(1-q)^{k-n_{k}}, \quad n_{k}=0,1, \ldots, k
$$

For the case of $n_{k} \neq 0$, we have $p_{k, k+1 \mid n_{k} \neq 0} \approx n_{k}(N-460$ $k) \bar{\mu}_{s} \Delta t$. According to Lemma 2 , the delay $T_{k}$ of the transition 461 from state $k$ to state $(k+1)$ obeys an exponential distribu- 462 tion having a rate of $n_{k}(N-k) \bar{\mu}_{s}=n_{k} \mu_{s, k}$, where $\mu_{s, k}=463$ $(N-k) \bar{\mu}_{s}$. Hence, when $n_{k} \neq 0$, the conditional pdf, the mean 464 and the second moment of $T_{k}$ may be formulated as

$$
\begin{aligned}
f_{T_{k} \mid n_{k}}\left(t_{k}\right) & =n_{k} \mu_{s, k} \cdot \mathrm{e}^{-n_{k} \mu_{s, k} t_{k}}, t_{k} \geq 0 \\
\mathcal{E}\left[T_{k} \mid n_{k}\right] & =\int_{0}^{\infty} t_{k} f_{T_{k} \mid n_{k}}\left(t_{k}\right) d t_{k}=\frac{1}{n_{k} \mu_{s, k}}, \\
\mathcal{E}\left[T_{k}^{2} \mid n_{k}\right] & =\int_{0}^{\infty} t_{k}^{2} f_{T_{k} \mid n_{k}}\left(t_{k}\right) d t_{k}=\frac{2}{\left(n_{k} \mu_{s, k}\right)^{2}} .
\end{aligned}
$$

For the case of $n_{k}=0$, we have $p_{k, k+1 \mid n_{k}=0} \approx(N-466$ $k) \bar{\mu}_{b} \Delta t$, as the MUs in the target social group have to receive 467 the packet from the BS. According to Lemma 2, the delay $T_{k} 468$ of the transition from state $k$ to $(k+1)$ obeys an exponential 469 distribution having a rate of $\mu_{b, k}=(N-k) \bar{\mu}_{b}$. Hence, given 470 $n_{k}=0$, the conditional pdf, the mean and the second moment 471 of $T_{k}$ are derived as

$$
\begin{gathered}
f_{T_{k} \mid n_{k}=0}\left(t_{k}\right)=\mu_{b, k} \cdot \mathrm{e}^{-\mu_{b, k} t_{k}}, t_{k} \geq 0 \\
\mathcal{E}\left[T_{k} \mid n_{k}=0\right]=\int_{0}^{\infty} t_{k} f_{T_{k} \mid n_{k}=0}\left(t_{k}\right) d t_{k}=\frac{1}{\mu_{b, k}}, \\
\mathcal{E}\left[T_{k}^{2} \mid n_{k}=0\right]=\int_{0}^{\infty} t_{k}^{2} f_{T_{k} \mid n_{k}=0}\left(t_{k}\right) d t_{k}=\frac{2}{\mu_{b, k}^{2}} .
\end{gathered}
$$

According to the classic Bayesian principle [31], the pdf of $T_{k} 473$ may be expressed as 


$$
\begin{aligned}
f_{T_{k}}\left(t_{k}\right)= & \sum_{n_{k}=1}^{k} f_{T_{k} \mid n_{k}}\left(t_{k}\right) \cdot p\left(n_{k}\right)+f_{T_{k} \mid n_{k}=0}\left(t_{k}\right) \cdot p\left(n_{k}=0\right) \\
= & \sum_{n_{k}=1}^{k}\left(\begin{array}{c}
k \\
n_{k}
\end{array}\right) q^{n_{k}}(1-q)^{k-n_{k}} \cdot n_{k} \mu_{s, k} \mathrm{e}^{-n_{k} \mu_{s, k} t_{k}} \\
& \quad+(1-q)^{k} \mu_{b, k} \mathrm{e}^{-\mu_{b, k} t_{k}} .
\end{aligned}
$$

Moreover, the mean of $T_{k}$ is formulated as

$$
\begin{aligned}
\mathcal{E}\left[T_{k}\right] & =\mathcal{E}\left[T_{k} \mid n_{k}=0\right] p\left(n_{k}=0\right)+\sum_{n_{k}=1}^{k} \mathcal{E}\left[T_{k} \mid n_{k}\right] p\left(n_{k}\right) \\
& =\underbrace{\frac{(1-q)^{k}}{\mu_{b, k}}}_{\mathcal{E}\left[T_{k, b}\right]}+\underbrace{\sum_{n_{k}=1}^{k}\left(\begin{array}{c}
k \\
n_{k}
\end{array}\right) \frac{q^{n_{k}}(1-q)^{k-n_{k}}}{n_{k} \mu_{s, k}}}_{\mathcal{E}\left[T_{k, s}\right]}
\end{aligned}
$$

where $\mathcal{E}\left[T_{k, b}\right]$ represents the average duration of the BS-aided multicasting invoked during the transition from state $k$ to state $(k+1)$, where $\mathcal{E}\left[T_{k, s}\right]$ is the average duration of the social group multicasting during this state transition. Furthermore, the second moment of $T_{k}$ is formulated as

$$
\begin{aligned}
\mathcal{E}\left[T_{k}^{2}\right] & =\mathcal{E}\left[T_{k}^{2} \mid n_{k}=0\right] p\left(n_{k}=0\right)+\sum_{n_{k}=1}^{k} \mathcal{E}\left[T_{k}^{2} \mid n_{k}\right] p\left(n_{k}\right) \\
& =\frac{2(1-q)^{k}}{\mu_{b, k}^{2}}+\sum_{n_{k}=1}^{k}\left(\begin{array}{c}
k \\
n_{k}
\end{array}\right) \frac{2 q^{n_{k}}(1-q)^{k-n_{k}}}{\left(n_{k} \mu_{s, k}\right)^{2}}
\end{aligned}
$$

From (17) and (18), we can also derive the variance of $T_{k}$ by using the formula of $\operatorname{Var}\left[T_{k}\right]=\mathcal{E}\left[T_{k}^{2}\right]-\left\{\mathcal{E}\left[T_{k}\right]\right\}^{2}$. Furthermore, we may simply derive the pdf, the mean and the second moment of the transition delay $T_{0}$ from state 0 to state 1 by substituting $k=0$ in (13), (14), and (15), respectively.

\section{Dissemination Delay}

Since the delay of the transition from a state to its successor is independent of any other state transition's delay, and given that the dissemination delay across the target social group is

$$
\mathcal{E}\left[T_{D}\right]=\sum_{k=0}^{N-1} \frac{(1-q)^{k}}{\mu_{b, k}}+\sum_{k=1}^{N-1} \sum_{n_{k}=1}^{k}\left(\begin{array}{c}
k \\
n_{k}
\end{array}\right) \frac{q^{n_{k}}(1-q)^{k-n_{k}}}{n_{k} \mu_{s, k}},
$$

while the variance of $T_{D}$ can be formulated as $\operatorname{Var}\left[T_{D}\right]=$ $\sum_{k=0}^{N-1} \operatorname{Var}\left[T_{k}\right]$.

There is no exact closed-form tdf for the dissemination delay $T_{D}$ in this general case. However, given its mean and variance, we may approximate it as a random variable obeying the Gamma distribution, which is usually more accurate than its Gaussian counterpart, when non-negative random variables are concerned [32]. According to the theory of the Gamma distribution [33], it is uniquely and unambiguously described by its shape parameter $m=\left\{E\left[T_{D}\right]\right\}^{2} / \operatorname{Var}\left[T_{D}\right]$ and scale parameter $\Theta=\operatorname{Var}\left[T_{D}\right] / E\left[T_{D}\right]$. Then, given a delay threshold $D_{t h}$, we may derive the approximate probability of the dissemination delay $T_{D}$ exceeding $D_{t h}$ as defined as $T_{D}=\sum_{k=0}^{N-1} T_{k}$, the mean of $T_{D}$ can be expressed as

$$
\operatorname{Pr}\left(T_{D}>D_{t h}\right) \approx \frac{\Gamma\left(m, \frac{D_{t h}}{\Theta}\right)}{\Gamma(m)}=\frac{\Gamma\left(\frac{\left\{E\left[T_{D}\right]\right\}^{2}}{\operatorname{Var}\left[T_{D}\right]}, \frac{D_{t h} E\left[T_{D}\right]}{\operatorname{Var}\left[T_{D}\right]}\right)}{\Gamma\left(\frac{\left\{E\left[T_{D}\right]\right\}^{2}}{\operatorname{Var}\left[T_{D}\right]}\right)} .
$$

The accuracy of (20) will be verified by the Monte-Carlo 504 simulation in Section V.

\section{Individual User-Delay}

A specific MU $\mathcal{A}$ in the target social group may receive the 507 packet at any state spanning from 1 to $N$ during the process 508 of state transitions. When considering the transition from state 509 $(k-1)$ to $k(1 \leq k \leq N)$, any of the $(N-k+1)$ unserved 510 MUs may successfully receive the packet with a probability 511 of $1 /(N-k+1)$, and may not receive it with a probability of 512 $(N-k) /(N-k+1)$. Specifically, the probability of $\mathcal{A}$ receiv- 513 ing the packet in state $k$, which naturally implies that $\mathcal{A}$ has 514 not received the packets at any of the previous states, may be 515 expressed as

$$
p_{k}=\frac{1}{N-k+1} \cdot \prod_{i=1}^{k-1} \frac{N-i}{N-i+1}=\frac{1}{N}, \quad 1 \leq k \leq N .
$$

Hence, given that $\mathcal{A}$ receives the packet in state $k$, the 517 individual user-delay of $\mathcal{A}$ is expressed as $T_{\mathcal{A} \mid k}=\sum_{j=0}^{k-1} T_{k} 518$ and the conditional pdf of $T_{\mathcal{A} \mid k}$ is expressed as $f_{T_{\mathcal{A}} \mid k}\left(t_{\mathcal{A}}\right)=519$ $f_{T_{0}+\cdots+T_{k-1}}\left(t_{\mathcal{A}}\right)$. According to the Bayesian principle [31], the 520 pdf of the individual user-delay $T_{\mathcal{A}}$ can be expressed as:

$$
f_{T_{\mathcal{A}}}\left(t_{\mathcal{A}}\right)=\sum_{k=1}^{N} f_{T_{\mathcal{A}} \mid k}\left(t_{\mathcal{A}}\right) \cdot p_{k}=\sum_{k=1}^{N} \frac{f_{T_{0}+\cdots+T_{k-1}}\left(t_{\mathcal{A}}\right)}{N} .
$$

Furthermore, owing to the fact that $\left\{T_{0}, T_{1}, \ldots, T_{k-1}\right\}$ are inde- 522 pendent of each other, the average of $T_{\mathcal{A}}$ can be obtained 523 as

$$
\begin{aligned}
\mathcal{E}\left[T_{\mathcal{A}}\right] & =\int_{0}^{\infty} t_{\mathcal{A}} \sum_{k=1}^{N} \frac{f_{T_{0}+\cdots+T_{k-1}}\left(t_{\mathcal{A}}\right)}{N} d t_{\mathcal{A}}=\sum_{k=1}^{N} \frac{1}{N} \cdot \sum_{i=0}^{k-1} \mathcal{E}\left[T_{i}\right] \\
& =\sum_{k=1}^{N} \frac{N-k+1}{N} \mathcal{N}\left[T_{k-1}\right]
\end{aligned}
$$

where $\mathcal{E}\left[T_{k-1}\right]$ is given by (17). Furthermore, the second 525 moment of $T_{\mathcal{A}}$ is given by 
where $\boldsymbol{\xi}_{k}=\left(\mathcal{E}\left[T_{0}\right], \mathcal{E}\left[T_{1}\right], \ldots, \mathcal{E}\left[T_{k-1}\right]\right)^{T}, \quad \mathbf{H}_{k}$ is a $k \times k$ matrix, whose elements are all ones, and $\mathbf{I}_{k}$ is a $k \times k$ identity matrix. Consequently, the variance of $T_{\mathcal{A}}$ can be expressed as $\operatorname{Var}\left(T_{\mathcal{A}}\right)=\mathcal{E}\left[T_{\mathcal{A}}^{2}\right]-\left\{\mathcal{E}\left[T_{\mathcal{A}}\right]\right\}^{2}$. Hence, by substituting $\mathcal{E}\left[T_{\mathcal{A}}\right]$ and $\operatorname{Var}\left[T_{\mathcal{A}}\right]$ into (20), we may obtain the approximate probability of $T_{\mathcal{A}}$ exceeding threshold $D_{t h}$.

\section{Delay Metrics for Special CASES}

\section{A. Case 1: Conventional BS-Aided Multicast $(q=0)$}

In this pessimistic case, all the MUs in the target social group are selfish during the packet dissemination process. Hence, the BS has to disseminate the packet to all the MUs in the target social group.

1) Dissemination Delay: When FA is $q=0$, according to Eqs.(13) (15) in Section III-B, the state transition delays $\left\{T_{k}, k=0,1, \ldots,(N-1)\right\}$ are the independent exponentially distributed variables associated with the rates of $\left\{\widetilde{\mu}_{k}=(N-\right.$ $\left.k) \bar{\mu}_{b}, k=0,1, \ldots,(N-1)\right\}$. Since the dissemination delay is defined as $T_{D}=\sum_{k=0}^{N-1} T_{k}, T_{D}$ obeys the hypoexponential distribution [34]. Furthermore, since the rates of $\left\{T_{k}, k=\right.$ $0,1, \ldots,(N-1)\}$ are different from each other, the pdf of $T_{D}$ can be expressed as

$$
f_{T_{D} \mid q=0}\left(t_{D}\right)=\sum_{k=0}^{N-1} \prod_{j=0, j \neq k}^{N-1} \frac{N-j}{k-j}(N-k) \bar{\mu}_{b} \mathrm{e}^{-(N-k) \bar{\mu}_{b} t_{D}} .
$$

In order to derive the probability of $T_{D}$ exceeding a given threshold $D_{t h}$, we integrate the above pdf $f_{T_{D} \mid q=0}\left(t_{D}\right)$ over the region $\left[D_{t h}, \infty\right)$, which is expressed as

$$
\begin{aligned}
\operatorname{Pr}\left(T_{D}>D_{t h} \mid q=0\right) & =\int_{D_{t h}}^{\infty} f_{T_{D} \mid q=0}\left(t_{D}\right) d t_{D} \\
& =\sum_{k=0}^{N-1} \prod_{j=0, j \neq k}^{N-1} \frac{N-j}{k-j} \mathrm{e}^{-(N-k) \bar{\mu}_{b} D_{t h}} .
\end{aligned}
$$

2) Individual User-Delay: When the FA is $q=0$, the individual user-delay is solely determined by the quality of the wireless link connecting the $\mathrm{MU} \mathcal{A}$ to the BS. As a result, according to Lemma 2 , the individual user-delay $T_{\mathcal{A}}$ obeys an exponential distribution having a mean of $1 / \bar{\mu}_{b}$. Furthermore, the probability of $T_{\mathcal{A}}$ exceeding a given threshold $D_{t h}$ is derived as $\operatorname{Pr}\left(T_{\mathcal{A}}>D_{t h} \mid q=0\right)=\exp \left(-\bar{\mu}_{b} D_{t h}\right)$.

\section{B. Case 2: Fully Altruistic Behaviours $(q=1)$}

In this optimistic scenario, all the MUs in the target social group are completely altruistic. Since there are always some POs willing to forward the packet during the dissemination process, the BS is not invoked for multicasting the packet any more, once some of the MUs have initially received it from the BS.

1) Dissemination Delay: When the FA is $q=1$, by substituting $n_{k}=k$ into Eqs.(10) (12) in Section III-B, we know that the state transition delays $\left\{T_{k}, k=1, \ldots,(N-567\right.$ 1) $\}$ are independent exponentially distributed variables associ- 568 ated with the rates of $\left\{\tilde{\mu}_{k}=k(N-k) \bar{\mu}_{s}, k=1,2, \ldots,(N-569\right.$ 1)\}. Furthermore, by substituting $k=0$ into Eqs.(13) (15) in 570 Section III-B, the initial state transition delay $T_{0}$ is also an expo- 571 nentially distributed variable associated with a rate of $\tilde{\mu}_{0}=572$ $N \bar{\mu}_{b}$. Note furthermore that $T_{0}$ is also independent of $\left\{T_{k}, k=573\right.$ $1, \ldots,(N-1)\}$. Since the dissemination delay is defined as 574 $T_{D}=\sum_{k=0}^{N-1} T_{k}, T_{D}$ obeys the hypoexponential distribution. 575

However, the rates of $\left\{\tilde{\mu}_{k}=k(N-k) \bar{\mu}_{s}, k=576\right.$ $1,2, \ldots,(N-1)\}$ associated with $\left\{T_{k}, k=1, \ldots,(N-1)\right\} 577$ exhibit a symmetric structure. For example, the rates of $T_{k} 578$ and $T_{N-k}$ share the same value of $k(N-k) \bar{\mu}_{s}$. Hence, the 579 closed-form equation for the tdf of $T_{D}$ may only be expressed 580 in the form of a continuous phase-type distribution [35]. As a 581 result, when $q=1$, the transition rate matrix of the PBMC is 582 expressed as

$$
\mathbf{P}=\left(\begin{array}{cccccc}
-\tilde{\mu}_{0} & \tilde{\mu}_{0} & 0 & \cdots & 0 & 0 \\
0 & -\tilde{\mu}_{1} & \tilde{\mu}_{1} & \ddots & 0 & 0 \\
\vdots & \ddots & -\tilde{\mu}_{k} & \tilde{\mu}_{k} & \ddots & \vdots \\
0 & 0 & \ddots & -\tilde{\mu}_{N-2} & \tilde{\mu}_{N-2} & 0 \\
0 & 0 & \ldots & 0 & -\tilde{\mu}_{N-1} & \tilde{\mu}_{N-1} \\
0 & 0 & \ldots & 0 & 0 & 0
\end{array}\right)=\left(\begin{array}{cc}
\mathbf{Q} & \mathbf{Q}_{0} \\
\mathbf{0} & 0
\end{array}\right),
$$

where $\mathbf{Q}$ is a $(N \times N)$-element matrix containing all the tran- 584 sition rates between transient states, $\mathbf{Q}_{0}$ is a $(N \times 1)$ column 585 vector containing all the transition rates from transient states to 586 the absorbing state $N$, whose last entry is $\widetilde{\mu}_{N-1}$ and finally, 587 the remaining entries are all zeros. As shown in Fig.3, the 588 packet dissemination process starts from the initial state 0. 589 Thus, the probability of $T_{D}$ exceeding a given threshold $D_{t h} 590$ is expressed as

$$
\operatorname{Pr}\left(T_{D} \geq D_{t h} \mid q=1\right)=\boldsymbol{\tau}_{1}^{T} \times \exp \left(D_{t h} \mathbf{Q}\right) \times \mathbf{1}_{N} .
$$

Note that in (28), the $(N \times 1)$ column vector $\boldsymbol{\tau}_{k+1}(0 \leq k \leq 592$ $N-1)$, whose $(k+1)$ th entry is one but all the others are 593 zeros, indicates that the PBMC starts at state $k$, while the 594 $(N \times 1)$ column vector $\mathbf{1}_{k+1}$, whose first $(k+1)$ entries are 595 ones and the remaining entries are zeros, indicates that the 596 PBMC process is absorbed at state $(k+1)$. The proof of $(28) 597$ can be found in [36].

2) Individual User-Delay: Given an event that the MU $\mathcal{A} 599$ successfully receives the packet at state $(k+1)(0 \leq k \leq N-600$ $1)$, the PBMC used for modelling the packet dissemination in 601 Fig. 3 is considered to be terminated at state $(k+1)$. According 602 to the physical meaning of both $\boldsymbol{\tau}_{k+1}$ and $\mathbf{1}_{k+1}$, similar to (28), 603 the probability of $T_{\mathcal{A}}$ exceeding the threshold $D_{t h}$, given that $\mathcal{A} 604$ receives the desired packet at state $(k+1)$ for $(0 \leq k \leq N-605$ 1), is expressed as

$$
\operatorname{Pr}\left(T_{\mathcal{A}} \geq D_{t h} \mid q=1, k+1\right)=\boldsymbol{\tau}_{1}^{T} \times \exp \left(D_{t h} \mathbf{Q}\right) \times \mathbf{1}_{k+1} .
$$

Since we have already derived the probability of $p_{k+1}=1 / N 607$ that $\mathcal{A}$ receives the packet at state $(k+1)$ in $(21)$, according to 608 the Bayesian principle [31], the probability of $T_{\mathcal{A}}$ exceeding the 609 


$$
\begin{aligned}
& \operatorname{Pr}\left(T_{\mathcal{A}} \geq D_{t h} \mid q=1\right)=\sum_{k=0}^{N-1} \operatorname{Pr}\left(T_{\mathcal{A}} \geq D_{t h} \mid q=1, k+1\right) \cdot p_{k+1} \\
& =\sum_{k=0}^{N-1} \frac{\boldsymbol{\tau}_{1}^{T} \times \exp \left(D_{t h} \mathbf{Q}\right) \times \mathbf{1}_{k+1}}{N}=\frac{\boldsymbol{\tau}_{1}^{T} \times \exp \left(D_{t h} \mathbf{Q}\right)}{N} \times \sum_{k=0}^{N-1} \mathbf{1}_{k+1} \\
& =\frac{\boldsymbol{\tau}_{1}^{T} \times \exp \left(D_{t h} \mathbf{Q}\right) \times \eta}{N}
\end{aligned}
$$

where $\eta=(N, N-1, \ldots, 1)^{T}$ is a $(N \times 1)$ column vector.

\section{Case 3: Moderately Altruistic Behaviours $(q=0.5)$}

Unfortunately, we are unable to derive the exact tdf for the scenario, when the FA is set to $q=0.5$. However, we are still able to offer some interesting insights concerning the delay metrics of this specific case. Substituting $q=0.5$ into the second term of (17), the average duration of the social group multicast process during the transition from state $k$ to $(k+1)$ for $k \geq 1$ can be given by

$$
\mathcal{E}\left[T_{k, s} \mid q=0.5\right]=\frac{1}{2^{k} \cdot \mu_{s, k}} \sum_{n_{k}=1}^{k}\left(\begin{array}{c}
k \\
n_{k}
\end{array}\right) \frac{1}{n_{k}} .
$$

According to Eq.(68.1) of [33], we arrive at the following lower bound for $\mathcal{E}\left[T_{k, s} \mid q=0.5\right]$, which is expressed as:

$$
\begin{aligned}
\mathcal{E}\left[T_{k, s} \mid q=0.5\right] & >\frac{1}{2^{k} \cdot \mu_{s, k}}\left[\sum_{n_{k}=0}^{k}\left(\begin{array}{c}
k \\
n_{k}
\end{array}\right) \frac{1}{n_{k}+1}-1\right] \\
& =\frac{1}{2^{k} \cdot \mu_{s, k}} \frac{2^{k+1}-k+2}{k+1} .
\end{aligned}
$$

Similarly, substituting $q=1.0$ into the second term of (17), the corresponding formula of $\mathcal{E}\left[T_{k, s} \mid q=1.0\right]$ for this fully altruistic behaviour may be expressed as $\mathcal{E}\left[T_{k, s} \mid q=1.0\right]=$ $1 /\left(k \mu_{s, k}\right)$. As a result, the ratio $\mathcal{R}_{k, s}$ of these two expressions can be formulated as

$$
\mathcal{R}_{k, s}=\frac{\mathcal{E}\left[T_{k, s} \mid q=0.5\right]}{\mathcal{E}\left[T_{k, s} \mid q=1.0\right]}>\frac{\left(2^{k+1}-k+2\right) k}{2^{k}(k+1)} .
$$

In the ideal scenario, when $k$ tends to infinity, this ratio can be expressed as $\lim _{k \rightarrow \infty} R_{k, s}>2$. Since the lower bound derived in (32) is very tight ${ }^{8}$, we can summarise that by assuming moderately altruistic behaviours, the average duration of the social group multicasting during the transition from state $k$ to $(k+1)$ is twice that of the fully altruistic scenario, provided that $k$ is sufficiently high.

Let us now demonstrate the tightness of the lower bound (32) in terms of the average dissemination delay. Substituting (32) into (19), the lower bound of the average dissemination delay

\footnotetext{
${ }^{8}$ The tightness of this lower bound will be demonstrated in the following paragraph in terms of the average dissemination delay.
}

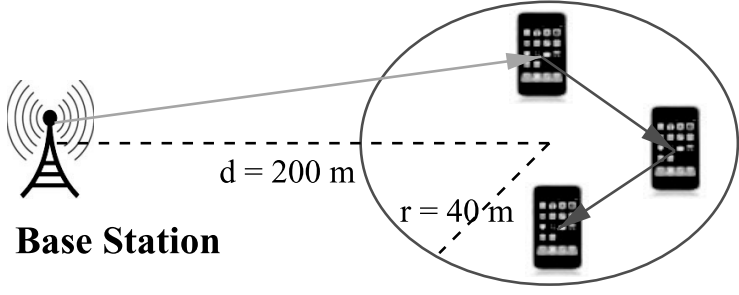

The Target MSN Group

Fig. 5. Geographic features for obtaining numerical results.

$\mathcal{E}\left[T_{D} \mid q=0.5\right]$ can be formulated as

$$
\begin{aligned}
\mathcal{E}\left[T_{D} \mid q=0.5\right] & =\sum_{k=0}^{N-1} \frac{1}{2^{k} \mu_{b, k}}+\sum_{k=1}^{N-1} \frac{1}{2^{k} \mu_{s, k}} \sum_{n_{k}=1}^{k}\left(\begin{array}{c}
k \\
n_{k}
\end{array}\right) \frac{1}{n_{k}} \\
& >\sum_{k=0}^{N-1} \frac{1}{2^{k} \mu_{b, k}}+\sum_{k=1}^{N-1} \frac{1}{2^{k} \cdot \mu_{s, k}} \frac{2^{k+1}-k+2}{k+1} .
\end{aligned}
$$

When we compute the exact result of $\mathcal{E}\left[T_{D} \mid q=0.5\right]$, which is 638 represented by the first line of (34), and its lower bound, which 639 is quantified by the second line of (34), then for a large social 640 group size $N$, such as $N=50 \sim 200$, using a set of other related 641 parameters in line with those of Fig. 6, the root-mean-square- 642 deviation (RMSD) of these two sets of results can be shown 643 to be 0.094 TS. Hence, we can claim that for a large social 644 group size, which represents our densely populated scenario, 645 the lower bound expressed in (34) can be regarded as an approx- 646 imate result of $\mathcal{E}\left[T_{D} \mid q=0.5\right]$. Furthermore, the tightness of the 647 lower bound derived in (33) can also be readily demonstrated. 648

Similarly, with the aid of (32), we can also obtain the lower 649 bound for the average individual user-delay $\mathcal{E}\left(T_{\mathcal{A}} \mid q=0.5\right)$.

\section{NUMERicAl RESUlts}

The parameters of the PHY layer are presented in TABLE II. 652 The specific parameters used for transmissions from the BS 653 to the MUs are in line with FDD-LTE standard ${ }^{9}$, while the 654 transmission parameters between the MUs are in line with the 655 commonly used 802.11 protocol [18].

As shown in Fig. 5, we assume that all MUs in the target 657 social group roam in a circular area having a radius of $r=40658$ $\mathrm{m}$ by obeying the uniform mobility model. The BS is $d=200659$ $\mathrm{m}$ away from the centre of the circular area. In this scenario, 660 the pdf $f_{Y_{s}}\left(y_{s}\right)$ of the distance between a pair of MUs is given 661 by Eq. (23) of [38], and $f_{Y_{b}}\left(y_{b}\right)$ between the BS and a MU can 662 be found in our technical report [39]. Substituting $f_{Y_{s}}\left(y_{s}\right)$ and 663 $f_{Y_{b}}\left(y_{b}\right)$ into (6), alongside the parameters offered in TABLE II, 664 we may obtain the average SPRP $\bar{\mu}_{s}$ and $\bar{\mu}_{b}$, which further lead 665 us to the analytical (ana) results for the various metrics. If we 666 let $q=0$ in our model, the corresponding analytical results are 667 derived for conventional BS-aided multicast. $\quad 668$

In order to obtain a reliable statistical characterization of the 669 simulation performance (sim), we repeatedly run Monte-Carlo 670

\footnotetext{
${ }^{9} \mathrm{We}$ assume a $1.8 \mathrm{GHz}$ carrier frequency in line with the LTE networks operated by the British company EE [37].
} 


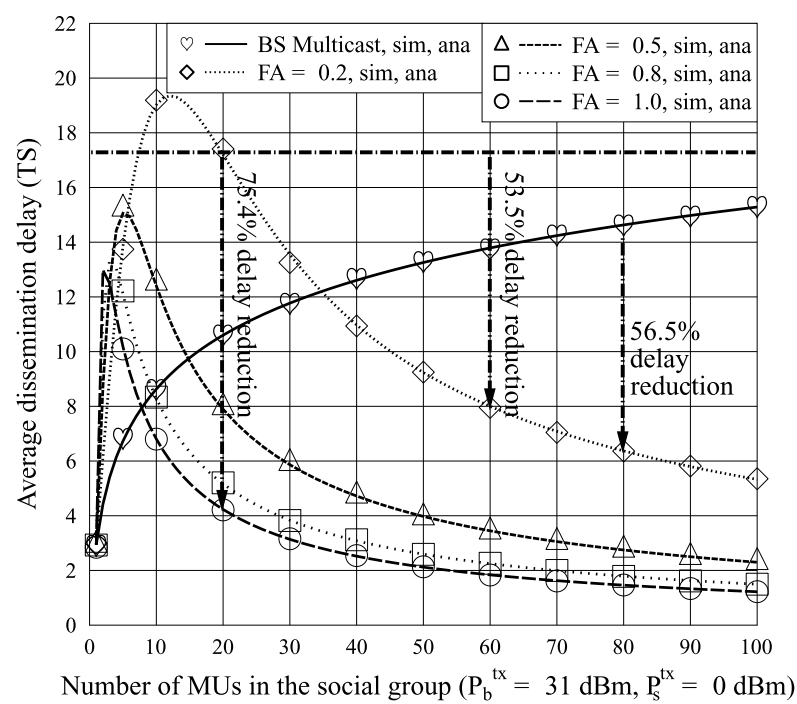

Fig. 6. Average dissemination delay affected by the number of MUs in the target social group, which is parameterized by the FA. The analytical results were evaluated from Eq. (19).

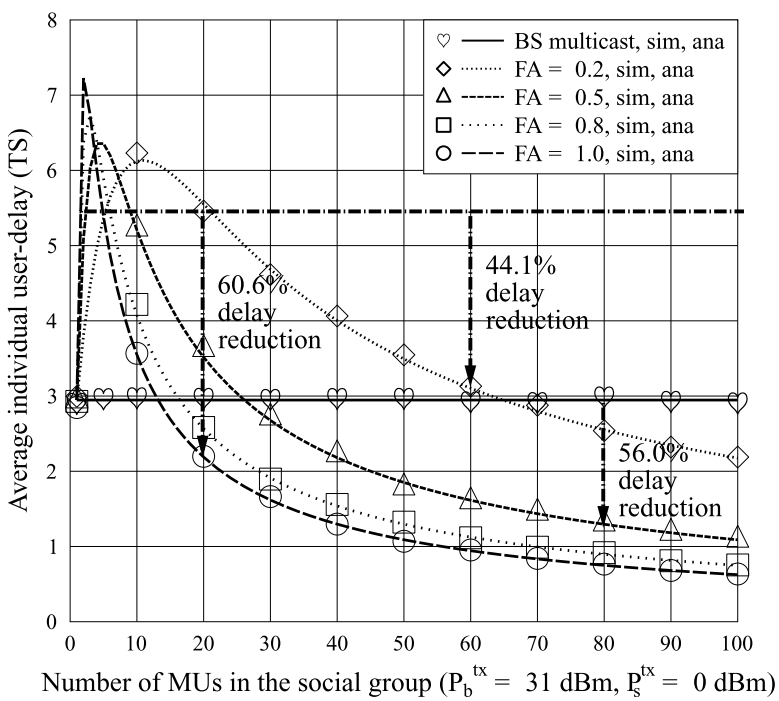

Fig. 7. Average individual user-delay as a function of the number of MUs in the target social group, which is parameterized by the FA. The analytical results were evaluated from (23).

simulations 10000 times and set the time-interval of our system to be $\Delta t=0.001 \mathrm{TS}$, where a TS can be considered as a packet duration. All the delay related metrics are evaluated by the number of TSs. In the numerical results of Figs. 6-8, we study the impact of the social group size $N$ on the delay metrics of the packet dissemination process without considering any specific content popularity.

\section{A. Delay Metrics for Uniform Mobility Model}

As shown in Fig. 6, when FA $\neq 0$, the average dissemination delay firstly increases, as the number of MUs is increased. When only a few MUs are in the target social group, a longer period is required for disseminating the packet to all of the group members due to the increasing content demand of the unserved MUs. However, by further increasing the number of

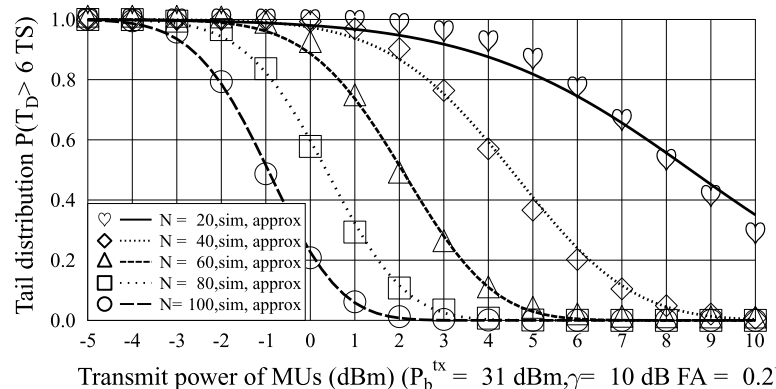

(a) Dissemination delay

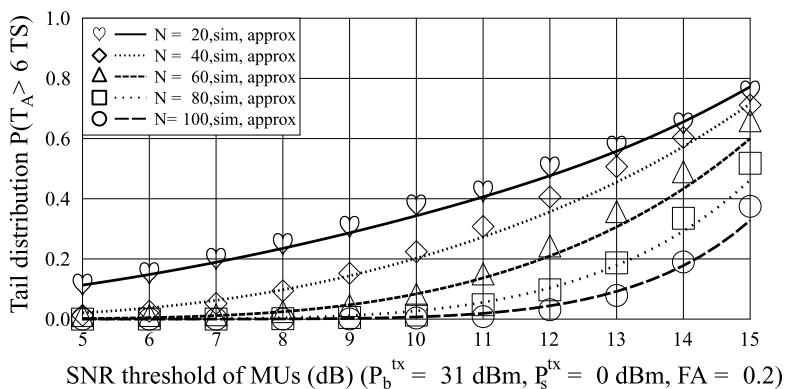

(b) Individual user-delay

Fig. 8. The tail distribution of the delay versus (a) the transmit power and (b) the SNR threshold for successful reception, which is parameterized by the number of MUs in the target social group. The analytical results were either directly or indirectly derived from Eq.(20).

MUs, the diversity gain incurred by the cooperation of the 685 multiple multicasters becomes sufficiently high to mitigate the 686 adverse effect of the increasing content demand. As a result, we 687 observe that the average dissemination delay decays after reach- 688 ing its peak, as the number of MUs is further increased. For 689 example, for $\mathrm{FA}=0.2$, the delay is reduced by $53.5 \%$, as the 690 number of MUs is increased from $N=20$ to 60. Furthermore, 691 a higher FA incurs a lower delay, since more POs are willing to 692 forward the packet after they successfully receive it. For exam- 693 ple, for $N=20$, the average dissemination delay is reduced 694 by $75.4 \%$, as the FA is increased from 0.2 to 1 . By contrast, 695 when $\mathrm{FA}=0$, the conventional BS-aided multicast technique 696 is invoked. However, as the number of the MUs increases, the 697 average dissemination delay also increases. We observe from 698 Fig. 6 that our approach is capable of reducing the average 699 dissemination delay of the conventional BS-aided multicast by 700 $56.5 \%$ for $N=80$, when a small FA value of 0.2 is assumed. 701

As shown in Fig. 7, when only a few MUs are in the tar- 702 get social group and the FA is non-zero, due to the users' 703 selfishness, fewer than two POs are willing to forward the 704 packet during the dissemination process. Therefore, we observe 705 from Fig. 7 that the average individual user-delay initially 706 increases, because it does not benefit from any diversity gain. 707 However, as we further increase the number of MUs, an increas- 708 ing number of POs become willing to forward the packet, 709 which substantially reduces the average individual user-delay, 710 as observed from Fig. 7. For example, for FA $=0.2$, the aver- 711 age individual user-delay is reduced by $44.1 \%$, as the number of 712 MUs is increased from $N=20$ to 60 . Nevertheless, when the 713 conventional BS-aided multicast is invoked, the average indi- 714 vidual user-delay, which only relies on the link connecting this 715 


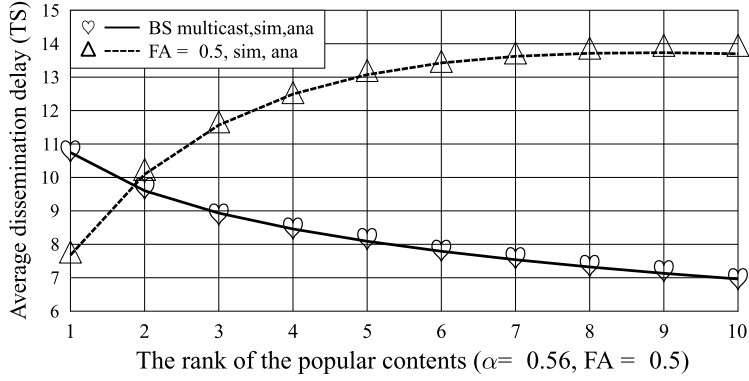

Fig. 9. Average dissemination delay as a function of the rank of the popular content. The transmit power of the BS is $P_{b}^{t x}=31 \mathrm{dBm}$ and the transmit power of a MU is $P_{t}^{t x}=0 \mathrm{dBm} . \mathcal{N}=100$ MUs independently request $\mathcal{M}=10$ ranked-popularity pieces of contents according to the request probabilities listed in TABLE I when $\alpha=0.56$. The analytical results were evaluated from Eq.(3).

specific MU to the BS, remains near-constant at 2.95 TS, as the number of MUs increases. Furthermore, the average individual user-delay is improved, when we increase the value of the FA. For example, given $N=20 \mathrm{MUs}$ in the target social group, the average individual user-delay is reduced by $60.6 \%$, as the FA is increased from 0.2 to 1.0. Additionally, given $N=80 \mathrm{MUs}$ in the target social group, the average individual user-delay drops from 2.95 TS to 1.3 TS, comparing the conventional BS-aided multicast to our approach associated with $\mathrm{FA}=0.5$.

Observe in Fig. 8(a) that the probability of the dissemination delay exceeding a threshold of $D_{t h}=6 \mathrm{TS}$ reduces upon increasing the transmit power of each MU. By contrast, as portrayed in Fig. 8(b), the probability of the individual userdelay exceeding the same threshold increases upon increasing the SNR threshold to be exceeded for ensuring successful packet reception. Our Gamma-distribution-based approximations match the simulation results.

Then, we study the average dissemination delay as a function of the specific popularity of the pieces of contents in Fig. 9. Observe from Fig. 9 that as a piece of contents becomes less popular, the average dissemination delay of our scheme increases, when we have a moderate degree of altruism associated with $\mathrm{FA}=0.5$. When a piece of content is less popular, fewer MUs may request this content, hence the resultant smaller social group fails to provide sufficient cooperative multicast opportunities for rapidly disseminating the packet across the social group. By contrast, since a less popular piece of contents results in a lower content demand, the average dissemination delay of the BS-aided multicast reduces, as the content becomes less popular. Furthermore, as shown in Fig. 9, our scheme associated with $\mathrm{FA}=0.5$ outperforms the conventional BS-aided multicast in terms of its delay of disseminating the most popular content. Nevertheless, the BS-adied multicast is more suitable for disseminating the less popular pieces of contents.

\section{B. Investigations Using Real Mobility Traces}

Let us now study the content dissemination performance in a densely-populated subway station scenario [40]. The mobility traces for this scenario can be downloaded from the CRAWDAD database ${ }^{10}$. The active area in this scenario is

\footnotetext{
${ }^{10}$ http://crawdad.cs.dartmouth.edu/kth/walkers/
}

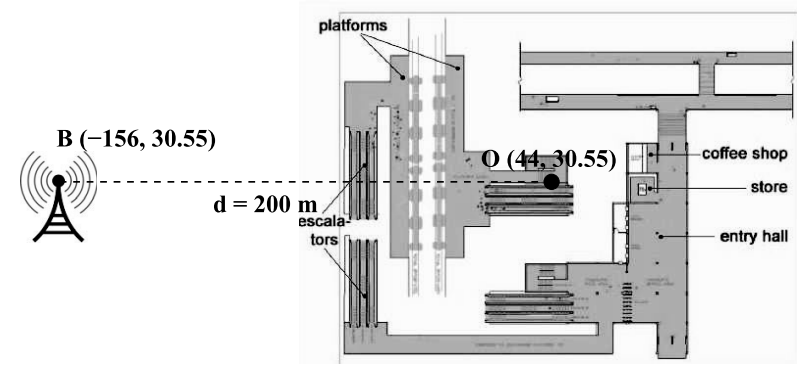

Fig. 10. A densely popluated subway station.

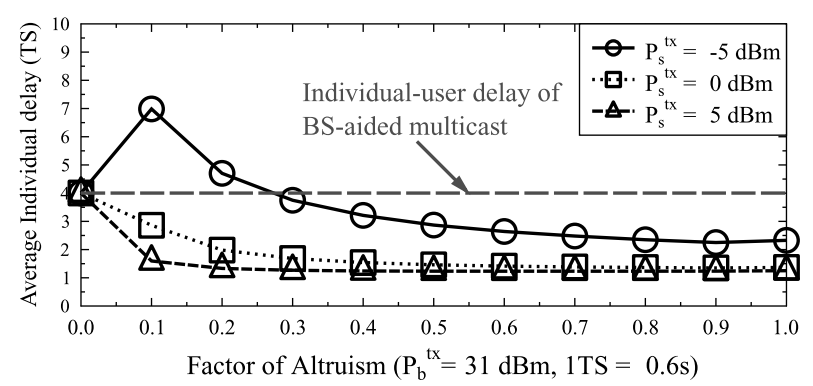

Fig. 11. Average individual user-delay in a subway station when all the MUs in the subway station form a grand social group for downloading a content of common interest.

$1921 \mathrm{~m}^{2}$. After analysing the mobility traces, the centre $O$ of 755 the active area is found to be at the coordinates of $(44,30.55) 756$ $\mathrm{m}$, as shown in Fig. 10. In our simulations, we placed the BS at 757 the point $(-156,30.55) \mathrm{m}$, which is $200 \mathrm{~m}$ away from the cen- 758 tre of the subway station. Since the MUs arrive/depart either 759 through the entrances or during the arrival/departure of trains, 760 the number of MUs is dynamic during the simulation time. As 761 a result, we cannot readily obtain the dissemination delay in 762 this scenario. However, we are still able to evaluate the indi- 763 vidual user-delay, when our content dissemination scheme and 764 conventional BS-aided multicast scheme are invoked. Again, 765 the physical layer parameters are summarised in TABLE II. 766 Since the positions of the MUs are captured every $0.6 \mathrm{~s}$ in this 767 mobility trace, in our simulations we set the basic time inter- 768 val of $\Delta t=0.6 \mathrm{~s}$ as a single TS, which can be considered as a 769 packet's duration. Then the delay was evaluated in terms of the 770 number of TSs.

We first assume that all the MUs in the subway station form 772 a large social group in order to download the train schedule of 773 common interest. Observe from Fig. 11 that for the cases of 774 $P_{S}^{t x}=0 \mathrm{dBm}$ and $P_{S}^{t x}=5 \mathrm{dBm}$, the average individual user- 775 delay is reduced, as we increase the FA from 0.0 to 1.0. For 776 $P_{s}^{t x}=-5 \mathrm{dBm}$, when $\mathrm{FA}$ is increased from 0.0 to 0.1 , we 777 observe an increasing average individual user-delay. This is 778 because the SPRP between the MUs is low and also, because 779 fewer POs are willing to forward the packet. As FA becomes 780 higher, more POs may join to assist the packet dissemina- 781 tion process, which significantly reduces the average individual 782 user-delay. Specifically, when FA $=0$, conventional BS-aided 783 multicast is invoked for disseminating the packets. For $P_{s}^{t x}=0 \quad 784$ or $5 \mathrm{dBm}$, if the MUs become only modestly altruistic, say we 785 have $\mathrm{FA}=0.1$, our content dissemination scheme outperforms 786 


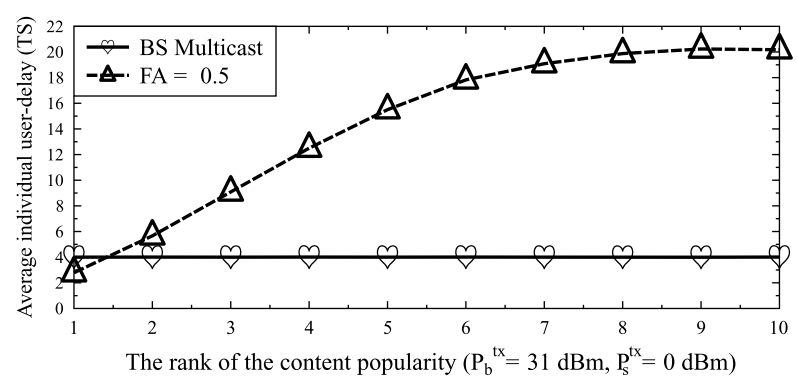

Fig. 12. Average individual user-delay in a subway station when the MUs in the subway station independently request $\mathcal{M}=10$ ranked-popularity pieces of contents according to the probabilities listed in TABLE I when $\alpha=1$.

A TS, during which the successful packet

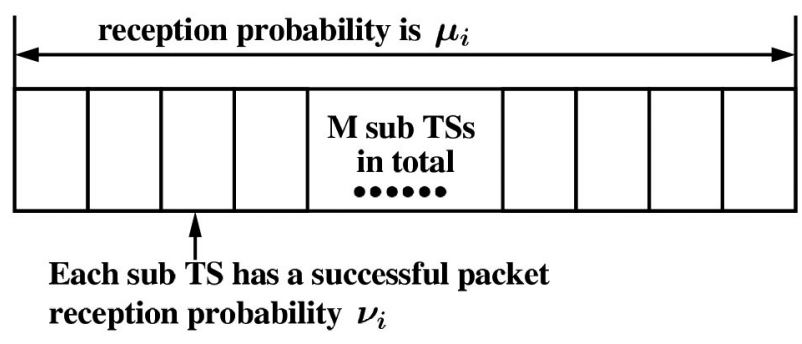

Fig. 13. The structure of a TS.

the conventional BS-aided multicast. For $P_{s}^{t x}=-5 \mathrm{dBm}$, our scheme starts to outperform the classic BS-aided multicast, provided that FA is higher than 0.4 .

We then study the impact of the specific content popularity on the average individual user-delay in a subway station. Observe from Fig. 12 that when disseminating the most popular content in the subway station, our dissemination scheme associated with $\mathrm{FA}=0.5$ outperforms the conventional BS-aided multicast. However, the BS-aided multicast is more suitable for disseminating less popular content in this scenario. The reason behind this trend is the same as that associated with Fig. 9.

\section{Vi. Conclusions}

In this paper, we proposed a social group multicast aided content dissemination scheme as a supplement to the conventional cellular system. The content popularity is modelled by a Zipf distribution and the concept of FA was introduced for the sake of quantifying the probability of a PO forwarding a packet of the content of common interest. In our scheme, the BSs are invoked for multicasting the packet at the initial stage, as well as when no POs are willing to share the packet with others. By modelling the packet dissemination process as a PBMC, closed-form expressions were derived for the statistical properties of the various delay metrics. We demonstrated that our approach outperforms the conventional BS-adied multicast in terms of both the dissemination delay and the individual-user delay, especially when the density of MUs in a target group is high. Furthermore, we found that our approach is more suitable for disseminating a more popular content. By contrast, the conventional BS-aided multicast performs better for disseminating a less popular content.
APPENDIX A

As shown in Fig. 13, a TS is divided into $M$ sub-TSs, each 819 of which has a duration of $\Delta t=1 / M$ TS. We assume that the 820 SPRP in a sub-TS is $\nu_{i}$. As a result, given the SPRP $\bar{\mu}_{i}$ in a 821 TS, we may derive the relation between $\bar{\mu}_{i}$ and $\nu_{i}$, which is 822 expressed as

$$
\bar{\mu}_{i}=\sum_{j=1}^{M}\left(1-v_{i}\right)^{j-1} v_{i}=1-\left(1-v_{i}\right)^{M} .
$$

Rewriting the above expression, we obtain

$$
v_{i}=1-\left(1-\bar{\mu}_{i}\right)^{1 / M}=1-\left(1-\bar{\mu}_{i}\right)^{\Delta t},
$$

where the second equality is derived according to $\Delta t=1 / M 825$ TS. If we expand $\left(1-\bar{\mu}_{i}\right)^{\Delta t}$ according to the Taylor series, we 826 have

$$
\left(1-\bar{\mu}_{i}\right)^{\Delta t}=\sum_{n=0}^{\infty}\left(\begin{array}{c}
\Delta t \\
n
\end{array}\right)\left(-\bar{\mu}_{i}\right)^{n}=1-\bar{\mu}_{i} \Delta t+O\left(\bar{\mu}_{i}^{2}\right),
$$

where $O\left(\bar{\mu}_{i}^{2}\right)$ is the infinitesimal by small quantity on the same 828 order as $\bar{\mu}_{i}^{2}$. Substituting the above equation into (36), we have 829

$$
v_{i}=\bar{\mu}_{i} \Delta t+O\left(\bar{\mu}_{i}^{2}\right) \approx \bar{\mu}_{i} \Delta t .
$$

According to our experiments, if we vary $\bar{\mu}_{i}$ from 0 to $0.8,830$ the root-mean-square-deviation (RMSD) between the exact $v_{i} 831$ given by (36) and the approximated $v_{i}$ given by $(38)$ is $9.45 \times 832$ $10^{-4}$. As a result, it is reasonable to claim that $\nu_{i} \approx \bar{\mu}_{i} \Delta t$.

APPENDIX B

THE PROOF OF LEMMA 2

During a time interval $\Delta t$, the PBMC may transit from state 836 $k$ to $(k+1)$ with a probability of $\tilde{\mu}_{k} \Delta t$. Naturally, the success- 837 ful state transition first occurring during the $\left(M_{k}=m_{k}\right)$-th $\Delta t \quad 838$ interval obeys a geometric distribution. According to the PMF 839 of a geometric distribution having a parameter of $\tilde{\mu}_{k} \Delta t$, we 840 arrive at:

$$
\begin{gathered}
\operatorname{Pr}\left(M_{k} \Delta t \leq m_{k} \Delta t\right)=\sum_{m=1}^{m_{k}}\left(1-\tilde{\mu}_{k} \Delta t\right)^{m-1} \tilde{\mu}_{k} \Delta t \\
\operatorname{Pr}\left(M_{k} \Delta t \leq\left(m_{k}+1\right) \Delta t\right)=\sum_{m=1}^{m_{k}+1}\left(1-\tilde{\mu}_{k} \Delta t\right)^{m-1} \tilde{\mu}_{k} \Delta t
\end{gathered}
$$

The continuous-valued delay of the adjacent-state transition is 842 denoted as $T_{k}=M_{k} \Delta t$, which is associated with a specific 843 value of $t_{k}=m_{k} \Delta t$. Hence, we may derive the pdf of $T_{k}$ as: 844

$$
\begin{aligned}
f_{T_{k}}\left(t_{k}\right) & =\lim _{\Delta t \rightarrow 0} \frac{\operatorname{Pr}\left(T_{k} \leq t_{k}+\Delta t\right)-\operatorname{Pr}\left(T_{k} \leq t_{k}\right)}{\Delta t} \\
& =\lim _{\Delta t \rightarrow 0} \frac{\operatorname{Pr}\left(M_{k} \Delta t \leq\left(m_{k}+1\right) \Delta t\right)-\operatorname{Pr}\left(M_{k} \Delta t \leq m_{k} \Delta t\right)}{\Delta t} \\
& =\lim _{\Delta t \rightarrow 0} \frac{\left(1-\tilde{\mu}_{k} \Delta t\right)^{m_{k}} \tilde{\mu}_{k} \Delta t}{\Delta t}=\lim _{\Delta t \rightarrow 0} \tilde{\mu}_{k} \mathrm{e}^{-m_{k} \tilde{\mu}_{k} \Delta t} \\
& =\tilde{\mu}_{k} \cdot \mathrm{e}^{-\tilde{\mu}_{k} t_{k}},
\end{aligned}
$$


845 where the last two lines are derived based on $\lim _{\Delta t \rightarrow 0} \tilde{\mu}_{k} \Delta t=$ $1-\mathrm{e}^{-\widetilde{\mu}_{k} \Delta t}$ and $m_{k}=t_{k} / \Delta t$, respectively.

\section{REFERENCES}

[1] N. Kayastha, D. Niyato, P. Wang, and E. Hossain, "Applications, architectures, and protocol design issues for mobile social networks: A survey," Proc. IEEE, vol. 99, no. 12, pp. 2130-2158, Dec. 2011.

[2] A. Vahdat and D. Becker, "Epidemic routing for partially-connected ad hoc networks," Master thesis, Dept. Comput. Sci., Duke Univ., Durham, NC 27708 USA, Tech. Rep., 2000.

[3] Y.-K. Ip, W.-C. Lau, and O.-C. Yue, "Performance modeling of epidemic routing with heterogeneous node types," in Proc. IEEE Int. Conf. Commun. (ICCŠO8), May 2008, pp. 219-224.

[4] A. Clementi, F. Pasquale, and R. Silvestri, "Opportunistic manets: Mobility can make up for low transmission power," IEEE/ACM Trans. Netw., vol. 21, no. 2, pp. 610-620, Feb. 2013.

[5] H. Sun and C. Wu, "Epidemic forwarding in mobile social networks," in Proc. IEEE Int. Conf. Commun. (ICC'12), Jun. 2012, pp. 1421-1425.

[6] J. Whitbeck, V. Conan, and M. D. de Amorim, "Performance of opportunistic epidemic routing on edge-markovian dynamic graphs," IEEE Trans. Commun., vol. 59, no. 5, pp. 1259-1263, May 2011.

[7] S. Ioannidis, A. Chaintreau, and L. Massoulie, "Optimal and scalable distribution of content updates over a mobile social network," in Proc. IEEE INFOCOM, 2009, pp. 1422-1430.

[8] C. Boldrini, M. Conti, and A. Passarella, "Contentplace: Social-aware data dissemination in opportunistic networks," in Proc. 11th Int. Symp. Model. Anal. Simul. Wireless Mobile Syst. (MSWiM'08), 2008, pp. 203210.

[9] D. Niyato, P. Wang, W. Saad, and A. Hj申andrungnes, "Controlled coalitional games for cooperative mobile social networks," IEEE Trans. Veh. Technol., vol. 60, no. 4, pp. 1812-1824, May 2011.

[10] K. Akkarajitsakul, E. Hossain, and D. Niyato, "Cooperative packet delivery in hybrid wireless mobile networks: A coalitional game approach," IEEE Trans. Mobile Comput., vol. 12, no. 5, pp. 1-15, May 2013.

[11] B. Han, P. Hui, V. Kumar, M. Marathe, J. Shao, and A. Srinivasan, "Mobile data offloading through opportunistic communications and social participation," IEEE Trans. Mobile Comput., vol. 11, no. 5, pp. 821-834, May 2012.

[12] Y. Li, M. Qian, D. Jin, P. Hui, Z. Wang, and S. Chen, "Multiple mobile data offloading through disruption tolerant networks," IEEE Trans. Mobile Comput., vol. 13, no. 7, pp. 1579-1596, Jul. 2014.

[13] R. Groenevelt, P. Nain, and G. Koole, "The message delay in mobile ad hoc networks," Perform. Eval., vol. 62, nos. 1-4, pp. 210-228, Oct. 2005.

[14] T. Karagiannis, J.-Y. Le Boudec, and M. Vojnovic, "Power law and exponential decay of intercontact times between mobile devices," IEEE Trans. Mobile Comput., vol. 9, no. 10, pp. 1377-1390, Oct. 2010.

[15] J. Hu, L.-L. Yang, and L. Hanzo, "Mobile social networking aided content dissemination in heterogeneous networks," China Commun., vol. 10, no. 6, p. 1, 2013.

[16] J. Wang, S. Park, D. Love, and M. Zoltowski, "Throughput delay tradeoff for wireless multicast using hybrid-ARQ protocols," IEEE Trans. Commun., vol. 58, no. 9, pp. 2741-2751, Sep. 2010.

[17] J. Seo, T. Kwon, and V. Leung, "Social groupcasting algorithm for wireless cellular multicast services," IEEE Commun. Lett., vol. 17, no. 1, pp. 47-50, Jan. 2013.

[18] Information Technology-Telecommunications and Information Exchange Between Systems Local and Metropolitan Area NetworksSpecific Requirements Part 11: Wireless LAN Medium Access Control (MAC) and Physical Layer (PHY) Specifications, ISO/IEC/IEEE Standard 8802-11:2012(E), Nov. 2012, pp. 1-2798.

[19] Z. Gong and M. Haenggi, "Interference and outage in mobile random networks: Expectation, distribution, and correlation," IEEE Trans. Mobile Comput., vol. 13, no. 2, pp. 337-349, Feb. 2014.

[20] H. Kwon and B. G. Lee, "Cooperative power allocation for broadcast/ multicast services in cellular OFDM systems," IEEE Trans. Commun., vol. 57, no. 10, pp. 3092-3102, Oct. 2009.

[21] D. Feng, L. Lu, Y. Yuan-Wu, G. Li, G. Feng, and S. Li, "Device-to-device communications underlaying cellular networks," IEEE Trans. Commun., vol. 61, no. 8, pp. 3541-3551, Aug. 2013.

[22] R. E. Hattachi and J. Erfanian, "NGMN 5G White Paper version 1.0," Next Generation Mobile Networks (NGMN), NGMN Alliance, Frankfurt, Germany, 2015.
[23] M. Cha, H. Kwak, P. Rodriguez, Y.-Y. Ahn, and S. Moon, "I tube, you 916 tube, everybody tubes," in Proc. 7th ACM SIGCOMM Conf. Internet 917 Meas. (IMC'07), Oct. 2007, p. 1.

[24] M. Zink, K. Suh, Y. Gu, and J. Kurose, "Characteristics of YouTube 919 network traffic at a campus network-Measurements, models, and impli- 920 cations," Comput. Netw., vol. 53, no. 4, pp. 501-514, Mar. 2009.

[25] K. Shanmugam, N. Golrezaei, A. G. Dimakis, A. F. Molisch, and 922 G. Caire, "FemtoCaching: Wireless content delivery through distributed 923 caching helpers," IEEE Trans. Inf. Theory, vol. 59, no. 12, pp. 8402-8413, 924 Dec. 2013.

[26] M. Taghizadeh, K. Micinski, S. Biswas, C. Ofria, and E. Torng, 926 "Distributed cooperative caching in social wireless networks," IEEE 927 Trans. Mobile Comput., vol. 12, no. 6, pp. 1037-1053, Jun. 2013.

[27] T. Rappaport, Wireless Communications: Principles and Practice, 2nd ed. 929 Upper Saddle River, NJ, USA: Prentice-Hall, 2001.

[28] H. Zhang, Z. Zhang, and H. Dai, "Gossip-based information spreading 93 in mobile networks," IEEE Trans. Wireless Commun., vol. 12, no. 11, 932 pp. 5918-5928, Nov. 2013.

29] X. Wang, Q. Peng, and Y. Li, "Cooperation achieves optimal multi- 934 cast capacity-delay scaling in MANET," IEEE Trans. Commun., vol. 60, 935 no. 10, pp. 3023-3031, Oct. 2012.

[30] J. Hu, L.-L. Yang, and L. Hanzo, "Maximum average service rate and 937 optimal queue scheduling of delay-constrained hybrid cognitive radio in 938 Nakagami fading channels," IEEE Trans. Veh. Technol., vol. 62, no. 5, 939 pp. 2220-2229, Jun. 2013.

[31] P. V. Mieghem, Performance Analysis of Communications Networks and Systems. Cambridge, U.K.: Cambridge Univ. Press, 2005.

[32] M. Derakhshani and T. Le-Ngoc, "Aggregate interference and capacityoutage analysis in a cognitive radio network," IEEE Trans. Veh. Technol., vol. 61, no. 1, pp. 196-207, Jan. 2012.

[33] I. S. Gradshteyn and I. M. Ryzhik, Table of Integrals, Series, and 946 Products, 7th ed. New York, NY, USA: Elsevier/Academic, 2007.

[34] W. Gao, Q. Li, B. Zhao, and G. Cao, "Social-aware multicast in 948 disruption-tolerant networks," IEEE/ACM Trans. Netw., vol. 20, no. 5, 949 pp. 1553-1566, Oct. 2012.

[35] M. W. Fackrell, "Characterization of matrix-exponential distributions," 951 Ph.D. dissertation, Faculty of Eng., Comput. Math. Sci., School of Appl. 952 Math., Univ. Adelaide, Adelaide, South Australia, 5005, Australia, 2003. 953

[36] G. Latouche and V. Ramaswami, Introduction to Matrix Analytic Methods 954 in Stochastic Modeling. Philadelphia, PA, USA: SIAM, 1999.

[37] Office of Communications. "Notice of proposed variation of Everything 956 Everywhere's $1800 \mathrm{MHz}$ spectrum licences to allow use of LTE 957 and WiMAX technologies," Ofcom, Mar. 2012 [Online]. Available: 958 http://stakeholders.ofcom.org.uk/consultations/variation-1800mhz-lte- 959 wimax/summary.

[38] C. Bettstetter, H. Hartenstein, and X. Pérez-Costa, "Stochastic properties of the random waypoint mobility model," Wireless Netw., vol. 10, no. 5, pp. 555-567, 2004.

[39] J. Hu, L.-L. Yang, and L. Hanzo, "Stochastic geometry in the cellular networks," Univ. Southampton, SO17 1BJ, U.K., Tech. Rep., 2015 [Online]. Available: http://eprints.soton.ac.uk/id/eprint/374932.

[40] O. Helgason, S. T. Kouyoumdjieva, and G. Karlsson, "Opportunistic communication and human mobility," IEEE Trans. Mobile Comput., vol. 13, no. 7, pp. 1597-1610, Jul. 2014.

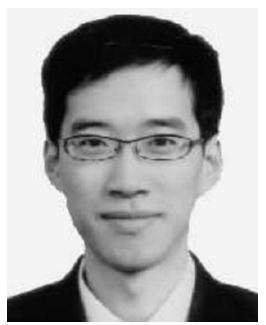

Jie Hu (S'11-M'16) received the B.Eng. degree 970 in communication engineering and the M.Eng. 971 degree in communication and information sys- 972 tem from the School of Communication and 973 Information Engineering, Beijing University of Posts 974 and Telecommunications, Beijing, China, in 2008975 and 2011, respectively, and the Ph.D. degree from 976 the Southampton Wireless Group, University of 977 Southampton, Southampton, U.K., in 2015.

Since March 2016, he has been working with 979 the School of Communication and Information 980 Engineering, University of Electronic Science and Technology of China 981 (UESTC), Chengdu, China, as a Lecturer. He has a broad range of interests 982 in wireless communication and networking, such as cognitive radio and cog- 983 nitive networks, queuing analysis, resource allocation and scheduling, ad hoc 984 wireless networks, and mobile social networks. 
986

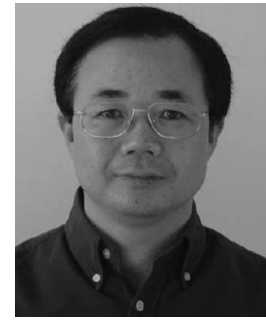

Lie-Liang Yang (M'98-SM'02-F'16) received the B.Eng. degree in communications engineering from Shanghai TieDao University, Shanghai, China, in 1988, and the M.Eng. and Ph.D. degrees in communications and electronics from Beijing (Northern) Jiaotong University, Beijing, China, in 1991 and 1997, respectively. From June 1997 to December 1997, he was a Visiting Scientist at the Institute of Radio Engineering and Electronics, Academy of Sciences of the Czech Republic, Prague, Czech Republic. Since December 1997, he has been with the University of Southampton, Southampton, U.K., where he is currently a Professor of Wireless Communications with the School of Electronics and Computer Science. His research interests include wireless communications, wireless networking, and signal processing for wireless communications. $\mathrm{He}$ has authored more than 300 research papers in journals and conference proceedings, authored or coauthored three books, and also published several book chapters.

Prof. Yang is a Fellow of The Institution of Engineering and Technology, U.K. He served as an Associate Editor for the IEEE TRANSACTIONS ON VEHICULAR TECHNOLOGY and the Journal of Communications and Networks, and is currently an Associate Editor of the IEEE ACCESS and the Security and Communication Networks Journal.

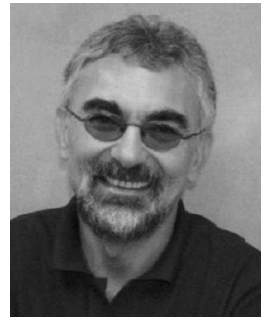

Lajos Hanzo (M'91-SM'92-F'03) received the 1009 degree in electronics, in 1976, the Doctorate degree, 1010 in 1983, and the D.Sc. degree. In 2009, he received 1011 the Honorary Doctorate "Doctor Honoris Causa" 1012 from the Technical University of Budapest, Budapest, 1013 Hungary. During his 35-year career in telecommuni- 1014 cations, he has held various research and academic 1015 posts in Hungary, Germany, and the U.K. Since 1016 1986, he has been with the School of Electronics 1017 and Computer Science, University of Southampton, 1018 Southampton, U.K., where he holds the Chair in 1019 telecommunications. He has successfully supervised 80 Ph.D. students, coau- 1020 thored 20 John Wiley/IEEE Press books on mobile radio communications 1021 totalling in excess of 10,000 pages, published over 1500 research entries at 1022 IEEE Xplore, acted both as TPC and General Chair of the IEEE conferences, 1023 presented keynote lectures and has been awarded a number of distinctions. 1024 Currently, he is directing a 60-strong academic research team, working on a 1025 range of research projects in the field of wireless multimedia communications 1026 sponsored by industry, the Engineering and Physical Sciences Research Council 1027 (EPSRC) U.K., the European IST Programme and the Mobile Virtual Centre of 1028 Excellence (VCE), U.K. He is an enthusiastic supporter of industrial and aca- 1029 demic liaison and he offers a range of industrial courses. He is also a Governor 1030 of the IEEE VTS. From 2008 to 2012, he was the Editor-in-Chief of the IEEE 1031 Press and a Chaired Professor also at Tsinghua University, Beijing, China. His 1032 research is funded by the European Research Council's Senior Research Fellow 1033 Grant. He is the Fellow of the REng, IET, and EURASIP. 